\title{
SCIENCE WITH ASKAP
}

\section{The Australian Square-Kilometre-Array Pathfinder}

\author{
S. Johnston* - R. Taylor - M. Bailes • \\ N. Bartel - C. Baugh - M. Bietenholz • \\ C. Blake - R. Braun - J. Brown - S. \\ Chatterjee - J. Darling - A. Deller . \\ R. Dodson - P. Edwards · R. Ekers • \\ S. Ellingsen - I. Feain - B. Gaensler . \\ M. Haverkorn · G. Hobbs · A. Hopkins · \\ C. Jackson - C. James · G. Joncas . \\ V. Kaspi · V. Kilborn - B. Koribalski · \\ R. Kothes - T. Landecker • A. Lenc . \\ J. Lovell - J.-P. Macquart - R. Manchester . \\ D. Matthews - N. McClure-Griffiths . \\ R. Norris · U.-L. Pen - C. Phillips • \\ C. Power - R. Protheroe - E. Sadler . \\ B. Schmidt • I. Stairs - L. Staveley-Smith . \\ J. Stil · S. Tingay • A. Tzioumis · \\ M. Walker · J. Wall ${ }^{\dagger}$. M. Wolleben \\ Received: date / Accepted: date
}

*Email: Simon.Johnston@csiro.au

†Overall Editor

S. Johnston · R. Braun · P. Edwards · R. Ekers · I. Feain · G. Hobbs · C. Jackson · B. Koribalski · R. Manchester · N. McClure-Griffiths · R. Norris · C. Phillips · A. Tzioumis

Australia Telescope National Facility, CSIRO, PO Box 76, Epping, NSW 1710, Australia

R. Taylor · J. Brown · R. Kothes · J. Stil

Dept of Physics and Astronomy, University of Calgary, Calgary, AB T2N 1N4, Canada

M. Bailes · C. Blake · A. Deller · V. Kilborn · A. Lenc · C. Power

Centre for Astrophysics and Supercomputing, Swinburne University of Technology, PO Box 218, Hawthorn, Vic 3122, Australia

N. Bartel · M. Bietenholz

Dept of Physics and Astronomy, York University, Toronto, ON M3J 1P3, Canada

C. Baugh

Institute for Computational Cosmology, University of Durham, Durham, DH1 3LE, UK

M. Bietenholz

Hartebeesthoek Radio Observatory, PO Box 443, Krugersdorp 1740, South Africa

S. Chatterjee · B. Gaensler · A. Hopkins · E. Sadler

School of Physics, The University of Sydney, NSW 2006, Australia

J. Darling

Center for Astrophysics and Space Astronomy, University of Colorado, 389 UCB, Boulder, CO 80309-0389, USA 
R. Dodson

Observatorio Astronomico Nacional, Alcara de Henares, Spain

S. Ellingsen · J. Lovell

School of Mathematics and Physics, University of Tasmania, Private Bag 21, Hobart, Tas 7001, Australia

M. Haverkorn

NRAO Jansky Fellow: Astronomy Dept, University of California-Berkeley, Berkeley, CA 94720, USA

C. James · R. Protheroe

School of Chemistry \& Physics, University of Adelaide, SA 5006, Australia

G. Joncas

Dept de Physique et Observatoire du Mont Megantic, Universite Laval, Quebec, QC G1K 7P4, Canada

V. Kaspi

Dept of Physics, McGill Unversity, Montreal, QC H3A 2T8, Canada

T. Landecker · M. Wolleben

Dominion Radio Astrophysical Observatory, Herzberg Institute of Astrophysics, NRC, Penticton, BC, Canada

J.-P. Macquart

NRAO Jansky Fellow: Astronomy Dept, California Institute of Technology, Pasadena, CA 91125, USA

D. Matthews

Dept of Physics, La Trobe University, Vic 3086, Australia

U.-L. Pen

Canadian Insititute for Theoretical Astrophysics, University of Toronto, Toronto, ON M5S 3H8, Canada

B. Schmidt

Mount Stromlo and Siding Spring Observatory, Private Bag, Weston Creek, Canberra, ACT 2601, Australia

I. Stairs · J. Wall

Dept of Physics and Astronomy, University of British Columbia, 6224 Agricultural Road, Vancouver, BC V6T 1Z1, Canada

L. Staveley-Smith

School of Physics, University of Western Australia, Crawley, WA 6009, Australia

S. Tingay

Dept of Imaging and Applied Physics, Curtin University of Technology, Bentley, WA, Australia

M. Walker

Manly Astrophysics Workshop Pty Ltd, Manly, NSW 2095, Australia 


\begin{abstract}
The future of $\mathrm{cm}$ and m-wave astronomy lies with the Square Kilometre Array (SKA), a telescope under development by a consortium of 17 countries. The SKA will be 50 times more sensitive than any existing radio facility. A majority of the key science for the SKA will be addressed through large-area imaging of the Universe at frequencies from $300 \mathrm{MHz}$ to a few $\mathrm{GHz}$.
\end{abstract}

The Australian SKA Pathfinder (ASKAP) is aimed squarely in this frequency range, and achieves instantaneous wide-area imaging through the development and deployment of phase-array feed systems on parabolic reflectors. This large field-of-view makes ASKAP an unprecedented synoptic telescope poised to achieve substantial advances in SKA key science. The central core of ASKAP will be located at the Murchison Radio Observatory in inland Western Australia, one of the most radio-quiet locations on the Earth and one of the sites selected by the international community as a potential location for the SKA.

Following an introductory description of ASKAP, this document contains 7 chapters describing specific science programmes for ASKAP. In summary, the goals of these programmes are as follows:

- The detection of a million galaxies in atomic hydrogen emission across $75 \%$ of the sky out to a redshift of 0.2 to understand galaxy formation and gas evolution in the nearby Universe.

- The detection of synchrotron radiation from 60 million galaxies to determine the evolution, formation and population of galaxies across cosmic time and enabling key cosmological tests.

- The detection of polarized radiation from over 500,000 galaxies, allowing a grid of rotation measures at $10^{\prime}$ to explore the evolution of magnetic fields in galaxies over cosmic time.

- The understanding of the evolution of the interstellar medium of our own Galaxy and the processes that drive its chemical and physical evolution.

- The high-resolution imaging of intense, energetic phenomena by enlarging the Australian and global Very Long Baseline networks.

- The discovery and timing of a thousand new radio pulsars.

- The characterization of the radio transient sky through detection and monitoring of transient sources such as gamma ray bursts, radio supernovae and intra-day variables.

The combination of location, technological innovation and scientific program will ensure that ASKAP will be a world-leading radio astronomy facility, closely aligned with the scientific and technical direction of the SKA. A brief summary chapter emphasizes the point, and considers discovery space. 


\section{Introduction}

\section{Section authors: S. Johnston, R. Taylor}

The Australian SKA Pathfinder (ASKAP) is a next generation radio telescope on the strategic pathway towards the staged development of the Square Kilometre Array (SKA; see Schilizzi et al. 2007 for preliminary SKA specifications). The ASKAP project is international in scope and includes partners in Australia, Canada, the Netherlands and South Africa. This document, which concentrates on the science made possible with ASKAP was written as a joint collaboration between Australian and Canadian research scientists.

ASKAP has three main goals:

- to carry out world-class, ground breaking observations directly relevant to the SKA Key Science Projects,

- to demonstrate and prototype the technologies for the mid-frequency SKA, including field-of-view enhancement by focal-plane phased arrays on new-technology 12-metre class parabolic reflectors,

- to establish a site for radio astronomy in Western Australia where observations can be carried out free from the harmful effects of radio interference.

ASKAP is part of the Australian strategic pathway towards the SKA as outlined in the Australian SKA Consortium Committee's "SKA: A Road Map for Australia" document. ASKAP is seen as '...a significant scientific facility, maintaining Australia's leading role within the SKA partnership and addressing key outstanding computational/calibration risk areas ...'. SKA programs were given the highest priority for Australian radio astronomy in the 2006-2015 Decadal Plan for Astronomy.

In Canada the partnership in development and construction of ASKAP forms part of the Canadian SKA program, funded as part of one of the top priorities for future facilities in the Canadian Long Range Plan for Astronomy. In November 2006 the President of National Research Council of Canada and the Chief Executive Officer of the CSIRO signed an agreement declaring their intention to collaborate in the realization of ASKAP.

\subsection{ASKAP and SKA Science}

The SKA will impact a wide range of science from fundamental physics to cosmology and astrobiology. The SKA Science Case "Science with the Square Kilometre Array" (Carilli \& Rawlings 2004) identifies compelling questions that will be addressed as key science by the SKA:

- understanding the cradle of life by imaging the environments of the formation of earth-like planets, the precursors to biological molecules, and carrying out an ultrasensitive search for evidence of extra-terrestrial intelligence,

- carrying out fundamental tests of the theory of gravity by using radio waves to measure the strong space-time warp of pulsars and black holes and timing of arrays of pulsars over large areas of the sky to detect long-wavelength gravity waves propagating through the Galaxy, 
- tracing the origin and evolution of cosmic magnetism by measuring the properties of polarized radio waves from galaxies over cosmic history,

- charting the cosmic evolution of galaxies and large-scale structure, the cosmological properties of the Universe and Dark Energy; the imaging of atomic hydrogen emission from galaxies and the cosmic web from the present to time of the first galaxies, and

- probing the dark ages and the epoch of reionization of the Universe when the first compact sources of energy emerged.

The technological innovation of ASKAP and the unique radio-quiet location in Western Australia will enable a powerful synoptic survey instrument that will make substantial advance on three of the SKA key science projects: the origin and evolution of cosmic magnetism, the evolution of galaxies and large scale structure, and strong field tests of gravity. ASKAP will not only demonstrate a range of SKA technologies, it will also pathfind the scientific journey to SKA key science. The headline science goals for ASKAP are:

- The detection of a million galaxies in atomic hydrogen emission across $75 \%$ of the sky out to a redshift of 0.2 to understand galaxy formation and gas evolution in the nearby Universe.

- The detection of synchrotron radiation from 60 million galaxies to determine the evolution, formation and population of galaxies across cosmic time and enabling key cosmological tests.

- The detection of polarized radiation from over 500,000 galaxies, allowing a grid of rotation measures at $10^{\prime}$ to explore the evolution of magnetic fields in galaxies over cosmic time.

- The understanding of the evolution of the interstellar medium of our own Galaxy and the processes that drive its chemical and physical evolution.

- The high-resolution imaging of intense, energetic phenomena by enlarging the Australian and global Very Long Baseline networks.

- The discovery and timing of a thousand new radio pulsars.

- The characterization of the radio transient sky through detection and monitoring of transient sources such as gamma ray bursts, radio supernovae and intra-day variables.

\subsection{System Parameters}

Table 1 gives the ASKAP system parameters. The first column gives the parameter with the second column listing the symbol used in the equations in this section. The strawman (or base model) parameters for ASKAP are given in the third column of Table 1 and these strawman assumptions have been used throughout this paper. Likely upgrade or expansion paths include the addition of further dishes and/or the cooling of the focal plane array elements to provide a lower system temperature. Parameters for this expansion path are listed in the column 4 of Table 1.

ASKAP is designed to be a fast survey telescope. A key metric in this sense is the survey speed expressed in the number of square degrees per hour to a given sensitivity. Survey speeds and sensitivity for an interferometer like ASKAP have been derived elsewhere (e.g. Johnston \& Gray 2006) and the full derivation will not be shown here. 
Table 1 System parameters for ASKAP

\begin{tabular}{lccc}
\multicolumn{1}{c}{ Parameter } & Symbol & Strawman & Expansion \\
\hline \hline & $N$ & 30 & 45 \\
Number of Dishes & $N$ & 12 & 12 \\
Dish Diameter (m) & $A$ & 113 & 113 \\
Dish area $\left(\mathrm{m}^{2}\right)$ & & 3393 & 5089 \\
Total collecting area $\left(\mathrm{m}^{2}\right)$ & $\epsilon_{a}$ & 0.8 & 0.8 \\
Aperture Efficiency & $T$ & 50 & 35 \\
System Temperature (K) & & 30 & 30 \\
Number of beams & $F$ & 30 & 30 \\
Field-of-view (deg $\left.{ }^{2}\right)$ & & $700-1800$ & $700-1800$ \\
Frequency range (MHz) & $B$ & 300 & 300 \\
Instantaneous Bandwidth (MHz) & & 16000 & 16000 \\
Maximum number of channels & & 2000 & 400,8000 \\
Maximum baseline (m) & & & \\
\hline
\end{tabular}

To recap, the time $t$ required to reach a given sensitivity limit for point sources $\sigma_{s}$ is

$$
t=\left(\frac{2 k T}{A N \epsilon_{a} \epsilon_{c}}\right)^{2} \frac{1}{\sigma_{s}^{2} B n_{p}}
$$

where all parameters have their SI units. $B$ is the bandwidth, $n_{p}$ the number of polarizations, $A$ is the collecting area of a single element, $N$ is the number of elements and $\epsilon_{a}$ and $\epsilon_{c}$ represent dish and correlator efficiencies. The system temperature is $T$ with $k$ being the Boltzmann constant. The number of square degrees per second that can be surveyed to this sensitivity limit is

$$
S S_{s}=F B n_{p}\left(\frac{A N \epsilon_{a} \epsilon_{c} \sigma_{s}}{2 k T}\right)^{2}
$$

where $F$ is the field of view in square degrees. The surface brightness temperature survey speed is given by

$$
S S_{t}=F B n_{p}\left(\frac{\epsilon_{c} \sigma_{t}}{T}\right)^{2} f^{2} \epsilon_{s}^{-2}
$$

where now $\sigma_{t}$ denotes the sensitivity limit in $\mathrm{K}$, and $f$ relates to the filling factor of the array via

$$
f=\frac{A \epsilon_{a} N \Omega \epsilon_{s}}{\lambda^{2}}
$$

Here, $\epsilon_{s}$ is a 'synthesised aperture efficiency' which is related to the weighting of the visibilities and is always $\leq 1$.

There is interplay between these parameters when trying to maximise the survey speed for a given expenditure. For the majority of the science that will be considered here, the value of $S S_{s}$ and $S S_{t}$ are critical parameters, although the instantaneous sensitivity is also important. Although these equations are useful, they are not the entire story. For example, the effects of good uv coverage on the image quality do not appear in the equations. Furthermore, one should also not neglect the total bandwidth available for a spectral line survey. If the total bandwidth is insufficient to cover the required bandwidth of a given survey, the survey speed suffers as a result of having to repeat the same sky with a different frequency setting. 
The success of ASKAP is predicated on the focal plane array technology living up to expectations. In particular, technical challenges on achieving a low system temperate over a wide bandwidth, the ability to perform high dynamic range imaging, and polarization calibration of a wide field of view have yet to be met. It is not within the scope of this paper to investigate these issues in any detail. Rather, we note that much activity is taking place on all these challenges and the notable early success of the Westerbork APERTIF focal plane array (Verheijen et al. 2008) is encouraging.

In Table 2 we list values of the sensitivity and survey speed for different 'typical' surveys for both the strawman and the expansion parameters. The first line gives a continuum survey where the entire $300 \mathrm{MHz}$ of bandwidth is exploited and a desired sensitivity of $100 \mu \mathrm{Jy}$ is required. The second entry gives a spectral line survey, with the third line listing a surface brightness survey needing to reach $1 \mathrm{~K}$ rms over a $5 \mathrm{kHz}$ channel under the assumption of a $1^{\prime}$ resolution. The expansion option for ASKAP offers a factor of almost 5 improvement over the strawman design.

Table 2 Sensitivity and survey speeds for ASKAP

\begin{tabular}{lcc}
\hline \multicolumn{1}{c}{ Parameter } & Strawman & Expansion \\
\hline Continuum survey speed $(300 \mathrm{MHz}, 100 \mu \mathrm{Jy})$ & 250 & $1150 \mathrm{deg}^{2} / \mathrm{hr}$ \\
Line survey speed $(100 \mathrm{kHz}, 5 \mathrm{mJy})$ & 209 & $960 \mathrm{deg}^{2} / \mathrm{hr}$ \\
Surface brightness survey speed $\left(5 \mathrm{kHz}, 1 \mathrm{~K}, 1^{\prime}\right)$ & 18 & $83 \mathrm{deg}^{2} / \mathrm{hr}$ \\
Point source sensitivity $(1 \mathrm{MHz}, 1 \mathrm{mJy})$ & 1290 & $280 \mathrm{sec}$ \\
\hline
\end{tabular}

\subsection{Comparison with Other Instruments}

The large field-of-view makes ASKAP an unprecedented synoptic radio telescope, achieving survey speeds not available with any other telescope. ASKAP will routinely image very large areas of the sky to sensitivities currently only achievable with current instruments in very small areas.

The survey speed of ASKAP exceeds that of the Parkes 20-cm multibeam receiver, the Very Large Array (VLA) and the Giant Metre Wave Telescope (GMRT) by more than an order of magnitude for spectral line surveys in the $\mathrm{GHz}$ band. In continuum, ASKAP can survey the sky some 50 times faster than the NVSS survey carried out by the VLA over a decade ago.

As an interferometer ASKAP provides low-frequency imaging not otherwise available at the other major Southern Hemisphere interferometric array, the Australia Telescope Compact Array (ATCA). Even for single pointings, it exceeds the ATCA sensitivity at $1400 \mathrm{MHz}$ and with baselines extending beyond $6 \mathrm{~km}$ will have enhanced resolution. In terms of survey speed however, it gains by large factors for both line, continuum and surface brightness sensitivity and will be comparable to planned facilities in the Northern hemisphere such as the Allen Telescope Array (ATA) in the USA and APERTIF in the Netherlands.

The survey speeds of ASKAP (with the strawman parameters), the 350-dish ATA and APERTIF are almost identical although achieved in different ways. The ATA achieves its survey speed through a large number of small dishes each with a singlepixel feed. APERTIF will have a focal plane array system on larger dishes. The presence 
of telescopes with similar survey speed in the northern hemisphere makes for excellent complementarity to ASKAP.

\subsection{Configuration of ASKAP}

A number of science projects (pulsar surveys, Galactic H I, low surface brightness mapping) require a highly compact array configuration in order to increase the surface brightness survey speed (see equations 3 and 4). On the other hand science such as continuum and transients require long baselines both to overcome the effects of confusion and to obtain accurate positions for identifications at other wavelengths. In the middle is the extragalactic H I survey which needs moderate resolution to avoid over-resolving the sources. With a total of only 30 dishes it is difficult to achieve all these requirements simultaneously, although with 45 dishes a hybrid array becomes a possibility.

It is envisaged that three array configurations will be available. It is likely that the initial configuration will be the medium-compact configuration with maximum baseline length of $\sim 2 \mathrm{~km}$. Following a $\sim 2$ yr period, the configuration would then change to either an extended configuration (maximum baselines of $8 \mathrm{~km}$ ) followed by a highly compact configuration (maximum baseline of $400 \mathrm{~m}$ ) or vice-versa depending on scientific priorities. The configuration studies inckude consideration of simultaneous observations for different science, and the operational plan under development has this aspect in mind.

\subsection{Location of ASKAP}

The central core of ASKAP will be located at the Murchison Radio Observatory in inland Western Australia, one of the most radio-quiet locations on the Earth and one of the sites selected by the international community as a potential location for the SKA. The approximate geographical coordinates of the site are longitude $116.5^{\circ}$ and latitude $-26.7^{\circ}$. The southern latitude of ASKAP implies that the Galactic Centre will transit overhead and the Magellanic Clouds will be prominent objects of study. The choice of site ensures that ASKAP will be largely free of the harmful effects of radio interference currently plaguing the current generation of telescopes, especially at frequencies around $1 \mathrm{GHz}$ and below. Being able to obtain a high continuous bandwidth at low frequencies is critical to much of the science described in this document.

A significant component of ASKAP will be the construction of a multi-element outstation located in New South Wales at a distance of $\sim 3000 \mathrm{~km}$ from the core site. The exact location of the outstation remains to be decided at the time of writing of this document.

\subsection{Document Outline}

Following international science meetings held at the ATNF in April 2005 and March 2007, seven main science themes have been identified for ASKAP. These are extragalactic H I science, continuum science, polarization science, Galactic and Magellanic science, VLBI science, pulsar science and the radio transient sky. Each science theme has its 
own chapter in this document, with the final chapter as a brief summary. Lead authors from both Australian and Canadian institutions were allocated for each chapter; these lead authors had the opportunity to get input from other contributing authors on the various subsections of their chapter. The authorship of this document includes all lead and contributing scientists. 
2 Extragalactic $H_{\text {I }}$ Science

Lead authors: L. Staveley-Smith, U.-L. Pen

Contributing authors: C. Baugh, C. Blake, R. Braun, J. Darling, V. Kilborn, B. Koribalski, C. Power, E. Sadler

\subsection{Summary}

Understanding how galaxies form and evolve is one of the key astrophysical problems for the 21st century. Since neutral hydrogen ( $\mathrm{HI}$ ) is a fundamental component in the formation of galaxies, being able to observe and model this component is important in achieving a deeper understanding of galaxy formation. Widefield H I surveys using the next generation radio telescopes such as ASKAP and ultimately the SKA will allow unprecedented insights into the evolution of the abundance and distribution of neutral hydrogen with cosmic time, and its consequences for the cosmic star formation, the structure of galaxies and the Intergalactic Medium. ASKAP will provide powerful tests of theoretical galaxy formation models and improve our understanding of the physical processes that shaped the galaxy population over the last $\sim 7$ billion years.

\subsection{ASKAP H i Surveys}

ASKAP excels as a survey telescope as it will be able to spend long periods of time integrating on large areas of sky, resulting in the detection of large numbers of galaxies. Two compelling H I surveys are:

- A shallow hemispheric H I survey lasting a year. This would result in the detection of over 600,000 galaxies, or two orders of magnitude greater than HIPASS, the H I Parkes All-Sky Survey (Meyer et al. 2004). The typical survey depth would be $z \sim 0.05$ with massive galaxies detected out to $z \sim 0.15$ (see Figure 1 ).

- A deep 'pencil-beam' survey covering a single pointing, also lasting a year. Although this would only detect 100,000 galaxies in the example of a non-evolving H I mass function shown in Figure 2, the typical depth would be $z \sim 0.2$ with massive galaxies detected out to $z \sim 0.7$.

H I surveys are best carried out with compact configurations. However, the ability to resolve detections greatly increases understanding of the physical properties of galaxies and galaxy populations. A configuration which results in a resolution of around $30^{\prime \prime}$ at $z=0$ with a Gaussian natural beam appears a reasonable compromise (Staveley-Smith 2006). However, some science such as imaging the Cosmic Web requires greater column density sensitivity and short baselines, such as might be achieved using a reconfigurable array.

\subsection{Cosmic Neutral Gas Density}

Neutral atomic hydrogen is the most abundant element in the Universe and it plays a fundamental role in galaxy formation, principally as the raw material from which 


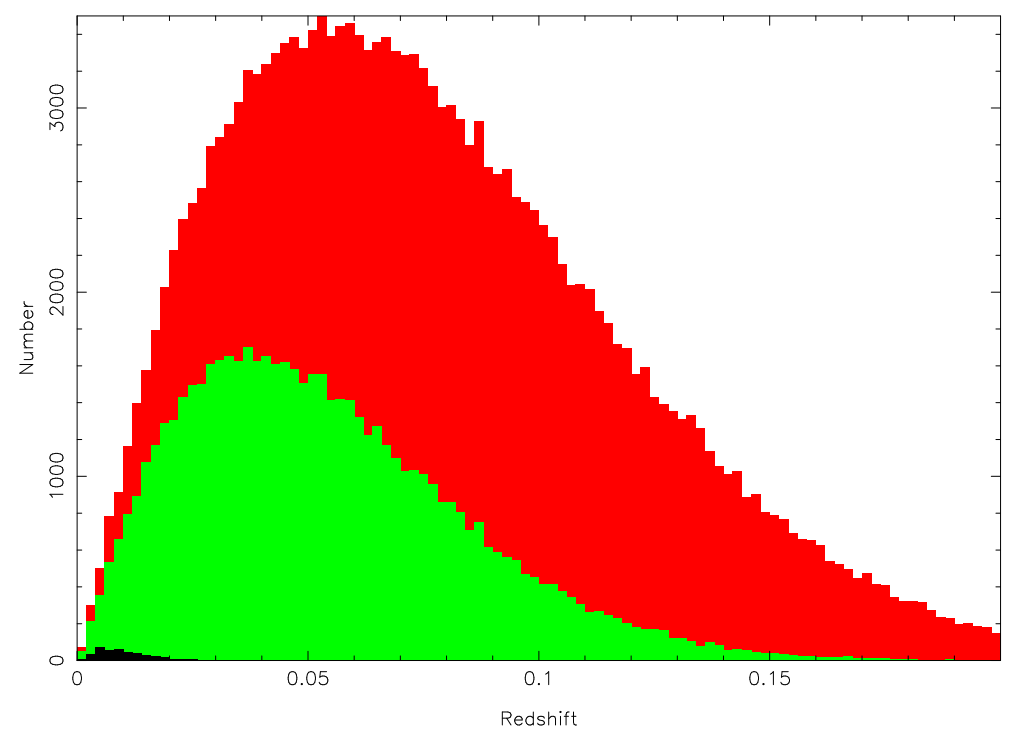

Fig. 1 Number of galaxies above $5 \sigma$ as a function of redshift bin for a shallow ASKAP $\mathrm{H}$ I survey lasting a year, covering the southern hemisphere, compared with HIPASS HICAT (Meyer et al. 2004). The simulation assumes the Zwaan et al. (2005) HIPASS mass function, and assumes a bivariate relation between $\mathrm{H}$ I mass and velocity width similar to that deduced from HIPASS. The number of predicted detections is over 1,800,000 for the expansion option (red histogram) and $\sim 600,000$ (green histogram) for the strawman option. The simulations plotted refer to a compact configuration - the number of detections sharply reduces for angular resolutions below $1^{\prime}$.

stars are made. At any given time we expect that the amount of neutral hydrogen in a galaxy will be determined by the competing rates at which $\mathrm{H} \mathrm{I}$ is depleted (by star formation and various feedback processes) and replenished (by accretion of cold gas from its surroundings and other feedback processes). Understanding how the abundance and distribution of $\mathrm{H} \mathrm{I}$ in the Universe evolves with redshift therefore provides us with important insights into the physical processes that drive the growth of galaxies and is a powerful test of theoretical galaxy formation models (e.g. Baugh et al. 2004).

The cosmic H I mass density $\Omega_{\mathrm{HI}}$ provides a convenient measure of the abundance of neutral hydrogen at a given epoch. Estimates of $\Omega_{\mathrm{HI}}$ at high redshifts $(z \gtrsim 1.5)$ can be deduced from QSO absorption-line systems in general and damped Lyman- $\alpha$ (DLA) systems in particular. DLA systems contain the bulk of $\mathrm{H}$ I at high redshifts and imply that $\Omega_{\mathrm{HI}} \simeq 10^{-3}$ (e.g. Peroux et al. 2003, Prochaska et al. 2005, Rao et al. 2006). At low-to-intermediate redshifts, however, the only known way to measure $\Omega_{\mathrm{HI}}$ accurately is by means of large-scale $\mathrm{H}$ i $21-\mathrm{cm}$ surveys.

This has been possible at $z \lesssim 0.04$ using the H I Parkes All-Sky Survey HIPASS (Meyer et al. 2004), which mapped the distribution of $\mathrm{H}$ in the nearby Universe that is observable from Parkes. HIPASS data have allowed accurate measurement of the local H I mass function (HIMF) and the H I density of galaxies, $\Omega_{\mathrm{HI}}$ (Zwaan et al. 2003, 2005). However, few measurements of the HIMF and $\Omega_{\mathrm{HI}}$ using H I $21-\mathrm{cm}$ emission have been possible over the redshift range $0.04 \lesssim z \lesssim 1$ because of insufficient sensitivity of current-generation radio telescopes (though see Lah et al. 2007), and so next generation telescopes such as ASKAP and ultimately the SKA will be required. 


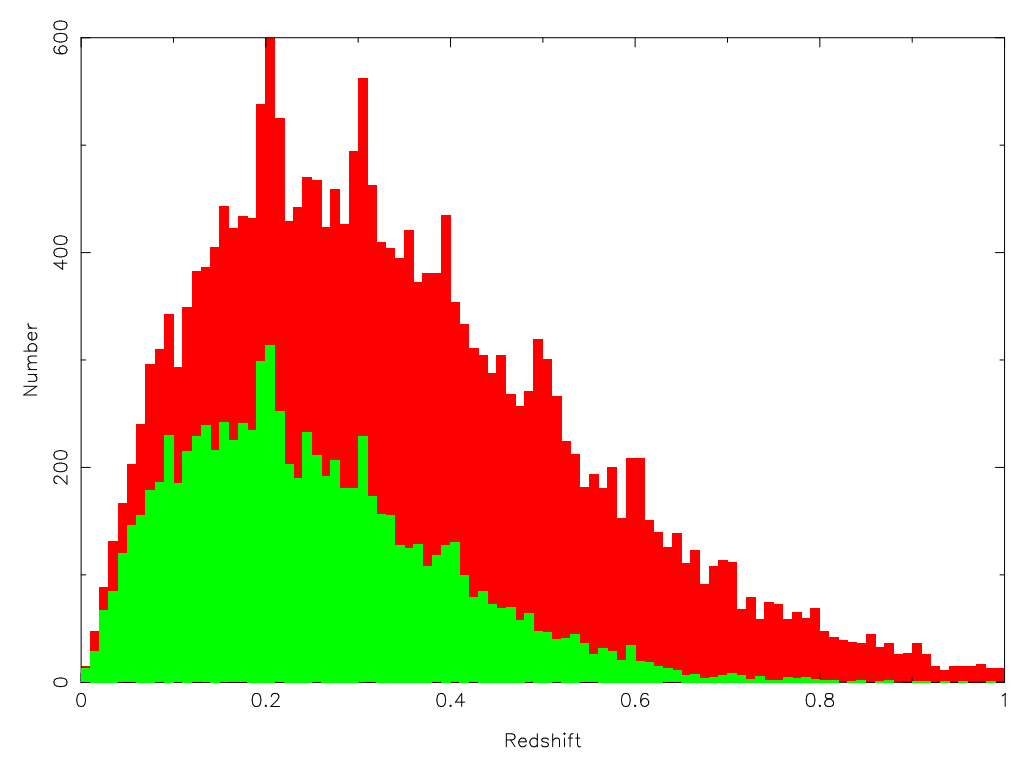

Fig. 2 Number of galaxies above $5 \sigma$ as a function of redshift bin for a deep ASKAP H I survey lasting a year, covering a single pointing. The simulation is similar to that for the allsky survey and assumes a non-evolving $\mathrm{H}$ I mass function. The number of predicted detections is $\sim 226,000$ for the expansion option (red histogram) and $\sim 99,000$ for the strawman option (green histogram). There is less dependence on angular resolution than for the shallow all-sky survey. Standard cosmology is assumed (flat Universe, $\mathrm{H}_{o}=75 \mathrm{~km} \mathrm{~s}^{-1} \mathrm{Mpc}^{-1}, \Omega_{m}=0.3$, $\left.\Omega_{\Lambda}=0.7\right)$.

\subsubsection{Semi-Analytic Galaxy Formation Models}

It is possible to use the semi-analytical galaxy formation code such as GALFORM (Cole et al. 2000) to investigate the predicted evolution with redshift of the HIMF and $\Omega_{\mathrm{HI}}$. Semi-analytical models provide a powerful theoretical framework within which we can explore the physical processes (e.g. star formation, the release of energy from $\mathrm{SNe}$ and AGN, referred to as "feedback") that drive the formation of galaxies and shape their observable properties (see Baugh 2006 for a comprehensive overview of semianalytical modelling). Numerical simulators call these processes "sub-grid physics". Due the current lack of a detailed understanding of the relevant physics, these phenomena are modelled with recipes or rules which contain parameters whose values can only be set so that appropriate subset of observational data are reproduced in local galaxy populations, e.g. the optical or infrared luminosity functions (see Cole et al. 2000).

For comparison, we consider two of the most successful models implemented in GALFORM: the one proposed by Baugh et al. (2005), which reproduces the abundances of Lyman-break and sub-millimetre galaxies at high redshifts; and the model of Bower et al. (2006), which recovers the observed break in the luminosity function at bright magnitudes particularly well and also matches the evolution of the stellar mass function. Both of these are regarded as viable models. They share many common ingredients but differ in some important aspects of the sub-grid physics relating to the formation of massive galaxies. In the Bower et al. model, AGN heating suppresses 
cooling flows in massive haloes. In the Baugh et al. model, the quenching of massive galaxy formation is achieved with a $\mathrm{SNe}$ driven wind which ejects cold gas from intermediate mass haloes, thereby removing this material from the low-redshift stages of the hierarchy. A further key difference between the models is that Baugh et al. assume a top-heavy IMF for bursts of star formation triggered by galaxy mergers.

\subsubsection{Evolution of $\Omega_{\mathrm{HI}}$ with Redshift}

In Figure 3, we compare the predictions of the Baugh et al. "top-heavy IMF" and the Bower et al. "AGN-heating" models with current observational estimates of the cosmic H I mass density at different redshifts. Here we express $\Omega_{\mathrm{HI}}$ as the mean $\mathrm{HI}$ mass density $\rho_{\mathrm{HI}}$ normalised by the critical density $\rho_{\mathrm{cr}}(0)$ at $z=0$.

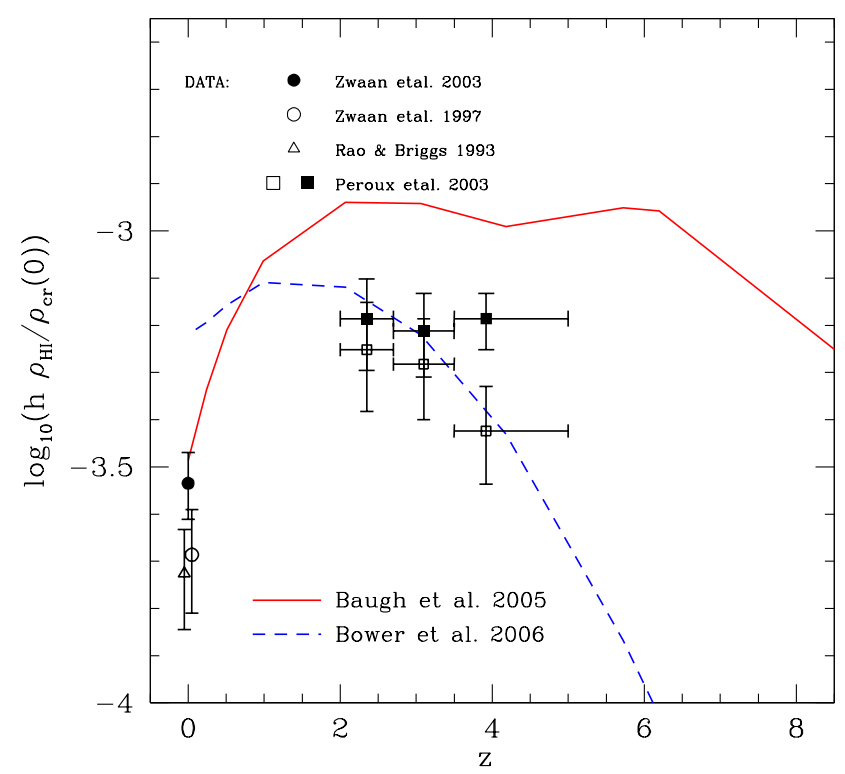

Fig. 3 Redshift evolution of the mass density of H I (expressed in units of the present day critical density). Predictions based on the Baugh et al. (2005) "top-heavy IMF" and Bower et al. (2006) "AGN-heating" models are shown by solid and dashed lines respectively. We show also recent observational estimates of the H I density at the present day (Rao \& Briggs 1993, Zwaan et al. 1997, 2003) and at higher redshifts (Peroux et al. 2003).

At $z=0$, we find that the Baugh et al. model predicts a cosmic H I mass density which is in good agreement with the HIPASS estimate obtained by Zwaan et al. (2003). In contrast, the Bower et al. model overpredicts the cosmic H I mass density by a factor of $\sim 2$. This difference arises because the Baugh et al. model was constrained to match both the $z=0$ B-band luminosity function and the observed gas fraction 
versus luminosity relation for late-type galaxies. Bower et al. attached less weight to reproducing the gas content of local spirals when setting the parameters of their model. The gas fractions in this model are somewhat higher than observed and so as a result, the Bower et al. model tends to overpredict local global density of cold gas.

At $z>0$, we find that the Baugh et al. model predicts a cosmic H I mass density that increases by a factor of $\sim 3$ between $0 \lesssim z \lesssim 2$ before rolling over and assuming a roughly constant value of $\sim 10^{-3}$ at $z \gtrsim 2$. The bump at $z \simeq 6$ reflects the treatment of the effect of photoionisation. At $z<6$, cooling is suppressed in haloes with virial circular velocities $V_{c}<60 \mathrm{kms}^{-1}$. When compared to the observational data of Peroux et al. (2003) (taken from their estimates of $\Omega_{\mathrm{DLA}}$ divided by the mean molecular mass $\mu$ ), we find that the Baugh et al. model predicts a cosmic H I mass density that is systematically higher by $\sim 60 \%$ than is inferred from observations. In contrast, the Bower et al. model predicts a cosmic H I mass density that is in reasonable agreement with the observational data; it is roughly constant between $0 \lesssim z \lesssim 2$ before declining rapidly ${ }^{1}$ at $z \gtrsim 2$.

The ability of ASKAP to accurately probe redshifts out to $\sim 0.8$ will fill a significant gap in our understanding of $\mathrm{H}$ I evolution. Re-expressed as a function of cosmic time, Figure 4 shows the significant gap in cosmic time (10 Gyr or so) between the $z=0$ observations and the higher redshift DLA observations, previously shown in Figure 3. ASKAP will be able to cover a significant fraction ( $70 \%$ or so) of this gap, will allow for much better overlap with low-redshift DLAs, presently selected from surveys of MgII absorbers, significantly improve on 'stacked' $21 \mathrm{~cm}$ observations of the type being pioneered by Lah et al. (2007), and will allow the development of more refined models of galaxy evolution.

\subsection{Environmental Influence on the H I Content of Galaxies}

Environment plays an important part in the evolution of galaxies. The observation of Butcher \& Oemler (1978) that high redshift clusters contain a much higher percentage of spiral galaxies than present day clusters indicates the evolution and transformation of spirals in the highest density regions of the Universe. Further evidence is seen in the star formation rates of galaxies in clusters which are much lower than those galaxies of similar morphology in the field (e.g. Lewis et al. 2002). One outstanding question is whether spiral galaxies are somehow transformed into the present-day lenticular, or S0, galaxies. The mechanism for such a transformation is still not clear: stripping, interactions, and the cessation of star formation leading to the fading of a spiral disk have all been proposed.

$\mathrm{H}$ I observations of galaxies in different environments provide an insight into their evolution. One of the main mechanisms affecting a galaxy's evolution is stripping of the cold H I gas, and thus removing the fuel for future star formation. The effects of gas stripping and tidal interactions are readily observed in the $\mathrm{H} \mathrm{I}$ content and distribution of galaxies. The H I distribution in late-type galaxies typically extends further than the detectable optical emission, and thus is subject to gravitational forces of any nearby

1 Note that the Bower et al. (2006) predictions are based on Millennium Simulation merger trees (Springel et al. 2005), and the mass resolution of the simulation is such that the cold gas mass fraction is underestimated at high redshifts. 


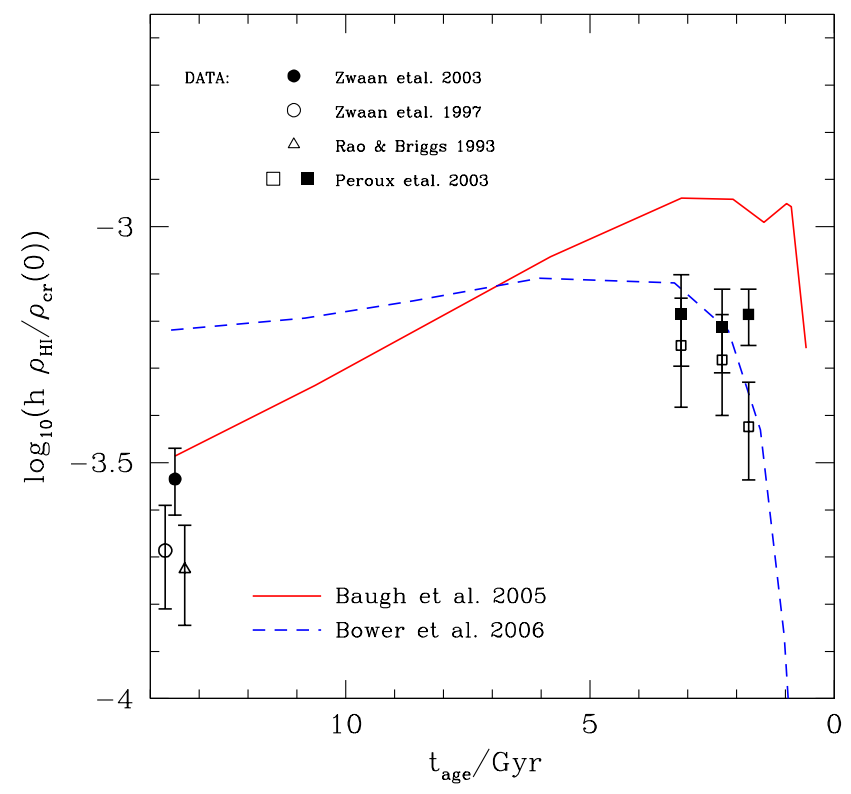

Fig. 4 Evolution of the mass density of $\mathrm{H}_{\mathrm{I}}$ (in units of the present day critical density) expressed as a function of cosmic time. Predictions based on the Baugh et al. (2005) "topheavy IMF" and Bower et al. (2006) "AGN-heating" models are shown by solid and dashed lines respectively. We show also recent observational estimates of the $\mathrm{H}$ I density at the present day (Rao \& Briggs 1993, Zwaan et al. 1997, 2003) and at higher redshifts (Peroux et al. 2003).

galaxies and group or cluster potentials, before we notice the effects in optical imaging. In addition, stripping processes that are prominent in massive clusters and groups with a hot intra-group medium, affect mainly the gas in galaxies.

Detailed observations of interacting galaxies can show large tidal tails, easily visible in the $\mathrm{HI}$ gas, sometimes before the optical observations show any sign of distortion (e.g. Yun et al. 1994). Galaxies in clusters typically contain less H I, or are 'H I deficient' when compared to optically similar galaxies in a low-density environment, suggesting that galaxies are somehow stripped of $\mathrm{HI}$ as they enter a dense environment (e.g. Solanes et al. 2001). Because of its wide field of view and ability to simultaneously image huge numbers of objects in a range of physical environments, ASKAP will be excellent for investigating the effect of environment on the evolution of galaxies.

There are a number of mechanisms that can strip H I gas from galaxies. In dense clusters where there is a hot intra-cluster medium and high velocity dispersions, galaxies can be stripped of their H I via ram pressure stripping (e.g. Gunn \& Gott 1972). Observational evidence of ram pressure stripping is seen in H I observations of spiral galaxies in the Virgo cluster (e.g. Kenney et al. 2004). 'Harassment' and 'starvation' are two other methods for galaxies to lose their gas. Harassment involves a high-speed encounter between two galaxies or a galaxy and the cluster potential, resulting in a loss of gas (Moore et al. 1996). Starvation involves the gas being slowly removed, perhaps 
due to a hot inter-cluster medium (ICM) - the effect is to stop star formation, as the supply of cold gas is thwarted (e.g. Treu et al. 2003). These three mechanisms are most effective in clusters, where there is a massive potential, and extended hot inter-galaxy gas. The other mechanism for galaxy transformation and gas removal is galaxy-galaxy interactions and mergers, a process which is most effective in the group environment.

The group environment is becoming increasingly recognised as one of the most important environments for processing, or changing the evolutionary path, of galaxies. For example, the star formation rate of galaxies begins to drop at the typical group densities (Gomez et al. 2003; Lewis et al. 2002). Over $70 \%$ of galaxies live in the group environment (Tully 1987), compared with less than $5 \%$ of galaxies that live in clusters (Dressler 1984). Groups have lower velocity dispersions than clusters, providing an ideal environment for interactions and mergers to take place (Mulchaey \& Zabludoff 1998). Preliminary results suggest that some galaxies that are members of loose groups may also be H I deficient (e.g. Kilborn et al. 2005; Omar \& Dwarakanath 2005; Chamaraux \& Masnou 2004); however the group environment is understudied compared to the cluster environment, so that the extent of this effect is yet unknown.

As the Hi content of galaxies changes with environment, so does the H I mass function. The Hi mass function is an essential ingredient in galaxy formation and evolution models, and determinations of the $\mathrm{H}$ I mass function provide a test for semianalytical models. The H I mass function of galaxies at low redshift is known to good precision, thanks to HIPASS (Zwaan et al. 2003). However, how the H I mass function changes with environment is less certain - Springob et al. (2005) found that the lowmass slope becomes shallower in denser environments, whereas Zwaan et al. (2005) found that it steepens in denser environments.

ASKAP will allow the first in-depth and homogeneous investigation of the $\mathrm{HI}$ content of galaxies with environment. While the cluster environment has been surveyed extensively in $\mathrm{H}$, the group environment, where most galaxies reside, remains largely unexplored. The sensitivity of previous H i surveys has been limited to Hi masses of about $10^{8}-10^{9} \mathrm{M}_{\odot}$, and for limited numbers of groups, so that effects such as ram pressure stripping in groups and $\mathrm{HI}_{\mathrm{I}}$ deficiency have not been quantified. The increased sensitivity of ASKAP over previous studies will allow the determination of the H I mass function down to much lower masses, and for greater number statistics when investigating the change in the $\mathrm{H}$ I mass function with environment. With a spatial resolution of $30^{\prime \prime}$, ASKAP will enable the closest galaxies to be mapped without recourse to follow-up observations - thus we will determine the H I morphology, and thus how the environment is affecting the galaxy, for a large, homogeneous number of nearby galaxies. Combining these data with optical, IR and continuum observations will for the first time allow a full picture of galaxy evolution in the local Universe.

\subsection{Imaging the Low Redshift Cosmic Web}

Extragalactic astronomy has traditionally focussed on the regions of extreme cosmic overdensity that we know as galaxies. Only in recent years has the realization emerged that galaxies do not dominate the universal baryon budget but are merely the brightest pearls of an underlying Cosmic Web. Filamentary components extending between the massive galaxies are a conspicuous prediction of high resolution numerical models of structure formation (e.g. Davé et al. 1999, 2001). Such calculations suggest that in the current epoch, cosmic baryons are almost equally distributed by mass amongst three 
components: (1) galactic concentrations, (2) a warm-hot intergalactic medium (WHIM) and (3) a diffuse intergalactic medium. These three components are each coupled to a decreasing range of baryonic density, namely $\rho_{H} / \overline{\rho_{H}}>10^{3.5}, 10-10^{3.5}$, and $<10$, and are correspondingly probed by QSO absorption lines with specific ranges of neutral column density: $N_{H I}>10^{18}, 10^{14}-10^{18}$, and $<10^{14} \mathrm{~cm}^{-2}$, as shown in Figure 5 . The neutral fraction is thought to decrease with decreasing column density from about $1 \%$ at $N_{H I} \sim 10^{17} \mathrm{~cm}^{-2}$, to less than $0.1 \%$ at $N_{H I} \sim 10^{13} \mathrm{~cm}^{-2}$. Although a very wide range of physical conditions can be found within galaxies, the WHIM is thought to be a condensed shock-heated phase of galaxy interaction debris with temperature in the range $10^{5}-10^{7} \mathrm{~K}$, while the diffuse IGM is predominantly photo-ionized with temperature near $10^{4} \mathrm{~K}$. A complicating factor to this simple picture is the growing suspicion that the gas accretion process, traced by the $\log \left(\mathrm{N}_{H I}\right)=14-18$ systems, may well occur in two rather different regimes (e.g. Binney 2004, Keres et al. 2005). Low to moderate mass galaxies $\left(\mathrm{M}_{V i r}<10^{12} \mathrm{M}_{\odot}\right)$ may experience primarily "coldmode" accretion $\left(\mathrm{T} \sim 10^{4.5} \mathrm{~K}\right.$ ) along filaments, while only more massive systems may be dominated by the more isotropic "hot-mode" accretion $\left(\mathrm{T} \sim 10^{5.5} \mathrm{~K}\right)$, which until recently was thought to be universal (e.g. Rees \& Ostriker 1977, Davé et al. 2001).
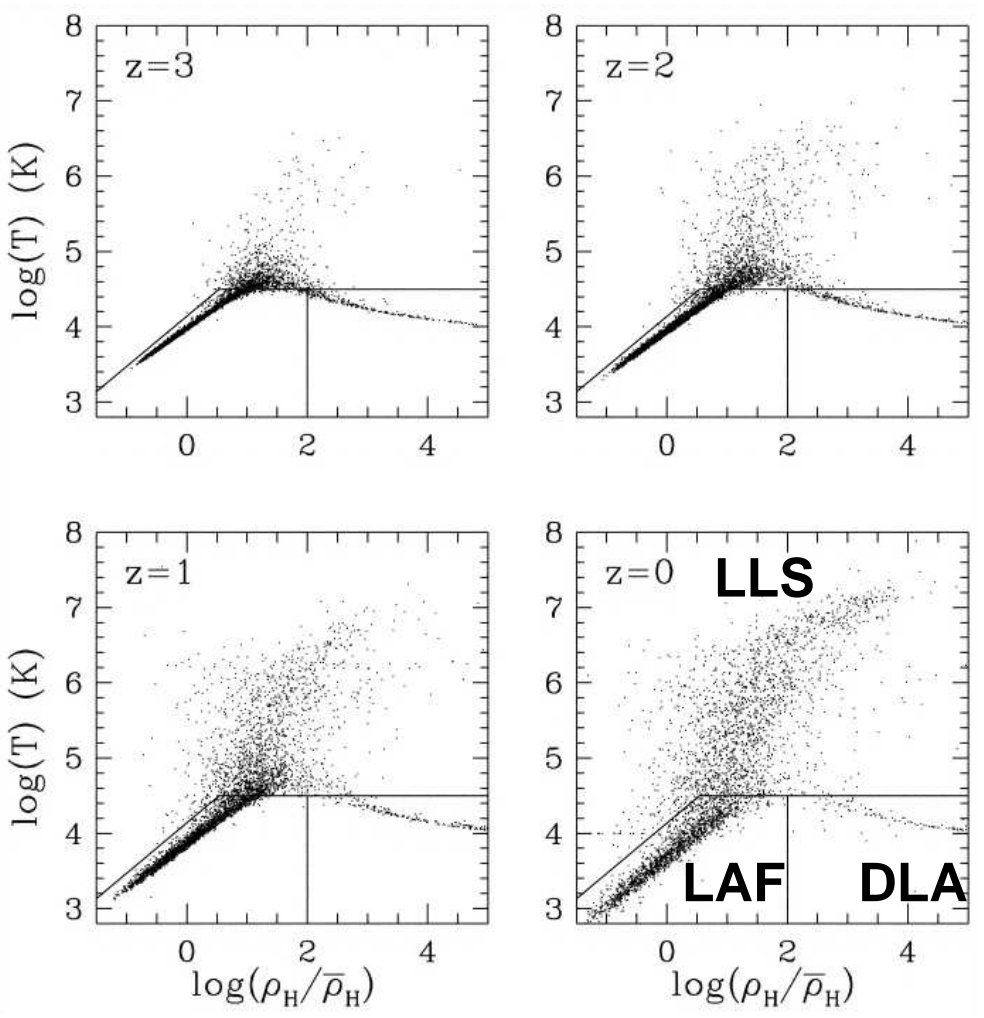

Fig. 5 The redshift evolution of overdense regions in a numerical simulation showing the increasing mass fraction with time of gas in the range $\mathrm{T}=10^{4.5}-10^{7} \mathrm{~K}$, the so-called WHIM (from Davé et al. 1999; reproduced with the kind permission of the authors). Also shown are the regimes of Damped Ly $\alpha$, Lyman Limit system and Ly $\alpha$ forest absorber systems. 
The strongest observational constraints on the Cosmic Web come from the statistics of the QSO absorption lines. Enough such QSO spectra have been obtained to allow good statistical determinations to be made of the rate of occurrence of intervening absorbers as function of their column density. By binning such data in redshift intervals, it has even been possible to gauge the cosmic evolution of intervening absorbers (e.g. Storrie-Lombardi \& Wolf 2000). Inter-galactic space has apparently become continuously tidier by about an order of magnitude from redshifts of several down to zero, with a decreasing cross-section of high column absorbers. At the current epoch we can now confidently predict that in going down from $\mathrm{H}$ I column densities of $10^{19} \mathrm{~cm}^{-2}$ (which define the current "edges" of well-studied nearby galaxies in H I emission) to $10^{17} \mathrm{~cm}^{-2}$, the surface area will increase by a factor of 30 . The critical observational challenge is crossing the "H I desert", the range of $N_{H I}$ from about $10^{19.5} \mathrm{~cm}^{-2}$ down to $10^{18} \mathrm{~cm}^{-2}$, over which photo-ionization by the intergalactic radiation field produces an exponential decline in the neutral fraction from essentially unity down to a few percent (e.g. Dove \& Shull 1994). Nature is kinder again to the H I observer below $\log \left(\mathrm{N}_{H I}\right)=18$, where the neutral fraction decreases only very slowly with $\log \left(\mathrm{N}_{H I}\right)$. The baryonic mass traced by this gas (with a $1 \%$ or less neutral fraction) is expected to be comparable to that within the galaxies, as noted above.

How are these diffuse systems distributed, what are their kinematics, and under what conditions does "cold-mode" or "hot-mode" accretion dominate? These are questions which can not be addressed with the QSO absorption line data. The areal density of suitable background sources is far too low to allow "imaging" of the intervening low column density systems in absorption. Direct detection of the free-free continuum or recombination line emission from the ionized gas has also proven well beyond the capabilities of current X-ray and optical instrumentation. For example the expected $\mathrm{H} \alpha$ emission measure is only $\sim 5 \times 10^{-4} \mathrm{~cm}^{-6} \mathrm{pc}$. The very best current $\mathrm{H} \alpha$ imaging results reach down to emission measures of $\sim 0.1 \mathrm{~cm}^{-6} \mathrm{pc}$, which is still orders of magnitude removed from what would be needed.

Although conventional imaging in $\mathrm{H}$ i has not typically reached column densities below about $10^{19} \mathrm{~cm}^{-2}$, this is not a fundamental limitation. Long integrations with an (almost-)filled aperture can achieve the required brightness sensitivity to permit direct imaging of the small neutral fraction within the Cosmic Web filaments between galaxies. The first detection of such diffuse filaments in the extended environment of M31 was made by Braun \& Thilker (2004). This was accomplished by utilizing total power measurements made with the fourteen $25 \mathrm{~m}$ dishes of the WSRT. Although the angular resolution is low ( $49^{\prime}$ corresponding to $11 \mathrm{kpc}$ at the M31 distance) the column density sensitivity is very high ( $\mathrm{rms}$ of $4 \times 10^{16} \mathrm{~cm}^{-2}$ over $17 \mathrm{~km} \mathrm{~s}^{-1}$ ). A diffuse filament is detected connecting the systemic velocities of M31 to M33 (at a projected separation of $200 \mathrm{kpc}$ ) and also extending away from M31 in the anti-M33 direction as shown in Figure 6. This diffuse filament appears to be fueling denser gaseous streams and filaments in the outskirts of both galaxies. Peak neutral column densities within the filament only amount to some $3 \times 10^{17} \mathrm{~cm}^{-2}$.

The interaction zone of the diffuse filament with M31 has been studied in complementary surveys that permit calculation of the $\mathrm{H}$ I distribution function from $\mathrm{HI}$ emission measurements (rather than QSO absorption measurements) over an unprecedented range in $\log \left(\mathrm{N}_{H I}\right)=17.2$ to $\log \left(\mathrm{N}_{H I}\right)=21.9$ as shown in Figure 6. The H I distribution function of these structures agrees very well with that of the low redshift QSO absorption lines which are also plotted in the figure as filled circles with error bars. The predicted factor of 30 increase in surface covering factor for low $\mathrm{N}_{H I}$ emission 

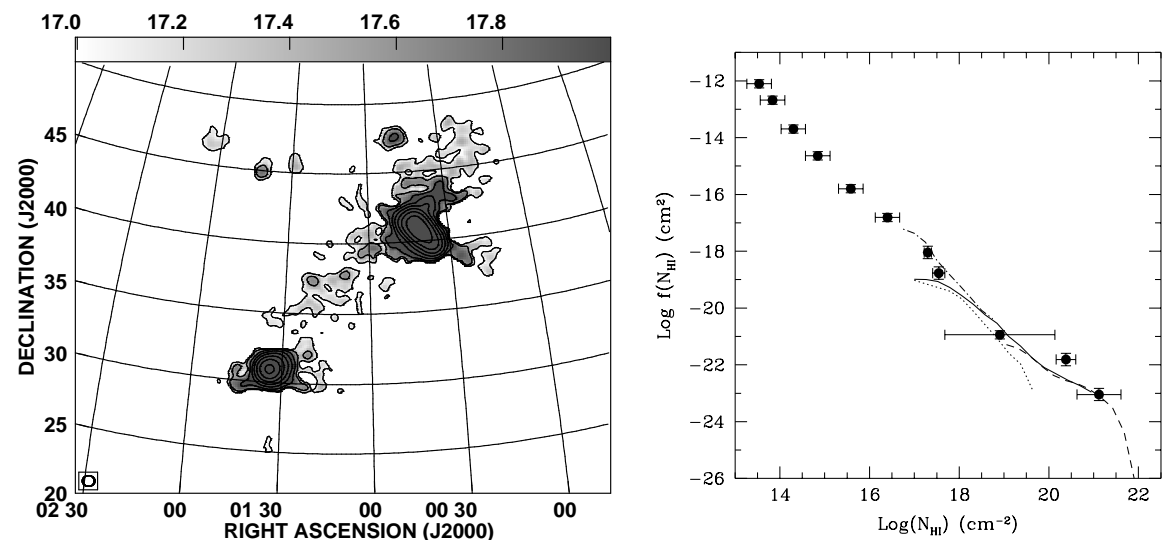

Fig. 6 A Cosmic Web filament (left) of H I gas connecting the nearby galaxies M31 and M33 together with the corresponding H I distribution function (right) from Braun \& Thilker (2004). The curves are the $\mathrm{H}$ I data, while the filled points represent QSO optical absorption line data. With sensitive $\mathrm{H}$ I observations the Lyman Limit absorption systems can be directly imaged in emission, despite their small neutral fraction, permitting direct study of the WHIM and its large reservoir of baryons.

has been observationally verified. In so doing, it has been possible to provide the first image of a Lyman Limit absorption system. The morphology and kinematics are fully in keeping with the Cosmic Web hypothesis outlined above. We are now in a position to witness the continuing gaseous fuelling of normal galaxies with direct imaging. This gas represents the most effective tracer of the last 5 Gyr or so of galaxy interaction history. In combination with optical/UV absorption line observations of the ionization state and metallicity it then becomes possible to quantify the mass budget and enrichment history of this major baryon reservoir.

Efforts are now underway to extend the study of Lyman Limit Systems in H I emission beyond the Local Group. The deepest practical survey at this time (involving more than 1000 hours of observing time with the WSRT) will reach an rms of $2 \times 10^{17} \mathrm{~cm}^{-2}$ over $20 \mathrm{~km} \mathrm{~s}^{-1}$ within a $35^{\prime} \times 3^{\prime}$ beam. This should permit the environments of several hundred galaxies to be probed to these depths for the first time. Some of the brightest Cosmic Web filaments can then be studied, when they fortuitously align with the elongated beam.

Similar surveys with an ultracompact ASKAP configuration will be truly groundbreaking. For example, squeezing the nominal performance 30 antennas into a $200 \mathrm{~m}$ configuration provides a circular $5^{\prime}$ beam, corresponding to better than $20 \mathrm{kpc}$ physical resolution out to distances of $14 \mathrm{Mpc}$, with an rms sensitivity of $2 \times 10^{17} \mathrm{~cm}^{-2}$ over $20 \mathrm{kms}^{-1}$ in a 12 hour integration over the entire $30 \mathrm{deg}^{2}$ FoV. A single 12 hour integration will thus be sufficient to detect the brightest knots within Cosmic Web filaments between major galaxies. Longer integrations, of 144 hours, will reach $5 \times 10^{16}$ $\mathrm{cm}^{-2}$ at this resolution, permitting routine detection of Lyman Limit System filaments throughout the Local Volume.

With the higher sensitivity expansion option, 45 antennas with 1.4 times better noise performance, it would then be possible to push Cosmic Web detection out to substantially larger distances. It would still be necessary to utilize an ultra-compact 
ASKAP Simulated HIMF Recovery

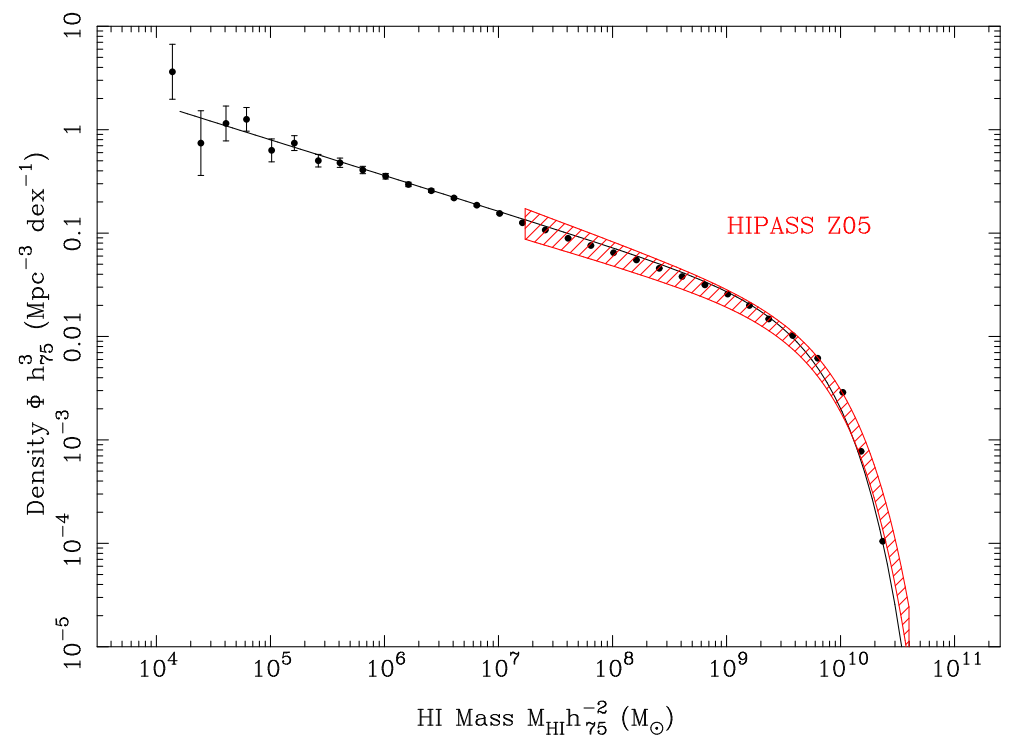

Fig. 7 An Hi mass function calculated from the simulation of an ASKAP all-sky shallow H I survey with the strawman configuration. The recovered mass function probes H I masses two orders of magnitude below those probed by HIPASS (Zwaan et al. 2005). The simulation considers only galaxies outside the Local Group.

configuration, of say $300 \mathrm{~m}$ diameter, yielding a $3.5^{\prime}$ beam, but this would probe the same low column densities noted above out to an effective distance of at least $20 \mathrm{Mpc}$, corresponding to a three times larger volume than accessible with the nominal ASKAP specifications.

\subsection{The Local Volume}

The 'Local Volume' (the sphere of radius $\sim 10$ Mpc centered on the Local Group) includes at least 500 known galaxies, many of which congregate in well-known groups like the Local Group, the relatively loose Sculptor Group and the more compact Centaurus A group. The fact that accurate distances (e.g. from TRGB (tip of the red giant branch) measurements obtained with the HST) can now be obtained quite easily for the majority of these galaxies makes this volume and its research so special and timely. The combination of accurate galaxy positions, radial velocities and independent distances allows us to create a true 3D view of the Local Universe with $1 \mathrm{D}$ velocities. This in turn leads to a detailed understanding of the local flow field, i.e. the Hubble flow and its dispersion. It also leads to a better understanding of the overall matter (dark and visible) distribution in and between galaxies as well as an accurate H I mass function at the low-mass end.

ASKAP will enormously contribute to our understanding of the Local Volume by revealing all gas-rich galaxies in the surveyed sky down to an H I mass of a few times $10^{5} \mathrm{M}_{\odot}$, vastly surpassing HIPASS in sensitivity as well as both angular and velocity resolution. At the fringes of the Local Group, the sensitivity limit will be such that 
significant numbers of low-mass galaxies such as the recently discovered transition dwarf, Leo T (Irwin et al. 2007) are likely be found by ASKAP.

\subsection{H i Absorption-Line Surveys}

The $21 \mathrm{~cm}$ emission line of $\mathrm{HI}$ is extremely weak, and its detection at redshifts above $z \sim 0.1$ stretches even the largest existing radio telescopes. As a result, there have so far been only a few detections of H I emission from individual galaxies above $z \sim 0.1$, even in targeted searches with integration times of days to weeks.

The vast collecting area of the Square Kilometre Array (SKA) will allow it to detect $\mathrm{H}$ I in emission out to redshift $z \sim 3$ and, as noted earlier, integration times of up to a year will allow ASKAP to detect some galaxies out to redshifts exceeding $z \sim 0.5$. Until the advent of SKA, however, H I absorption-line spectroscopy offers a powerful alternative tool for investigating the neutral hydrogen content of normal galaxies over the full redshift range covered by ASKAP.

Although such investigations only probe galaxies which happen to lie along the lineof-sight to strong background radio sources, they can provide detailed information on the kinematics and mass of neutral gas in these galaxies (e.g. Kanekar \& Briggs 2004). As can be seen from Figure 3, most of our knowledge about the H I mass density in the high-redshift universe comes from optical absorption-line studies of QSOs, but such studies become extremely difficult below $z \sim 1.7$ because the Lyman $\alpha$ line can no longer be observed with ground-based optical telescopes. Blind H I absorption-line surveys at radio wavelengths can potentially bridge much of the gap in Figure 3. They have the additional advantage that the background radio source is likely to be an (optically) faint galaxy rather than a bright QSO, so follow-up optical studies of the $\mathrm{H}$ I absorbers will be relatively straightforward.

The large field of view and wide bandwidth of ASKAP will make it an excellent instrument for carrying out blind $\mathrm{H}$ I absorption-line surveys at $0<z<0$.7. Figure 8 shows an example of an $\mathrm{H}$ I absorption-line system at $z=0.4$ which would be easily detectable with ASKAP. Only a dozen or so H I absorption-line systems in the redshift range $0.1<z<1$ have so far been detected by radio telescopes (see Figure 9), because the small bandwidth of most current radio spectrometers makes blind-search techniques too time-consuming. Most previous studies have therefore targeted the small number of radio-loud QSOs already known to have optical absorption lines at a particular redshift .

A 12-hr integration with the Expanded ASKAP array should be able to make reliable detections of $\mathrm{H}$ I absorption lines with optical depth $\tau>0.1$ against background sources with continuum flux densities $\geq 50 \mathrm{mJy}$ (100 mJy for the strawman array). In contrast to $\mathrm{H}$ I emission-line surveys, the sensitivity of an $\mathrm{H}$ I absorption-line survey is roughly constant with redshift over the ASKAP frequency range. (Around $80 \%$ of continuum sources above 50 mJy lie at $z>0.8$, so can be considered as background objects.)

For the ASKAP redshift range $0<z<0.7$, we therefore estimate a detection rate of $2-4 \mathrm{HI}$ absorbers per $30 \mathrm{deg}^{2}$ field for a $12 \mathrm{hr}$ integration with a $300 \mathrm{MHz}$ spectral bandpass and $4-5 \mathrm{~km} \mathrm{~s}^{-1}$ channels. This estimate is somewhat uncertain at $z>0.1$ since it relies on an interpolation between the measured number densities of damped Lyman $\alpha$ (DLA) absorbers at $z \sim 0$ (Ryan-Weber et al. 2003) and $z>2$ (StorrieLombardi \& Wolfe 2000; Prochaska \& Herbert-Fort 2004). A blind H I absorption-line 


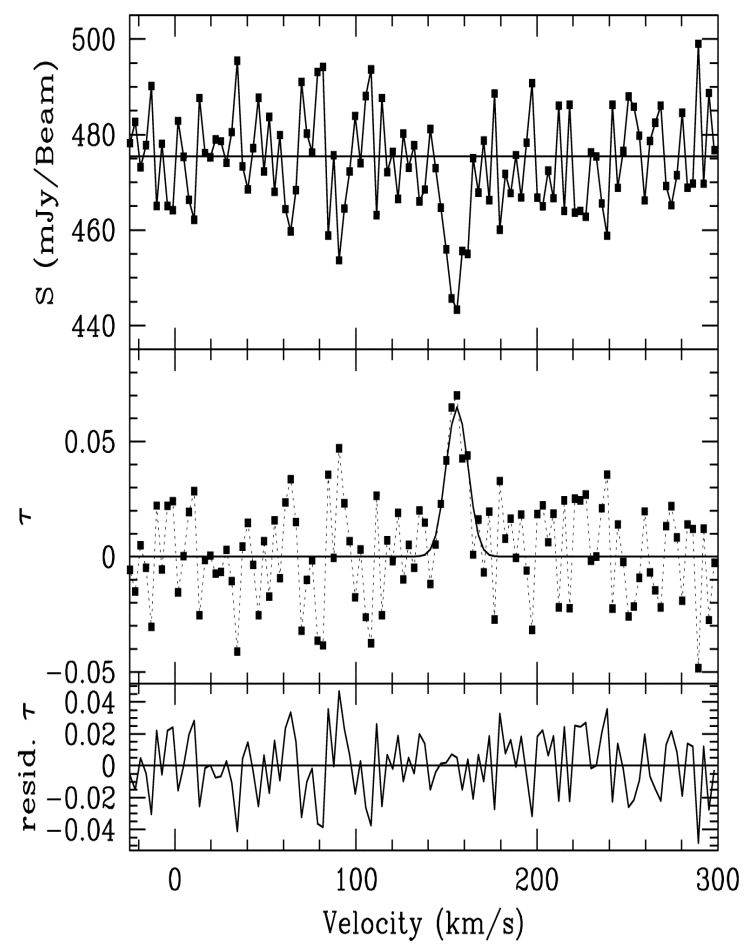

Fig. 8 Radio spectrum of the QSO B 1243-072 (Lane \& Briggs 2001; reproduced with the kind permission of the authors), showing an $\mathrm{H}$ I absorption line at $z=0.436$. The middle panel shows a Gaussian fit to the optical depth in the line profile $(\tau \sim 0.08)$, and the bottom panel shows the residuals in $\tau$ after subtracting the fitted profile.

survey of about $2000 \mathrm{deg}^{2}$ of sky at $z \sim 0.65-0.71$ will be carried out with the Molonglo radio telescope and SKAMP-II correlator in 2007-8, and should give a much better indication of the actual number and distribution of $\mathrm{H}$ I absorbers which will be observable by ASKAP.

For the redshift range $0<z<0.2$, a blind $\mathrm{H}$ I absorption-line survey can be done in parallel with the shallow all-sky $\mathrm{H}$ I emission-line survey and should yield at least a thousand or so absorption-line detections over the whole sky. At $z>0.3$, a separate absorption-line survey would be a valuable complement to the planned deep H I emission survey.

\subsection{Cosmology with H i Galaxies}

\subsubsection{Galaxy Clustering}

The clustering of galaxies encodes a wealth of cosmological and astrophysical information. In particular, the patterns of clustering reflect the manner in which a particular class of galaxies populates the underlying network of dark matter haloes, hence tracing its formation and evolution history. For example, it is well known that optically-selected red galaxies possess a higher clustering amplitude or "bias" than blue galaxies. This 


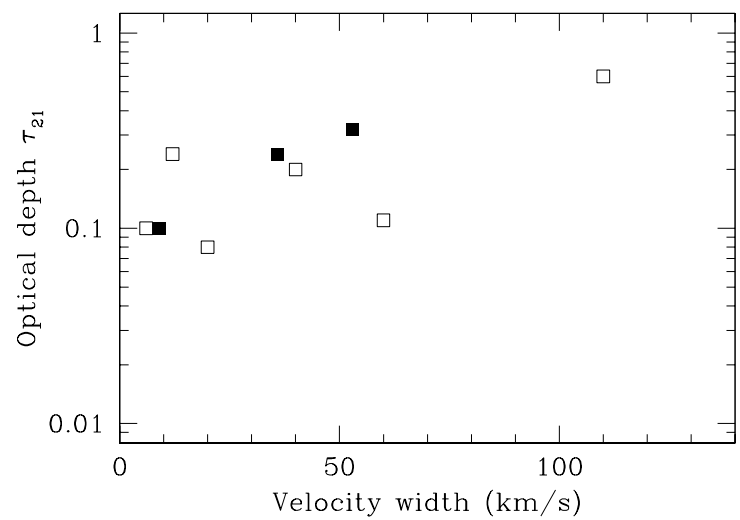

Fig. 9 Observed $\mathrm{H}$ i optical depth $\tau$ and velocity width of currently-known $\mathrm{H}$ i absorptionline systems in the redshift range $0.3<z<0.8$. Filled squares show the three detections from 'blind' surveys of strong continuum sources with single-dish telescopes (Brown \& Roberts 1973; Brown \& Mitchell et al. 1983; Darling et al. 2004); open squares are from targeted radio observations of QSOs at the redshifts of known optical absorption-line systems (Chengalur \& Kanekar 2000; Lane \& Briggs 2001; Kanekar et al. 2002).

reflects the tendency of red galaxies to reside in denser environments, or more massive dark matter haloes, which in turn reflects upon their formation mechanism. Furthermore, clustering strength also increases with optical luminosity.

These arguments have recently been quantified in the halo model for galaxy clustering. This model assumes that the mass function, clustering and radial profiles of the underlying dark matter haloes can be established accurately from N-body simulations. The clustering of the galaxies on top of these haloes is then described by the halo occupation distribution, $N(M)$, which specifies the average number $N$ of galaxies residing in a halo of mass $M$. This function is parameterized in a simple way, and fit to the observations. The successful performance of this analysis depends on detecting deviations from a power-law in the clustering correlation function, which is only possible for large galaxy surveys such as the 2-degree Field Galaxy Redshift Survey (2dFGRS, Colless et al. 2001) and the Sloan Digital Sky Survey (SDSS, York et al. 2000).

First measurements of the clustering of $\mathrm{H}$ I-emission galaxies have been performed using the HIPASS survey (Ryan-Weber 2006, Meyer et al. 2007, Basilakos et al. 2007). The result (from a simple power-law fit) is that $\mathrm{H}$ I-selected galaxies possess a very low clustering bias. This is expected because $\mathrm{H}$ I galaxies appear to live in under-dense environments, given that as these galaxies enter the denser cluster environment, the gas is stripped or exhausted via star formation.

As shown in Section 2.2, ASKAP can perform an H I galaxy redshift survey on the scale of the 2dFGRS. This will yield extremely accurate measurements of the clustering pattern as a function of galaxy mass and environment, which will form a key part of ASKAP's central portfolio of observations. The characteristic "halo-model" deviation from a power-law clustering correlation function will be detected, enabling the dark matter halo occupation distribution to be inferred. These clustering measurements will reflect on where $\mathrm{H}$ I galaxies form, and how the gas is stripped or exhausted in the vicinity of dense environments. As in Section 2.4, various processes can remove $\mathrm{H}$ I from galaxies: gravitational tidal effects, ram pressure stripping, or strangulations. The crosscorrelation of the $\mathrm{H}$ I galaxies with the distributions of clusters, groups and filaments 
will discriminate between these processes via the scales and densities at which they operate. The imprint of the redshift-space distortions will also be a key measurement, indicating how $\mathrm{H}$ I galaxies coherently flow into overdense regions. The work can be extended to higher redshifts $(z \approx 0.6)$ through deeper ASKAP pointings.

\subsubsection{ASKAP as an SKA Cosmology Demonstrator}

Future radio telescopes, culminating in the SKA, can potentially become the world's leading facilities for performing cosmological surveys. These advances are based on simple considerations of survey speed, which scales as $A^{2} \Omega$ for radio telescopes, where $A$ is collecting area and $\Omega$ is field-of-view, as opposed to $A \Omega$ for optical telescopes. The scaling with $A^{2}$ ensures that the SKA (which provides a 2 order of magnitude increase in collecting area compared to existing radio telescopes) can detect $\mathrm{H}$ I emission-line galaxies to redshift $z \sim 3$ in just a few hours integration. Such a redshift survey can be performed simultaneously with a deep continuum imaging survey, owing to the large telescope bandwidth. Furthermore, the goal for the SKA is a field-of-view $\Omega=200$ $\operatorname{deg}^{2}$ at $0.7 \mathrm{GHz}$, exceeding what is possible for an optical telescope by at least an order-of-magnitude.

Simple telescope and Hi mass function models demonstrate that the SKA can achieve an all-sky $\mathrm{H}$ I emission-line galaxy redshift survey containing $\sim 10^{9}$ galaxies to a $5 \sigma$ H I mass limit $\sim 5 \times 10^{9} M_{\odot}$ out to redshift $z \sim 1.5$. Such a survey would map a cosmic volume exceeding what is possible today by a factor of 100 . The dataset will allow transformational cosmological measurements, which form one of the Key Science Goals for the SKA. The patterns of galaxy clustering through this volume encode the properties of dark energy and dark matter haloes, the masses of neutrino species, and the physics of inflation. Baryon oscillations in the galaxy distribution can be used as a standard ruler to map out the cosmic expansion history. The ASKAP strawman option will provide useful cosmological measurements, However, as Figure 10 shows, the detection of the so-called 'baryonic acoustic oscillations' will probably only be possible with the expanded option and only on small spatial scales with the all-sky survey. This will preclude the investigation of 'dark energy' studies at higher redshift in a manner competitive with future and ongoing optical surveys such as 'WiggleZ' (Glazebrook et al. 2007). Nevertheless, ASKAP can pave the way for these future SKA surveys by resolving key issues of survey design and analysis:

- ASKAP can precisely measure the clustering properties of $\mathrm{H}$ I galaxies over a range of redshifts. This not only contains information about the manner in which gas in galaxies is assembled, but is also a major unknown for the calculation of systematic errors in future SKA baryon oscillation surveys. Future cosmological measurements will be limited by systematic errors rather than statistical errors - quantifying and controlling these systematic errors is crucial for extracting robust cosmological conclusions. The leading sources of systematic error for baryon oscillation surveys are the poorly-modelled effects of redshift-space distortions, halo bias and the nonlinear growth of structure. These processes all modify the underlying linear power spectrum which encodes the baryon oscillation signature, hampering our cosmological measurements. They can generically be described as "scale-dependent galaxy bias". ASKAP can measure this function, including the "halo occupation distribution" of these galaxies with respect to the underlying dark matter fluctuations.

- ASKAP can measure the H I mass function with unprecedented accuracy, including how this function varies with redshift (to $z \approx 0.8$ ) and environment. The SKA 
Power spectrum accuracy of $\mathrm{HI}$ redshift survey in 1 year

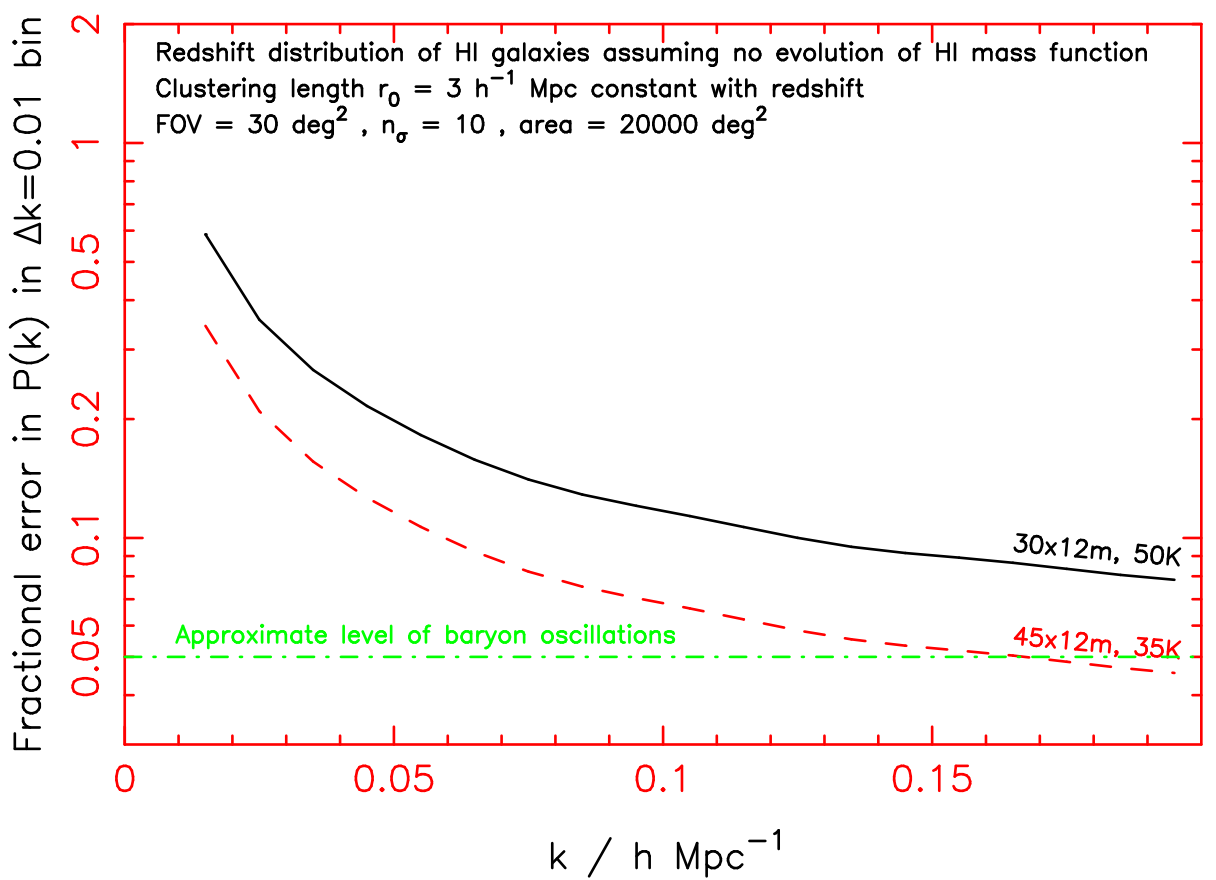

Fig. 10 The fractional error in the H I power spectrum $P(k)$ for a 1-yr hemispheric survey. The expanded version of ASKAP will detect 'baryon oscillations' at small spatial scales only.

project is entering a crucial phase of "system design" scheduled for the period 2008 - 2010. An essential question to be addressed is what combination of collecting area, angular resolution and bandwidth is required for performance of the key science projects. Unfortunately, current radio observations provide very weak guidance for these design studies, because almost nothing is known about the distribution of neutral hydrogen beyond the very local Universe. ASKAP will provide a very significant step forward in this respect.

\subsection{A Byproduct of HI surveys: OH Megamasers with ASKAP}

The wide field of view of ASKAP makes blind surveys for extragalactic $\mathrm{OH}$ megamasers a possibility. In fact blind surveys for $\mathrm{OH}$ megamasers represent a particularly exciting add-on to HI surveys at zero cost in observing time.

A rich series of transitions in $\mathrm{OH}, \mathrm{H}_{2} \mathrm{O}$ and methanol molecules can be driven into population inversions to generate powerful maser emission (Darling et al. 2003). Especially important for observations out to high redshifts are likely to be the 1665/1667 $\mathrm{MHz} \mathrm{OH}$ maser lines and 1612/1720 MHz conjugate lines (Darling 2004).

To conduct a successful search for $\mathrm{OH}$ megamasers, an appropriate channel width for detection is about $30 \mathrm{~km} \mathrm{~s}^{-1}$, or $0.17 \mathrm{MHz}$ at $\mathrm{z}=0$. For a 12-hour integration, we expect an rms noise level of $0.4 \mathrm{mJy}_{\text {beam }}{ }^{-1}$, or $2 \mathrm{mJy}$ at $5 \sigma$ significance. One can then 
expect to detect all $\mathrm{OH}$ gigamasers $\left(L_{\mathrm{OH}}>10^{4}\right)$ out to $\mathrm{z}=1$ and all $\mathrm{OH}$ megamasers with $L_{\mathrm{OH}}>10^{3}$ out to $\mathrm{z}=0.5\left(834\right.$ and $1112 \mathrm{MHz}$, respectively). $L_{\mathrm{OH}}=10^{3} \mathrm{~L}_{\odot}$ roughly represents the median luminosity of known $\mathrm{OH}$ megamasers. Based on the $\mathrm{OH}$ megamaser luminosity function, the expected number of $\mathrm{OH}$ megamasers detected at $0<z<0.5$ per 30 square degree region of the sky is conservatively of order 1.3.

Including an $\mathrm{OH}$ megamaser search in the planned $\mathrm{H}$ I search would not cost anything extra in terms of bandwidth or survey design. In particular, a bandwidth of $20 \mathrm{~km} \mathrm{~s}^{-1}$ as previously discussed for HI surveys would match requirements very well. The $\mathrm{OH}$ megamasers would essentially be free additional data products. A survey with $300 \mathrm{MHz}$ of instantaneous bandwidth from 1400 to $1100 \mathrm{MHz}$ would be an ideal $\mathrm{OH}$ megamaser search for this facility, and highly compatible with the H I survey. No other facility will be competitive in identifying new $\mathrm{OH}$ megamasers, particularly in a "blind" host-independent manner.

If the survey were to cover the entire southern sky, we might expect the detection of $\sim 10000$ new $\mathrm{OH}$ megamasers - just over 100 are known today. Such a windfall would be revolutionary in terms of enhancing numbers, pushing the detections to higher redshifts, and in particular studying the merging rate of galaxies (along with associated black hole feeding and merging and extreme bursts of star formation).

As a specific and exciting field of research offered by such objects, we note the recent measurement of Zeeman splitting in extragalactic $\mathrm{OH}$ megamasers by Robishaw et al (2008) using the Arecibo Radio Telescope. OH megamasers are typically found in ULIRGs (ultra-luminous infrared galaxies) so that these Zeeman observations provide the first magnetic field estimates in powerful starburst galaxies at significant redshifts. ARP220 and IIIZw35 were detected with good signal-to-noise ratio using the the full sensitivity of the Arecibo Telescope. Magnetic fields in $\mathrm{OH}$ masers now have been detected out to $\mathrm{z}=0.25$. Extended ASKAP has just enough sensitivity to do the Zeeman experiment, requiring a few days integration for the strongest $\mathrm{OH}$ megamasers.

To interpret the results, Robishaw et al (2008) had to use previous VLBI measurements to separate the different components of the velocity profile which correspond to different $\mathrm{OH}$ megamaser sites inside the starburst galaxy. The unique advantage of ASKAP for this research will be the VLBI baselines to the Australian east coast (see Chapter 6) which can separate the multiple components in the starburst galaxy. The $\mathrm{OH}$ megamaser spots will still be unresolved (P. Diamond, private communication), but only a single high-sensitivity baseline (e.g. ASKAP to Parkes) is needed to separate the maser spots in the Zeeman measurement, while smaller dishes can image the $\mathrm{OH}$ megamaser sites. 


\section{Continuum Science}

Lead authors: I. Feain, J. Wall

Contributing authors: C. Blake, R. Ekers, A. Hopkins, C. Jackson, R. Norris

\subsection{Summary}

Understanding the formation and evolution of galaxies and active galactic nuclei (AGN) as a function of cosmic time is one of the most intensely investigated issues in contemporary astronomy, and is a key science driver for next-generation telescopes such as SKA, ALMA and ELTs. Today's instruments already give profound insights into the galaxy population at high redshifts, and a number of current surveys (e.g. ATLAS, ELAIS, GOODS, COSMOS) are asking questions such as: When did most stars form? How do AGNs influence star and galaxy formation? What is the spatial distribution of evolved galaxies, starbursts, and AGNs at $0.5<z<3$ ? Are massive black holes a cause or a consequence of galaxy formation?

However, most of these surveys are primarily at optical and IR wavelengths, and can be significantly misled by dust extinction. Radio observations not only overcome dust extinction, but also provide data on AGN that are unavailable at other wavelengths. ASKAP will be able to determine how galaxies formed and evolved through cosmic time, by penetrating the heavy dust extinction which is found in AGN at all redshifts, and studying the star formation activity and AGN buried within.

\subsubsection{A Deep Southern Sky Survey}

ASKAP has the potential to have the enormous impact that the NRAO VLA Sky Survey (NVSS; Condon et al. 1998) had a decade ago, but at a factor of 50 better in sensitivity and 9 in angular resolution. This is particularly significant because of the growth in the number of ground-based telescopes (ALMA, VLT, Gemini, etc.) that will operate in the southern hemisphere. ASKAP will survey the entire southern sky to a limit currently being reached only in tiny parts of the sky (e.g. in the HDF-N and HDF-S deep fields). We will argue that these sensitivities can only be achieved with relatively high angular resolution (say $5^{\prime \prime}$ at $1400 \mathrm{MHz}$, requiring baselines to $\sim 8 \mathrm{~km}$ ).

Assuming the ASKAP strawman parameters, the entire southern sky can be surveyed to an rms noise limit of $10 \mu \mathrm{Jy} \mathrm{beam}^{-1}$ in one year. If we adopt an example synthesized beam of $5^{\prime \prime}$, the confusion level is well below the noise limit, and would broaden the observational distribution by less than $10 \%$ (J. Condon, private communication). Such resolution is important to achieve unambiguous cross-waveband identification. It is also important that the 'confusion limit' be far below the noise limit, because the confusion error is highly non-Gaussian with a long tail to the positive side.

This astonishing survey feat would provide a database of dramatic consequence for astrophysicists and cosmologists alike.

The following sections will describe a pick of the science programs that could be done with such a fast survey telescope. The $(T / N)^{2}$ dependence on both the point source and surface brightness sensitivity means that the extra dishes $(N)$ and lower system temperatures $(T)$ being proposed in the upgraded parameters would enable 
Table 3 Predicted numbers of sources per square degree at $1400 \mathrm{MHz}$ with a $10 \mu \mathrm{Jy}$ flux density limit, assuming standard cosmology. Table adapted from table 7 of Jackson (2005).

\begin{tabular}{lccc}
\hline Redshift & N(FRI) & N(FRII) & N(SF) \\
\hline $\mathrm{z}<1$ & 718 & 2 & 2556 \\
$\mathrm{z}<3$ & 1145 & 21 & 6740 \\
$\mathrm{z}<5$ & 1190 & 42 & 7584 \\
$\mathrm{z}>5$ & 17 & 13 & 0 \\
\hline
\end{tabular}

this same southern sky survey could be done in $1 / 5$ of the time, or only 2 months. To this end, we also include a few more ambitious science programs in this chapter that require the faster survey speeds offered by the upgraded ASKAP.

It is worth emphasising that at $5^{\prime \prime}$ resolution, incompleteness becomes significant as emission extended on scales larger than $5^{\prime \prime}$ is resolved (see the discussion regarding the choice of angular resolution for the NVSS in Condon et al. 1998). This is a doubleedged sword: higher angular resolution leads to a lower confusion limit (allowing a higher-sensitivity survey), but at the expense of completeness. However, this issue is likely to be less of a problem for the proposed ASKAP survey than for NVSS for two reasons. First, at the extremely faint flux densities of the proposed ASKAP survey, the source counts are dominated by distant star-forming galaxies with $\sim 1^{\prime \prime}$ angular sizes (see Table 3 and section 3.2 below), rather than the larger and more powerful radio galaxies which dominate the source counts in NVSS. Second, a continuum all-sky survey with short baselines will be available as a by-product of the proposed H I sky survey. While this compact continuum survey will be confusion-limited, the data can be sensibly combined with the longer-baseline $5^{\prime \prime}$ resolution southern sky survey to create a complete and high-resolution sky survey.

\subsection{Star Forming Galaxies across the Universe}

With a flux density limit of $5 \sigma=50 \mu \mathrm{Jy}_{\text {beam }}{ }^{-1}$, typical starburst galaxies, with star formation rates (SFRs) around $100 \mathrm{M}_{\odot} \mathrm{yr}^{-1}$, will be detectable to $z \approx 2$. The most extreme starbursts, with SFRs of a few $1000 \mathrm{M}_{\odot} \mathrm{yr}^{-1}$, will be visible well into the epoch of reionisation $(z \approx 6-10)$ if indeed they existed then. Ordinary disk galaxies like the Milky Way, with SFRs of only a few $\mathrm{M}_{\odot} \mathrm{yr}^{-1}$, will be visible to $z \approx 0.3$ (see Figure 11). A very deep sky survey utilising the $8 \mathrm{~km}$ baseline configuration, obtained in addition to an ultra-compact $\mathrm{H}$ i survey, will make the star-forming galaxy population accessible as never before. It will allow unprecedented exploration into how star formation in galaxies evolves with time, and how it depends on galaxy mass, galaxy environment, past star formation history, interaction/merger history, and more.

One of the most intriguing recent results in galaxy evolution is the mass dependence of galaxy star formation histories (Feulner et al. 2005; Juneau et al. 2005; Papovich et al. 2006). Evidence is growing that points towards a picture in which massive galaxies form their stars early and quickly, and "turn off" at redshifts around $z \approx 2-3$, while progressively lower mass galaxies come to dominate the cosmic star formation history at progressively lower redshifts. This is "cosmic downsizing", first described by Cowie et al (1996). The evidence for it comes not only from the mass-dependence but also from the impact of a galaxy's environment on its star formation rate (Lewis et al. 2002; Gomez et al. 2003), and its observed evolution (Poggianti et al. 2006). Recent results 


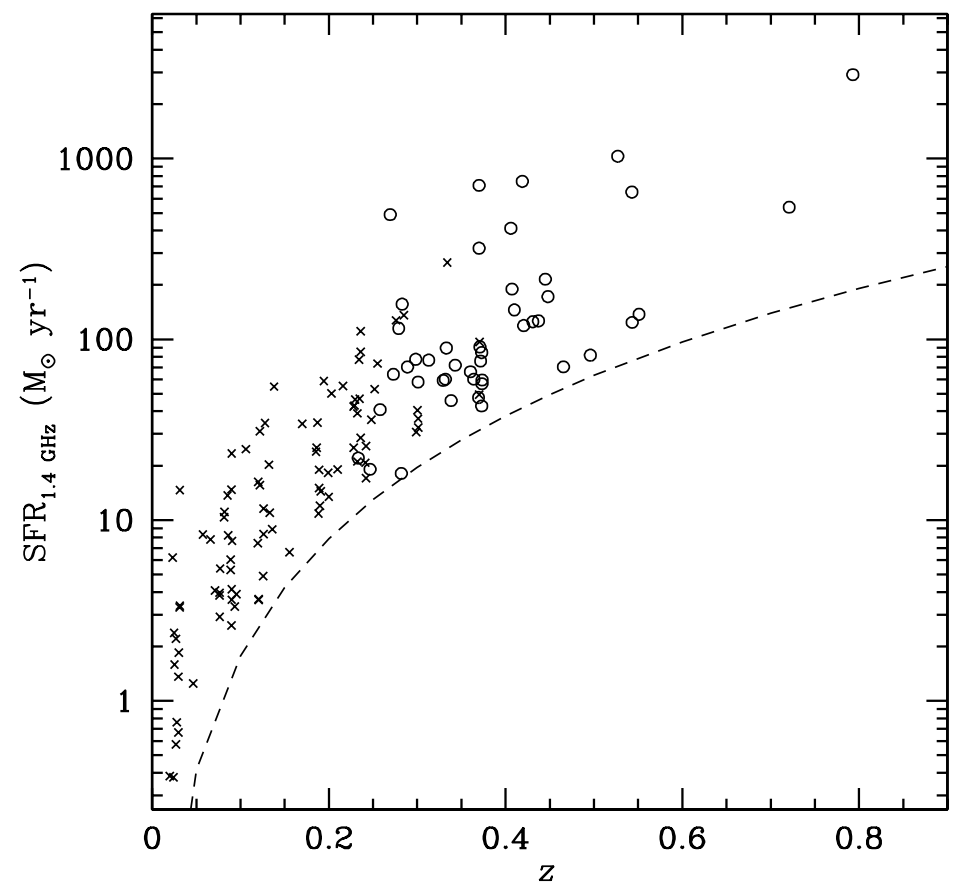

Fig. 11 SFRs for star-forming galaxies in the Phoenix Deep Survey, as estimated from their radio luminosity. The dashed line indicates the $60 \mu \mathrm{Jy}$ detection limit of the radio observations. Figure reproduced from Afonso et al. (2003).

by Zheng et al. (2007) question this picture, though, suggesting instead that the cosmic star formation history declines uniformly in galaxies of all masses at redshifts less than unity.

Resolving the star-formation question is fundamental for identifying how galaxies form and evolve through their star formation histories, the impact of their mass and their local environment, and the consequences on the resulting stellar populations and galaxy morphologies observed. This is only achievable with the large samples of strongly star-forming galaxies made accessible by deep continuum surveys over large areas such as those planned for ASKAP. The limiting factor affecting these studies will clearly be the resolution of ASKAP. Apparent galaxy sizes at radio wavelengths steadily decrease with decreasing flux density (Windhorst 2003). (To some extent this may reflect the intrinsically smaller sizes of high redshift galaxies in a hierarchically evolving universe.) The median angular size at $1 \mathrm{mJy}$ is about $2^{\prime \prime}$, and at $100 \mu \mathrm{Jy}$ is about $1^{\prime \prime}$. By $10 \mu \mathrm{Jy}$ the median angular size has dropped to about $0.4^{\prime \prime}$ (Windhorst et al. 1999; Windhorst 2003). With the suggested $5^{\prime \prime}$ resolution of ASKAP, instrumental confusion will be the limiting factor governing the sensitivity of continuum surveys, and this will remain the case until baselines spanning several tens up to hundreds of kilometres can be incorporated in the SKA. To mitigate the resolution limitations, it 
is highly desirable to match the proposed ASKAP surveys with complementary deep optical/near-infrared surveys, having much finer angular resolution. Here the southern sky surveys of SkyMapper (Keller et al. 2007) will be of vital importance in the first instance.

\subsection{The Star Formation / AGN Fraction}

Another important factor to consider is distinguishing the star forming population from the AGN population. Below 1 mJy starburst galaxies start to become a major component of the $1.4 \mathrm{GHz}$ source counts, dominating below $0.5 \mathrm{mJy}$ or so (Hopkins et al. 2000; Jackson 2005). Recent measurements, though, suggest that there may still be a significant proportion of low-luminosity AGN (Simpson et al. 2006) even at these levels. By $0.1 \mathrm{mJy}$, existing AGN evolutionary models imply that starburst galaxies should be dominant, although normal star forming galaxies are not expected to dominate the counts until levels below $\sim 1 \mu \mathrm{Jy}$ are reached (Windhorst et al. 1999; Hopkins et al. 2000).

Traditionally separate fields of astrophysical research, it is now becoming clear that AGN and star formation activity are intimately related. The question is how does galaxy formation influence the growth of the central black hole, or vice-versa? Many galaxy formation models now invoke feedback by supernovae, starbursts or AGN, while many others are now emphasising the importance of feedback due to powerful radio jets, which either push back and heat the infalling gas, reducing or even stopping the cooling flows building up the galaxy or shock heat and collapse the gas clouds and induce star-formation. There is no doubt that some feedback (positive or negative) between the AGN and the growth of the host galaxy is occurring, but it is hotly debated as to the nature of the feedback. A detailed study of the AGN and star formation activity in high redshift galaxies will help answer some questions. The sheer number of sources down to faint flux density limits that will be found with ASKAP will go a long way in this regard. Starforming galaxies could be identified in the samples using a variety of methods:

1. Analysis of optical and near-IR spectral energy distributions (SEDs).

2. Comparison of radio and far infra-red (FIR) flux densities and exploiting the radioFIR correlation for star forming galaxies; see section 3.6.

3. A wide-field VLBI program between ASKAP and telescopes on the east coast (such as the Parkes $64 \mathrm{~m}$ ) which would allow the fraction of radio emission in galaxies associated with a central, compact object (AGN) to be determined. We already know that AGN with starforming host galaxies exist (see Figure 12); the question is how significant is this class of objects with respect to the overall population of radio sources across cosmic time?

\subsection{Radio Galaxy Evolution across Cosmic Time}

There is an inevitable degeneracy between luminosity and redshift inherent in fluxlimited samples of classical (active) radio galaxies (Figure 13). This arises because only 

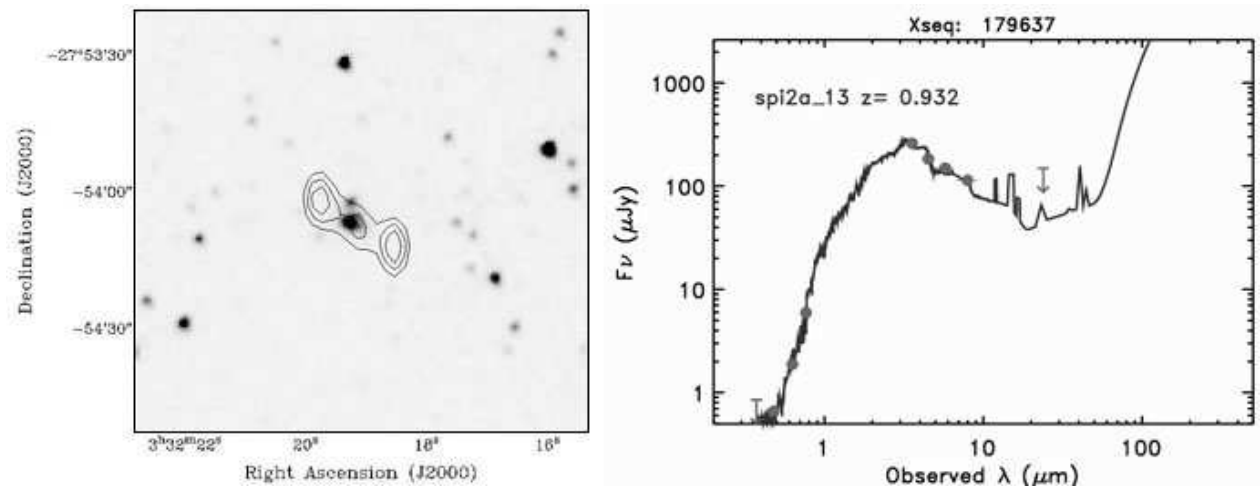

Fig. 12 The composite source S425. The left image shows radio contours superimposed on a $3.6 \mu \mathrm{m}$ SWIRE image, and the right image shows the SED. This source has the radio morphology of an AGN, but the SED of a star-forming galaxy.

the most powerful sources are detected at the largest redshifts. In order to explore the physics of radio galaxies, and especially their evolution, large samples of radio galaxies of comparable luminosity across cosmic time are required. Most attempts to investigate changes in radio galaxy properties as a function of redshift have been severely limited by the inability to separate luminosity-dependent effects from true cosmological evolution. With the relatively slow survey speeds of existing radio telescopes, this

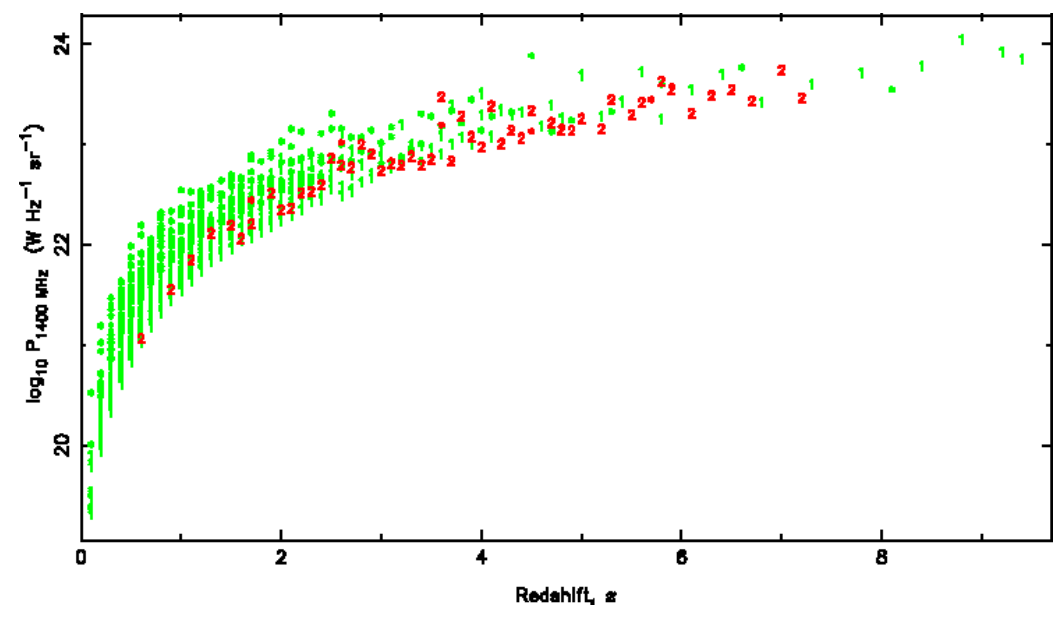

Fig. 13 Power-redshift distribution, $\mathrm{P}-\mathrm{z}$, for FRI and FRII sources at $1400 \mathrm{MHz}$ for a flux density limit of $10 \mu \mathrm{Jy}$ from Jackson (2005). Key: FRI radio galaxies (1), FRII radio galaxies $(2)$, beamed sources $\left(^{*}\right)$.

degeneracy has in the past been broken by combining samples of radio sources selected at similar observing frequencies, but with different flux density limits (Blundell et al. 1999). However, we are still unable to say much about the global population of radio 
galaxies at redshifts above about 0.5 because our faint source counts are so limited. With current radio sky surveys we are simply unable to reach the luminosities required to find, if they exist, FRI (Fanaroff and Riley 1974) radio galaxies at cosmologically significant distances. An FRI radio galaxy with a typical luminosity at $1400 \mathrm{MHz}$ of $10^{24} \mathrm{~W} \mathrm{~Hz}^{-1} \mathrm{sr}^{-1}$ has an observed flux density of $2 \mathrm{mJy}$ at $z \sim 1$ and $50 \mu \mathrm{Jy}$ at $z \sim 4$ for sources with a radio spectral index of -0.8 . These low flux densities demonstrate why this experiment has not been possible in the past and why deep, large-FoV radio continuum surveys are essential if we are to understand active radio galaxy evolution in any detail. FRI type sources make up the vast majority of local radio sources and yet we know very little about their existence at higher redshifts; high redshift FRIs may even contribute up to $2 / 3$ of the total source counts at sub-mJy levels (Padovani et al. 2007). The fact that this is at variance with some current models of space distribution- Table 3 suggests that we might expect them to contribute 15 per cent at $10 \mu \mathrm{Jy}$ - merely emphasizes the impact which ASKAP type surveys will have on the experiment. ASKAP will detect radio sources down to $5 \sigma=50 \mu \mathrm{Jy}_{\text {beam }}{ }^{-1}$ with a dynamic range of $10^{5}$ and essentially fill the luminosity-redshift plane (see Figure 13); assuming, of course, we obtain redshifts for the sources in the sample. The results of the proposed ASKAP deep southern-sky survey contribute a wealth of knowledge in this field of research a field which has stalled, waiting for the advent of big survey telescopes. (Acquiring redshifts has been the severe limitation throughout the long history of this program; but large sky surveys and in particular the prospect of the Large Synoptic Telescope will - by the advent of ASKAP- alleviate the problem.) Precise definition of the luminosity functions of FRI and FRII radio sources as a function of redshift is essential for us to understand the formation and evolution of active radio galaxies. This science should not be underestimated: radio galaxies represent the most massive galaxies at any redshift up to $z=5.2$ and therefore represent our best chance to understand the formation and evolution of massive galaxies and their central supermassive black holes. In addition, they are likely also to provide the dominant source of energy in the universe, and possibly even provide the dominant source of magnetic fields. Finally as we describe in the following section, AGN now appear to represent a crucial feedback phase in galaxy evolution, without which our Universe of galaxies would bear little resemblance to the one we inhabit.

\subsubsection{Space Density Evolution of Radio Galaxies}

The space density of radio active objects is handed over at $z<1$ from the highluminosity FRII radio galaxies of the 'evolution bulge' to the mildy evolving and lowerluminosity radio galaxies which dominate the space density at local epochs (Longair 1966, Dunlop and Peacock 1990, Willott et al 2001). The break in luminosity for this space density evolution is well above the FRI/II break so that the mildy-evolving luminosity bin includes sources with both FRI and FRII morphologies (Willott et al. 2001). Thus, while it is well established that the most powerful FRII sources must evolve more rapidly than their lower-luminosity counterparts, little is known about the evolution of FRIs because we do not reach the FRI/FRII luminosity threshold beyond $z \sim 0.5$.

In addition, at $z>>1$ some radio galaxies have morphologies strikingly different to the canonical FRI and FRII morphologies we are used to observing at $z<1$. These galaxies are characterised by the alignment of the radio and optical axes (van Breugel 
et al. 1999) and somewhat irregular morphologies which are not clearly FRI or II. How these sources evolve is also an open and extremely interesting question.

What causes the luminosity-dependent space density evolution of FRII radio galaxies? Is there a similar evolution in the space density of FRIs and of the high-redshift radio galaxies not easily categorised into FRI or FRII morphology? What feedback mechanisms are at work effecting, for example, the changeovers in the AGN activity from large numbers of high-luminosity sources at higher redshifts to the dominance of lower luminosity sources in the local universe? There is no doubt a rich rich range of phenomena at play, from development of black holes and their accretion disks, the relation of this to overall galaxy mass, to star formation, agglomeration and galaxy construction, to trigger mechanisms for radio emission, to different type of accretion engine perhaps responsible for the differentiation in morphology between FRI and FRII, to either positive or negative AGN feedback mechanisms that could trigger or quench, respectively, the types of radio emission and star formation.

Positive feedback mechanisms have been identified for some time through the apparent coincidence between radio and optical axes of high-redshift radio galaxies - the alignment effect (McCarthy et al 1987, Chambers et al 1987). The effect is attributed to jet-induced star formation; radio AGN jets propagate into overdensities and shockinduce massive star formation (Bicknell et al. 2000). The clumpy nature of radio-galaxy hosts at high redshifts, with the clouds of gas and dust aligned with radio emission (Klamer et al. 2004), suggests a picture in which black holes and jets are in place before host galaxies are fully formed. The black hole - jet activity may even play a role in deciding the morphology of the host galaxy.

However, it is negative feedback mechanisms that have brought AGNs to the forefront in modern scenarios of galaxy formation. With regard to the largest galaxies, several authors (Sanders et al. 1988, Granato et al. 2001, Archibald et al. 2002, Stevens et al. 2005, Di Matteo, Springel and Hernquist 2005) have suggested a connection between the formation of powerful QSOs and ULIRGs (Ultra-Luminous Infra-Red Galaxies), or their high-redshift counterparts, the sub-millimetre galaxies. A picture has emerged of an evolutionary sequence in which the forming galaxy is initially far-infrared luminous but X-ray weak, similar to the sources discovered as sub-millimetre galaxies. As the black hole and spheroid grow with time, the central QSO becomes powerful enough to terminate the star formation and eject the bulk of the fuel supply. This feedback transition is followed by a period of unobscured QSO activity, subsequently declining to leave a quiescent spheroidal galaxy. Recently it has been shown that the space densities of QSOs and submillimetre galaxies show remarkable similarity in epochal behaviour (Wall et al. 2005, 2008), providing significant support to such a scenario. Nevertheless, direct evidence remains elusive. For example, detailed CO observations with ATCA by Papadopoulous et al. (2008) indicate that for the so-called 'transition' quasar HE04502958 , the CO originates from a disturbed and gas-rich companion interacting with the presumably gas-poor QSO. They suggest that other such candidates may simply be gas-rich/gas-poor galaxy interactions triggering QSOs in the latter.

For galaxies large and small, the only formation scenario fitting the bulk of observational data - luminosities and the structure of the cosmic web in particular - has proved to be hierarchical buildup in a $\Lambda \mathrm{CDM}$ cosmology. Using huge and sophisticated numerical simulations, semi-analytic modelling of this process has become a major industry (e.g. Springel et al. 2005). There are brilliant successes, but until recently, some stark failures (see Bower et al. 2006). The most glaring of these is that hierarchical buildup implies current-epoch galaxies should be the largest, youngest and bluest, 
with star-forming maximized. Instead they are predominantly older, with the bulk of star formation carried by galaxies significantly smaller than at earlier epochs - cosmic downsizing, as first described by Cowie et al. (1996). AGNs have been invoked to rescue the situation. New simulations (Croton et al. 2006, Bower et al. 2006) incorporate the energy injected by central supermassive black holes (e.g. Silk and Rees 1998, Quilis et al 2001, Granato et al 2001, Brueggen \& Kaiser 2002) to quench star formation and break the hierarchical buildup. Indeed Silk \& Rees (1998), Rawlings \& Jarvis (2004), and Granato et al. (2004) demonstrate effectively that AGN energy input cannot fail to have a significant effect on galaxy formation. The energy of radio AGN fired into the X-ray-bright Inter-Cluster Medium shows up observationally as jet-driven shocks and bubbles in the ICM of nearby galaxy clusters (Blanton et al. 2004, Fabian et al. 2005).

But which feedback mechanism dominated when? Was it positive or negative? And what was the precise mechanism? (Several are on offer for both positive and negative feedback.) Breakthrough data from an ASKAP survey will provide giant steps towards understanding the majority of these vital galaxy-formation issues. The large samples of sources it will supply at different flux-density levels, coupled with optical data from e.g. PanStarrs and the LSST, will generate samples from which unambiguous luminosities can be determined. ASKAP will have the sensitivity to detect FRIs out to $z \sim 4$ (see Section 3.4). In order to determine a source morphology, we assume that at least 8 synthesised beams are required and that each beam contains at least $5 \sigma$. This means that morphologies can be measured for sources with total flux densities larger than $0.4 \mathrm{mJy}$, corresponding to typical FRIs at a threshold redshift of $z \sim 1.7$ with linear sizes $\gtrsim 100 \mathrm{kpc}$. Subsamples can be mapped at higher resolution with telescopes such as ATCA. From both directions we will be provided with a rich set of samples from which population synthesis will yield epoch dependences and birth functions for FRI, FRII and high-redshift radio galaxies. True integration of radio AGN data into galaxy formation can then be contemplated.

\subsubsection{Unified Models}

Unified models describe how 'compact', and 'flat spectrum' sources, predominantly identified with radio QSOs and BL Lac Objects, are the same objects as 'steep spectrum', 'double-lobed' sources, predominantly identified with radio galaxies.

Unification schemes arose in the early 1980s following the VLBI discovery of superluminal motion in the 'blobs' of compact emission emerging from QSO cores. If the blobs emerge at relativistic speeds, then alignment of the ejection axis with the observer line of sight provided an explanation via Doppler effects; and this also explained the one-sidedness of QSO jets, the anti-jets suffering from Doppler de-boosting. It only required some way of hiding the QSO, the direct view of the accretion disk system, from the observer when viewed side-on. The dusty torus (Antonucci \& Miller 1985) served the purpose. Many subsequent observations, such as the detection of the faint double-lobe structure about compact QSOs, served to confirm the general view - radio galaxies, quasars and BL Lacs are the same objects, with angle of ejection axis to the line-of-sight as the critical parameter.

The description of a 'dual population' unified model (Jackson \& Wall 1999) took this a stage further, the dual populations being FRIs with BL Lac objects as their beamed counterparts, and FRIIs with both BL Lacs and QSOs as counterparts. In this investigation, the bulk data from radio surveys were used to estimate beaming 
parameters and thus to get an unbiased view of the ejection physics for radio QSOs and BLLac objects. The results showed that such a dual population model was not only supported by survey data (source counts, luminosity distributions) but also produced beaming parameters in reasonable agreement with those determined by arduous individual VLBI monitoring of individual objects.

However, this was a bare-bones scheme, in the sense that only the best single value, non redshift dependent parameters were described, such as beaming Lorentz factors and the proportion of emission beamed. But this is a poor description of the extensive range of physics involved: how the Lorentz values are actually distributed and how beaming depends on luminosity and on cosmic epoch were not investigated. Significant refinements, in particular the examination of the 'receding torus' model, were introduced by Grimes et al. (2004). Nevertheless, ideally we require a 100-fold increase in sample sizes to make significant progress. ASKAP would provide an essential step - sizeable samples at an impressive range of flux-density levels - towards critical examination or perhaps successful development of this model. Complete high-resolution images to determine morphologies and optical data to determine identifications and redshifts are also required. Deduction from such a powerful data base is a relatively painless way of discovering the physics at the unresolved and unresolvable hearts of AGN.

\subsubsection{Searches for the Highest Redshift Radio Galaxies}

Statistically large samples of high-redshift radio galaxies (HzRGs) are slowly being accumulated, with more than 30 known to date above $z=3$. These samples are assembled by data-mining existing radio sky surveys, and selecting sources with unusually steep radio spectral indices, taking advantage of the correlation between increasing redshift and steepening radio spectral index ${ }^{2}$.

The need to data-mine wide-area radio sky surveys to define sufficiently large steep spectrum samples for optical follow-up is obvious: HzRGs are extremely rare. Between $3<z<4$, the minimum space density of powerful $\left(>10^{26} \mathrm{~W} \mathrm{~Hz}^{-1} \mathrm{sr}^{-1}\right)$ radio galaxies is $1.2 \pm 0.6 \times 10^{-9} \mathrm{Mpc}^{-3}$ and their minimum surface density is $63 \pm 29 \mathrm{sr}^{-1}$ (De Breuck et al. 2006). By virtue of the flux density limits of existing sky surveys (several mJy at $1.4 \mathrm{GHz}$ ), the HzRGs detected to date are all amongst the most powerful radio galaxies (FRII class) that we observe at lower redshifts. For example, the HzRGs selected (De Breuck et al. 2006) by cross-matching the $1400 \mathrm{MHz}$ NVSS with the $843 \mathrm{MHz}$ SUMSS (Mauch et al. 2003), have radio luminosities of order $10^{26-27} \mathrm{~W} \mathrm{~Hz}^{-1} \mathrm{sr}^{-1}$, thus probing just the tip of the iceberg. Cygnus $\mathrm{A}$, the most powerful radio galaxy in the nearby universe $(z=0.056)$ has a $1400 \mathrm{MHz}$ radio luminosity of $\sim 10^{27} \mathrm{~W} \mathrm{~Hz}^{-1} \mathrm{sr}^{-1}$. To date, little to nothing is known about the lower luminosity (e.g. FRI) population of sources at high redshift. This is a major shortfall with current studies of high redshift radio source populations; see previous section.

Two deep surveys, done at widely separated frequencies, would be a huge improvement on existing capabilities to find HzRGs using the steep-spectrum selection technique. The surveys could be done at, for example, $700 \mathrm{MHz}$ and at $1800 \mathrm{MHz}$; but the confusion limit will be significantly higher at $700 \mathrm{MHz}$. Instead, surveys done at

2 Although this technique increases the likelihood of finding radio galaxies at high redshift, it severely biases the resultant samples to only those with unusually steep spectra. As a result, we currently know very little any population of normal-spectrum radio galaxies at high redshift. 
$1400 \mathrm{MHz}$ and $2700 \mathrm{MHz}$ would be far more valuable in terms of reaching FR I-class radio galaxies at high-redshift. This program would benefit hugely from the $1 / 5$ survey speed offered by the upgraded ASKAP parameters because the two southern sky surveys will take only 4 months instead of 2 years. Most importantly, ASKAP will reach sensitivities a factor of 300 times fainter than existing steep-spectrum samples ${ }^{3}$, thereby sensitive to lower-luminosity radio galaxies. Another benefit of the ASKAP steep-spectrum sample over current samples is that a single telescope producing two sky surveys at different frequencies removes systematic errors inherent in cross-matching surveys made with different telescopes, sometimes with vastly different angular resolutions. The fast survey speeds offered by ASKAP also reduce the number of spurious spectral indices caused by source variability on time scales of the two surveys. Further compression of the time frame could be achieved by doing the two surveys in parallel, rather than contiguously.

Once the steep-spectrum samples have been selected, optical (from SkyMapper) and then deep near-IR imaging would be required to identify the host galaxies. Spectroscopy of the faintest near-IR hosts would be required to secure their redshifts.

To avoid source confusion and to enable cross-waveband identifications, this program requires baselines out to $8 \mathrm{~km}$ (angular resolution of $5^{\prime \prime}$ at $1400 \mathrm{MHz}$ ). The spatial resolution offered with an $8 \mathrm{~km}$ baseline would not allow us to image the radio sources (most HzRGs have angular sizes $<5^{\prime \prime}$ ) but if the visibility data were retained we would be able to measure source sizes to better than $1^{\prime \prime}$. ASKAP will certainly archive calibrated visibility data for all continuum observations. There is a strong negative correlation between the linear size of radio sources and redshift, but presently we cannot say much more about this because we are constrained by Malmquist bias. ASKAP will produce sufficient numbers of HzRGs at low enough radio luminosities that we will be able to explore this linear size - redshift correlation and separate out radio-luminosity dependent effects from cosmological evolution. We will be able to do this with the archived visibility data, which will enable measurement of radio source sizes down to $1^{\prime \prime}$ or better for the higher signal-to-noise sources.

\subsubsection{Radio Spectral Energy Distributions}

The HzRGs known to date (biased heavily by the steep-spectrum selection technique) have radio spectral energy distributions (SEDs) very well characterised by single power laws (Klamer et al. 2006). This appears in stark contrast to the majority of known radio galaxies that have curved SEDs, steepening toward higher frequencies. However, aside from a handful of studies, very little is known about the global/statistical properties of radio galaxy SEDs. ASKAP can contribute enormously in this regard. One thing it will do very well is to produce uniform sky surveys at a number of frequencies across its available band, each with well-defined and uniform systematics, and accurate flux densities. This will allow an investigation into the shapes of radio SEDs as a function of various radio-source parameters (linear size, redshift, luminosity, average spectral index). We would therefore advocate as large a frequency coverage as possible for ASKAP; but even 6 surveys, each separated by $300 \mathrm{MHz}$ in frequency between 700 and $2700 \mathrm{MHz}$, would open up a new region of radio-source parameter space about which

3 The SUMSS-NVSS USS sample was selected with a $15 \mathrm{mJy}$ flux density limit imposed at $843 \mathrm{MHz}$. 
we currently know little. Such surveys would likewise contribute immeasurably to the type of population studies and space evolution discussed in the previous section.

\subsection{A Low Surface Brightness Survey}

\subsubsection{Low Surface Brightness Galaxies}

The NVSS has a $5 \sigma$ surface brightness sensitivity of $0.7 \mathrm{~K}\left(4.5 \mathrm{mJy} \operatorname{arcmin}^{-2}\right.$ within the $45^{\prime \prime}$ synthesised beam). An ASKAP survey with a compact configuration will be sensitive to sources with up to 100 times lower surface brightnesses. At these low surface brightness levels, ASKAP will be sensitive to the diffuse emission from, for example, dying radio galaxies (Murgia et al. 2005) cluster haloes, relics and ghosts (e.g. Feretti 2002, Enßlin 1999, Enßlin \& Gopal-Krishna 2001), 'fat double' FRI tails and plumes (Subrahmanyan et al. 2006), and perhaps even the Thomson scattered electrons off Dark Matter (see Section 3.5.2). In addition, whilst not quite as good as the continuous $u v$-coverage offered by the SKAMP survey, for example, a compact (baselines out to $1 \mathrm{~km}$ ) ASKAP survey will be far superior to the NVSS in terms of sensitivity to large scale emission: in the snapshot mode which the NVSS was produced, the VLA is sensitive to largest angular sizes of $8^{\prime}$ whereas the ASKAP survey will be sensitive to largest angular scales of about $50^{\prime}$. A survey to find low surface-brightness sources could be done as follows:

1. Complete a deep survey to $\sigma_{r m s}=10 \mu \mathrm{Jy}_{\text {beam }}{ }^{-1}$ with baselines out to $8 \mathrm{~km}$. Proper source counts can then be made and a catalogue of sources on the sky will be derived. Ideally, this survey would only take 2 months by utilising the upgraded ASKAP parameters.

2. Complete a second continuum survey in the $\mathrm{H}$ I-optimised compact configuration (which we assume to be $1 \mathrm{~km}$; angular resolution $40^{\prime \prime}$ ) down to the same limit as above. This survey would be very confused but this does not matter at all for the science proposed.

3. The $8 \mathrm{~km}$ - configuration survey would then be smoothed to the resolution of the $\mathrm{H}$ I-optimised survey and subtracted, leaving only the sources which have surface brightnesses below the limit of the higher-resolution survey.

Sources whose structure is dominated by large scale emission will be left behind and we will, for the first time, be able to probe a parameter space that no past or existing radio sky survey has been able to access. Dead radio galaxies? Diffuse cluster halos and relics? Diffuse halos containing the missing Dark Matter (see section 3.5.2)? Who knows what else lurks below the current sensitivities of today's surveys.

\subsubsection{Thomson Scattering off Dark Matter}

A plausible repository for the $90 \%$ of missing baryons (Dark Matter) is in a diffuse intergalactic medium (IGM). Gunn \& Peterson (1965) showed that any such IGM must be ionised. One way to test for its presence is to search for halos around powerful highredshift radio galaxies caused by radio emission that has been Thomson-scattered by 
the ionised IGM (Sholomitskii \& Yaskovitch 1990; Geller et al. 2000). The brightness of the halo relative to the central source is a measure of the density of the IGM and thus a direct measure of $\Omega_{\mathrm{IGM}}$. Heroic efforts with existing telescopes to do this have resulted in $2 \sigma$ detections of a non-zero $\Omega_{\mathrm{IGM}}$ - providing plausibility of concept rather than any detection of the diffuse IGM. Halos are expected to be $1 / 3$ tangentially polarised with halo sizes decreasing in surface brightness as $1 / \mathrm{r}$, limited only by the age of the central radio emitting source and the speed of light. Therefore, for a QSO emission age of $10^{8}$ years at $z=3$, halos in principle could extend out to $1^{\circ}$. In addition, if the radio emission is intermittent then the halo would show a ring-like pattern.

The crucial parameter for an electron scattering experiment is sensitivity to low surface brightness structure, high dynamic range $\left(10^{5}\right)$ and good polarisation purity, since searches for halos should be done through their polarised signature to reduce confusion. The experiment requires surveys with both high and low spatial resolutions in order to properly remove (subtract) the bright confusing (point) sources from the field to be left with the diffuse, large-scale faint halos. The high dynamic range, polarisation purity and clean, uniform visibility data that is expected from ASKAP makes it an ideal telescope to do this experiment.

\subsection{The Radio-FIR Correlation}

One of the tightest correlations in extragalactic astronomy exists between the radio continuum (RC) and far-infrared (FIR) emission from low-redshift non-AGN galaxies (Condon 1992), even if the actual mechanisms driving the correlation are not absolutely clear. In order to establish the mechanism(s), one aspect is clearly necessary, namely to establish the flux level, the redshift, and thus the luminosity range to which this correlation continues to hold. Appleton et al. (2004) have demonstrated a good correlation between the $24 \mu \mathrm{m}$ and $20 \mathrm{~cm}$ flux densities for objects with a median $z=0.3$ extending out to $\mathrm{z} \sim 1$, and Beswick et al. (2006) showed that the luminosities of all identified $24 \mu \mathrm{m}$ sources in their extremely deep radio field still follow the correlation for sources with $24 \mu \mathrm{m}$ flux densities $\geq 80 \mu \mathrm{Jy}$. Exploration of the radio-FIR correlation at even fainter levels can only be obtained at present by the technique of stacking, and Boyle et al. (2007) have stacked ATLAS (Norris et al. 2006) radio data to show that, indeed, the radio-FIR correlation extends down to $\mu \mathrm{Jy}$ levels.

Figure 14 shows all ATLAS sources, in both the CDFS and ELAIS-S1 fields, which have measured $20 \mathrm{~cm}$ and $24 \mu \mathrm{m}$ flux densities. These sources include both star-forming galaxies, which presumably follow the radio-FIR correlation, and AGN, which are expected to lie above it. As a result, a lower bound is clearly visible, presumably corresponding to the radio-FIR correlation. Also shown are the stacked data from Boyle et al. (2007). Although the stacked data roughly follow the correlation, it is clear from Figure 14 that their extrapolation lies below the lower bound to the data. This is probably caused by a systematic error in the stacking procedure: however it is possible that the weak radio sources represent a different population of objects, or objects with an intrinsically different value of the FIR - radio continuum correlation coefficient (at $24 \mu \mathrm{m}$ ), from objects seen in the local Universe. The ASKAP survey data will help to resolve the issue, without stacking down to $50 \mu \mathrm{Jy}$, and with stacking down to still lower flux densities. 


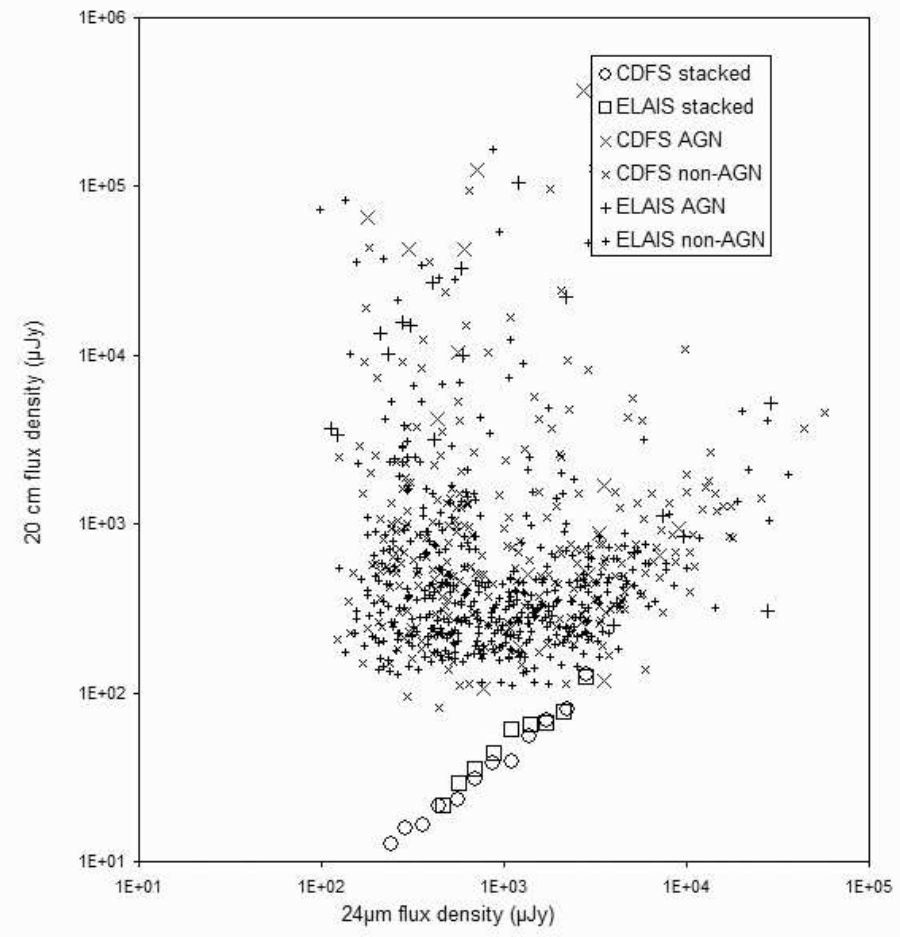

Fig. 14 The observed flux densities of all detected sources in the two ATLAS fields, together with the stacked data from Boyle et al. (2007). The AGN classification is determined by morphology or spectroscopic classification, while non-AGN means that the source has not been classified as an AGN, but may still be either an AGN or star-forming galaxy.

\subsection{Large-Scale Structure}

Radio AGN surveys provide a unique view of large-scale structure, unmatchable in depth, width and uniformity. Blake \& Wall (2002a; 2002b) determined how the largescale structure information locked up in major radio sky surveys can be revealed. In particular radio galaxies were shown to have (a) a two-point correlation function power-law slope of index -0.8 , in agreement with the slope found for optically-selected galaxies; and (b) stronger clustering than 'normal' galaxies, with a correlation scale length of $r_{0} \sim 7-10 \mathrm{Mpc}$. A survey with ASKAP will have the resolution and sensitivity to do far more:

1. Because ASKAP will reach below the $1 \mathrm{mJy}$ level, for the first time it will be possible to measure the correlation functions for the different populations represented at this level. How similar are the cosmic webs of AGNs and star-formers? Does this give us some further indication of the relation between these types of galaxy?

2. Uniformity and resolution will enable the surface analyses to be carried out for several populations. Populations such as FRI and FRII galaxies, radio quasars and BL Lac objects are different parts of the unification paradigm; see Section 3.3. Their cosmic 
web parameters (in similar redshift bands) should match identically if unification holds. This is a critical test of unification.
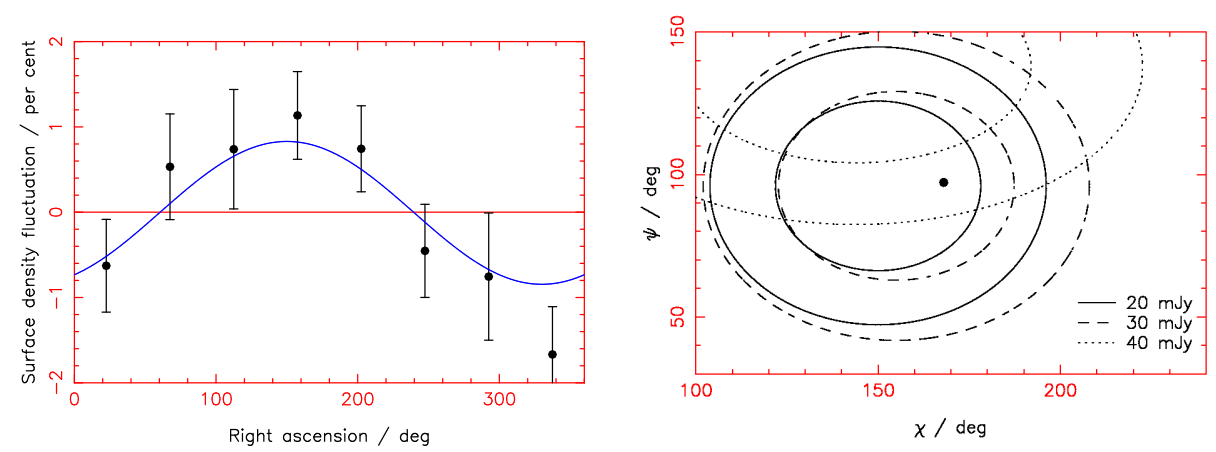

Fig. 15 Detection of the dipole anisotropy in the NVSS survey, as described by Blake \& Wall (2002c). Left panel: Amplitude of the anisotropy, agreeing in scale and phase with the predicted sine wave. Right panel : the direction, as calculated at different flux density levels; the large dot is the position of the maximum of the CMB dipole. Only sources in NVSS above $20 \mathrm{mJy}$ were used, because of the systematic calibration effects which affect the survey at lower levels.

3. By removing all nearby galaxies from the NVSS survey, Blake \& Wall (2002c) were able to demonstrate that a dipole anisotropy is present in the NVSS radio galaxies, agreeing in amplitude and direction with the dipole seen in the Cosmic Microwave Background. The results are shown in Fig. 15. The dipole has the predicted amplitude, and its direction, crudely measured, agrees with that of the CMB. This is the first time that the dipole anisotropy has been detected in discrete galaxies. The Universe is uniform at a redshift of $\sim 1.2$, as it is at a redshift of $\sim 1100$. The deep ASKAP survey would provide a directional measurement of far greater precision. Moreover it will permit tracing of the onset of uniformity; we shall be able to track the dipole as a function of epoch until it is overwhelmed by present-day large scale structure, adding an important constraint to structure development and galaxy formation.

\subsection{The Integrated Sachs-Wolf Effect}

ASKAP may answer directly the question of whether Dark Energy exists at $z \sim 1$, and if so on what scale. The existence of Dark Energy is one of the most profound puzzles in cosmology. We believe in it indirectly: it is implied by the supernovae Type Ia Hubble relation; and it is implied by the combined inference of flat geometry $\left(\Omega_{\mathrm{tot}}=1\right)$ from CMB measurements and low mass density $\left(\Omega_{\text {mass }} \sim 0.3\right)$ from large-scale structure measurements. A unique independent test of its existence is a correlation between $\mathrm{CMB}$ fluctuations and low-redshift large-scale structure (Crittenden \& Turok 1996), the 'latetime Integrated Sachs-Wolfe (ISW) effect'. Such a correlation would only be seen if the CMB photons have been redshifted by the low-redshift structure as it collapses, and only occurs if the current expansion of the Universe is NOT matter dominated.

It may be possible to detect this so called ISW effect by cross-correlating fluctuations detected in the $\mathrm{CMB}$ with large scale fluctuations in the mass distribution at 
$z \sim 1$ as probed by radio galaxies. Cross-correlation of the NVSS radio galaxies with the CMB anisotropies gave a tentative detection of the ISW effect, but at significance of only $2 \sigma$ (Boughn \& Crittenden 2004). A more recent analysis (Pietrobon et al. 2006) used WMAP3 data and powerful statistical techniques to exclude the null hypothesis at 99.7 per cent. However an extremely deep survey with ASKAP can provide a far more stringent examination; this test has a much lower confusion limit than the traditional limit for source counts, because we are looking for a statistical result on size scales of order the scales of the CMB anisotropies. Moreover, the deep ASKAP survey provides the ability to track the effect with redshift: if we do so, we have an effective probe of $\mathrm{w}(\mathrm{z})$, the equation of state of the Dark Energy as a function of epoch. This can be done through testing the correlation in different shells of relatively low $(z<1)$ redshifts. Finding parameters of the Dark Energy is perhaps the hottest topic in cosmology at present; and the deep ASKAP survey can provide vital constraints on them.

\subsection{A Weak Lensing Demonstrator}

Future radio telescopes, culminating in the SKA, can potentially become the world's leading facilities for performing cosmological surveys. In particular, the web of cosmic structure imprints a coherent shape distortion in the distribution of distant galaxies. This weak gravitational lensing is probably the most powerful probe of the characteristics of Dark Energy and Dark Matter. The SKA continuum survey can be used to construct such a lensing map featuring excellent image quality, a high source density to limit statistical noise, and a wide survey area to reduce cosmic variance. We note two key advantages compared to optical imaging surveys. First, the point-spread function of a radio telescope is well-determined and stable (being simply derived from the interferometer baseline distribution). Secondly, distance measurements in optical imaging surveys are derived from photometric redshifts, which suffer from systematic uncertainties, whereas the $\mathrm{H}$ I survey provides precise redshifts for a subset of galaxies at $z<1.5$.

ASKAP has insufficient collecting area and resolution to perform the weak-lensing experiment. However, it will pave the way for the SKA surveys by resolving key issues of survey design and analysis. This requires an ASKAP deep survey with at least $5^{\prime \prime}$ resolution in order to avoid confusion down to $\sigma=10 \mu \mathrm{Jy} \mathrm{beam}^{-1}$, and in order to map the angular sizes of the faint radio population down to $1^{\prime \prime}$. ASKAP will contribute by refining our knowledge in the following ways:

1. Measurement of the faint radio source counts over large areas. Future lensing surveys in radio wavebands will target star-forming disk galaxies, owing to the complex morphologies and low angular density of powerful FRI and FRII radio galaxies (see Table 3). The number density of these disk galaxies determines the future accuracy of Dark Energy measurements, and is an important parameter for designing the corresponding SKA survey.

2. Measurement of the size and ellipticity distributions of star-forming disk galaxies as a function of flux density and redshift. Lensing can only measure shapes of resolved galaxies; the size distribution is therefore a crucial ingredient in designing future surveys. We need to assess the degree of source overlap and any similar effects of confusion in the faint source population, and measure the "covering factor" of the FRI galaxies 
which will be masked out of the lensing analysis.

3. The refinement of shape-measurement techniques in Fourier space, which must proceed in parallel with the location of the sources in the image plane.

4. The effect of image processing (cleaning and calibration) on correlated noise in the radio images.

The imprint of gravitational lensing has been detected in the FIRST radio survey with a significance level of $3 \sigma$ (Chang et al. 2004). ASKAP should allow lensing measurements at roughly the same level as the current leading optical imaging surveys, covering hundreds of $\mathrm{deg}^{2}$ with perhaps one resolved source per $\operatorname{arcmin}^{2}$. Although ASKAP will not be competitive with the next generation of optical lensing surveys such as Dark Energy Survey and PanStarrs, ASKAP will pave the way for successful lensing experiments in the radio wavebands. 
4 Polarization Science

Lead authors: R. Taylor, B. Gaensler

Contributing authors: J. Brown, M. Haverkorn, R. Kothes, T. Landecker, J. Stil, M. Wolleben

\subsection{ASKAP All-Sky Polarization Survey}

ASKAP will be both a technical and scientific pathfinder to the Square Kilometre Array. One of the five key-science drivers for the SKA is to understand the origin and evolution of cosmic magnetism. The prime observational approaches to this key science will be a deep survey of polarized emission from compact extragalactic sources (Beck \& Gaensler 2004), and of the diffuse polarized radiation from the Galaxy. This data set will provide

- a deep census of the polarization properties of galaxies as a function of redshift (secured through complementary H I or optical surveys),

- a dense grid of Faraday rotation measures to over 500,000 background radio sources

- all-sky Faraday Rotation image of the 3 -dimensional structure of the diffuse magnetoionic medium of the Galaxy.

The Rotation Measure (RM) of a polarized radio signal is derived from the variation in wavelength of the polarization angle of linearly polarization, $\phi$, given by

$$
\phi=\phi_{\circ}+\lambda^{2} 0.812 \int n_{e} \mathbf{B} \cdot \mathbf{d l}=\phi_{\circ}+\lambda^{2} \mathrm{RM}
$$

where $n_{e}\left[\mathrm{~cm}^{-3}\right]$ is the electron density and $\mathbf{B}[\mu \mathrm{G}]$ is the magnetic field along the propagation path $\mathbf{d l}[\mathrm{pc}]$. A dense, all-sky grid of RM values to background radio sources and all-sky images of the RM of the polarized emission from the Galaxy, are thus powerful and unique probes of cosmic magnetism in the Milky Way Galaxy, the intergalactic medium, and in extragalactic radio sources.

\subsubsection{Compact Sources}

The SKA will probe to levels of 10s of nano-Jy, and measure the polarized intensity and Rotation Measure for $\sim 10^{7}$ sources with an average separation on the sky of about $1^{\prime}$.

Currently the deepest large area survey of polarized emission from compact extragalactic sources is the NVSS (Condon et al. 1998), which measures integrated polarized intensity for the entire northern sky for sources down to $\sim 1 \mathrm{mJy}$. Between now and 2010, the major step in this field will be the Galactic ALFA Continuum Transit Survey (GALFACTS), which will use the L-band Feed Array on the Arecibo telescope to carry out a spectro-polarimetric survey of the sky visible from Arecibo $\left(5.14 \mathrm{sr}^{-1}\right)$ over a bandwidth of $300 \mathrm{MHz}$ to a $1 \sigma$ sensitivity of $0.1 \mathrm{mJy}$. GALFACTS will take 1600 hours of observations and five years to complete (Taylor et al. 2006). Multiple spectral channels across the $300 \mathrm{MHz}$ band will allow RMs to be derived to a flux density limit of about $0.5 \mathrm{mJy}$, or about $5 \times 10^{4}$ sources. Deep polarimetric imaging with the DRAO 
telescope (Taylor et al. 2007) shows an excess population of polarized sources above the expectation based on the known properties of strong sources, that appears at flux densities less than about $30 \mathrm{mJy}$ (see Fig. 18), suggesting that polarized emission in the sub-mJy regime will be dominated by galaxies.

While GALFACTS will be a major step over our current knowledge (a few $10^{3}$ known RMs), there will still be a large observational gap between GALFACTS and the SKA. ASKAP must take the next step in scientific pathfinding to the SKA. Assuming an elevation angle limit of $15^{\circ}$, the declination range visible to ASKAP is $+48^{\circ}$ to $-90^{\circ}$, which covers a solid angle of $3.8 \pi$ or $10.95 \mathrm{sr}$, a very large fraction, $87 \%$, of the total sky (compared to $31 \%$ for Arecibo; see Fig. 16).

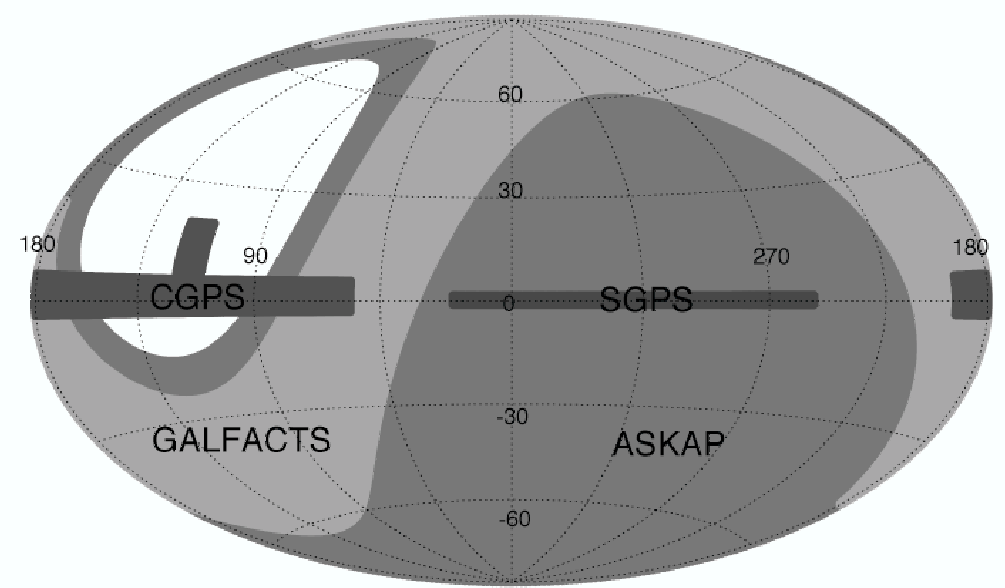

Fig. 16 Sky coverage of ASKAP (medium grey) in comparison with recent large-array polarization surveys. The Canadian Galactic Plane Survey (CGPS; $55^{0}<l<195^{0},-3.5^{\circ}<b<$ $5.5^{\circ}$, plus a latitude extension $110^{\circ}<l<117^{\circ}, 5^{\circ}<b<1^{\circ}$; dark grey) is nearing completion with observations from the Dominion Radio Astrophysical Observatory. Observations for the Southern Galactic Plane Survey (SGPS; $253^{\circ}<l<20^{\circ},|b|<1.5^{\circ}$; dark grey) were completed in 2002 using the Australia Telescope Compact Array. The Galactic Arecibo L-band Feed Array Continuum Transit Survey (GALFACTS; $0^{\circ}<\delta<38^{\circ}$; light grey) will be commencing observations in 2008. All surveys have or will have multiple frequency bands and full Stokes coverage.

The key experiment for studying magnetic fields with ASKAP will be to image the entire southern sky at $1.4 \mathrm{GHz}$ to a $1 \sigma$ sensitivity of $50 \mu \mathrm{Jy}$ in full Stokes continuum emission. For ASKAP, this would be achieved in a one-hour integration per pointing, which for a field of view of $30 \mathrm{deg}^{2}$ implies a total survey time of 5-6 weeks. ${ }^{4}$ This survey will be a factor of two deeper than GALFACTS, providing a factor of $\sim 5$ more sources per unit solid angle: a dense grid of background RM measurements, probing to flux densities that begin to significantly sample the polarized emission from star-forming galaxies.

4 Obviously much deeper surveys could be reached in longer integrations, but the resulting Stokes $I$ images would be heavily confused. Also, an initial RM grid from ASKAP would provide such a large leap forward in our understanding of the polarized sky, that we would need to properly digest these new data before knowing how best to survey deeper. 


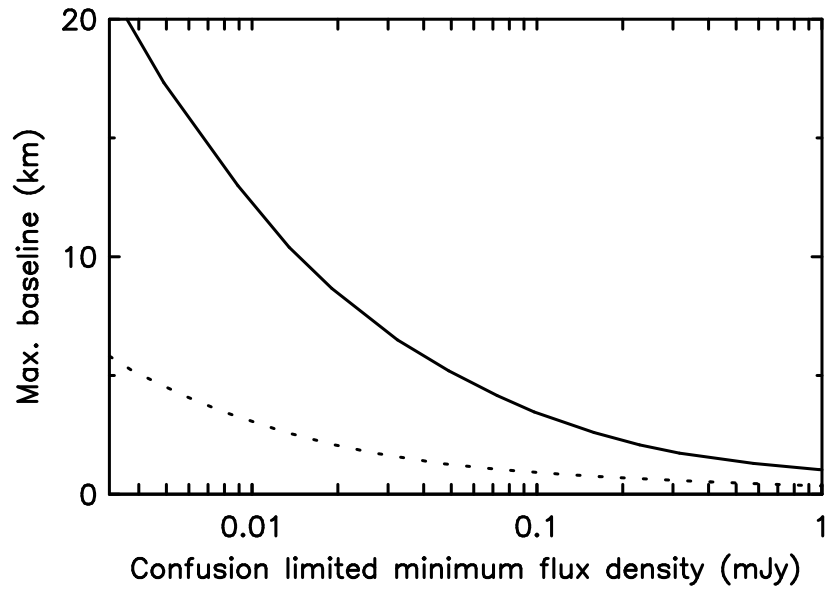

Fig. 17 Maximum baseline required to achieve images that are not limited by source confusion at $1.4 \mathrm{GHz}$ as a function of continuum sensitivity. For $1 \sigma$ noise of $50 \mu \mathrm{Jy}$, a maximum baseline of at least $6 \mathrm{~km}$ is required.

Fig. 17 shows the maximum baseline required to produce images that are not confusion limited as a function of sensitivity. An all-sky survey with $50 \mu \mathrm{Jy}$ rms requires at least a $6 \mathrm{~km}$ baseline. More sensitive observations of smaller regions will become confusion-limited quickly. A minimum baseline of at least $10 \mathrm{~km}$ (corresponding to angular resolution of better than $5^{\prime \prime}$ at $1.4 \mathrm{GHz}$ ), would allow noise-limited imaging down to about $10 \mu \mathrm{Jy}$.

\subsubsection{Diffuse Polarized Emission}

Existing studies of the diffuse emission from the Milky Way are limited in angular resolution or in sky coverage. Only one full-sky survey exists, with an angular resolution of $30^{\prime}$, and at a single frequency $(1.4 \mathrm{GHz})$. An extensive area of the northern sky has been covered by the Effelsberg Medium Latitude Survey, also at $1.4 \mathrm{GHz}$. Highresolution studies are confined to the Galactic plane, and all suffer to some degree from absence of information on the longest baselines. The first survey of the Galactic plane to have full representation of broad structure is the forthcoming survey from the CGPS.

An ASKAP all-sky survey should have full baseline coverage to fully sample the structure of the diffuse Galactic polarized emission. To achieve full baseline coverage ASKAP will need some baseline overlap with the available single antennas. Assuming that data can also be collected with the Parkes 64-m telescope, ASKAP will need some baselines as short as $32 \mathrm{~m}$.

Experience with aperture synthesis surveys shows very clearly that interpretation is usually impossible, and always difficult, with interferometer data only. We will need absolutely calibrated single-antenna observations that can be combined with ASKAP data. To provide a data set to underpin interferometric surveys with ASKAP and the new generation of high-resolution survey telescopes (EVLA, SKA), we should make systematic single-antenna spectro-polarimetric surveys in the northern and southern hemispheres over the entire range $300 \mathrm{MHz}$ to $3 \mathrm{GHz}$. 
4.2 Polarization of Extragalactic Radio Sources

Counting radio sources as a function of flux density is a powerful method to study the luminosity evolution of radio galaxies, even in the absence of redshift information. Analysis of these source counts suggests a shift in the dominant population of radio sources around $1 \mathrm{mJy}$, where brighter radio sources are predominantly accretion-powered and driven by active galactic nuclei, and fainter radio sources are predominantly powered by star formation. A significant population of radio-quiet AGN may also be present at faint flux levels. The number of faint polarized radio sources is not only important as the basis of the rotation measure grid; it also provides valuable information about the cosmic evolution of large-scale magnetic fields in galaxies.

The fractional polarization, $\Pi$, of extragalactic sources is typically only a few percent, so the signal-to-noise ratio in polarized flux is always a factor $\sim 50$ below the signal-to-noise ratio in total intensity. There are particular problems in measuring polarized flux if the signal-to-noise ratio is low, because the mean of the noise in $P=\sqrt{\left(Q^{2}+U^{2}\right)}$ is non-zero (polarization bias), and the statistical distribution of the noise depends on the signal-to-noise ratio. Signal-to-noise ratio and completeness requirements have limited investigations of the polarization properties of extragalactic sources to the bright $\left(S_{1.4 \mathrm{GHz}} \gtrsim 80 \mathrm{mJy}\right)$ radio source population, dominated by powerful, radio loud AGN. There are some indications from these bright sources that at least fainter steep-spectrum radio sources are more highly polarized (Mesa et al. 2002; Tucci et al. 2004), up to a median $\Pi \sim 1.8 \%$ in the flux density range $100-200$ mJy.

Figure 18 shows a flux-limited sample of polarized sources in the DRAO ELAIS N1 deep field with central noise level $74 \mu \mathrm{Jy}$ (Taylor et al. 2007). The fractional polarization of this deep sample was analyzed through a Monte Carlo analysis by creating synthesized source lists with the same noise properties as the data, including variation of the noise across the field. Each synthesized source list was created assuming a particular distribution of $\Pi$ for the polarized sources. The Monte Carlo simulations incorporate all known effects of the noise and the detection limit. The distribution of the data in the plane of $\log$ (total flux density) - $\log$ (polarized flux density) can be compared directly to the simulations. Figure 18 shows the distribution that assumes the flux-independent $\Pi$ distribution derived by Beck \& Gaensler (2004). Clearly, this distribution does not represent the faint sources very well, although it is consistent with sources brighter than $\sim 80 \mathrm{mJy}$, for which it was derived. Taylor et al. (2007) performed a maximum likelihood fit of a large set of synthesized source lists to the data assuming a set of $\Pi$ distributions based on a Gauss-Hermite series with lowest order symmetric deviations from a Gaussian (van der Marel \& Franx 1993). The best fitting distribution is nearly Gaussian of standard deviation $\sigma_{\Pi}=7.5 \% \pm 1.5 \%$ with the parameter for deviation from a Gaussian, $h_{4}$, not significantly different from 0 . For a Gausian $\Pi$ distribution, the median is $\frac{2}{3} \sigma_{\Pi}$. A two-dimensional Kolmogorov-Smirnov test (Peacock 1983) suggested that a flux-dependent $\Pi$ distribution is required by the data.

Figure 19 shows first results of a similar analysis of NVSS sources (Stil et al. 2008). Here, the maximum likelihood fit was done as a function of flux density. Although the NVSS has much higher noise than the DRAO ELAIS N1 deep field, here too a increase in the width of the $\Pi$ distribution is found, similar to the result in Figure 18. Note that the slight increase in median fractional polarization for sources brighter than $100 \mathrm{mJy}$ found by Mesa et al. (2002) and Tucci et al. (2004) is also reproduced. This kind of 


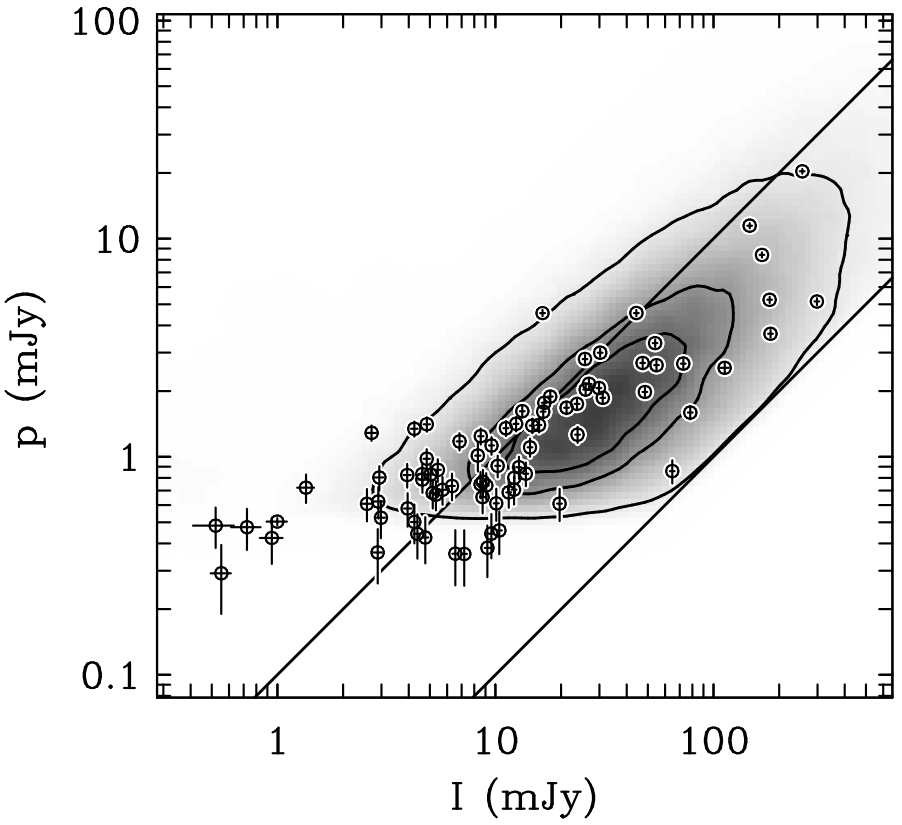

Fig. 18 Distribution in $\log (I)-\log (P)$ of observed sources (open circles), compared with a simulated probability distribution (grayscale) that assumes the $\Pi$ distribution for strong sources from Beck \& Gaensler (2004). The inner contour contains $25 \%$ of the simulated sources, the middle contains $50 \%$ and the outer contour encompasses $90 \%$. The two lines mark the loci of sources with $\Pi=0.01$ and $\Pi=0.1$. Figure from Taylor et al. (2007).

analysis requires large samples of polarized sources observed with a high signal-to-noise ratio in polarized flux.

The increased fractional polarization of faint sources implies significantly larger numbers of faint polarized sources than previously thought. Figure 20 shows polarized source counts extrapolated to lower flux density by convolving the $\Pi$ distribution with the total-intensity source counts in the same way as done by Beck \& Gaensler (2004). The newly-predicted Euclidean-normalized source counts are nearly flat in the range 2-100 mJy, which appears more consistent with polarized source counts derived from the NVSS by Tucci et al. (2004). We predict approximately $50 \%$ more sources with a polarized flux down to 1 mJy than Beck \& Gaensler (2004).

ASKAP will revolutionize this field by providing larger samples of polarized sources, to much fainter flux densities than currently possible. At a sensitivity level of $30 \mu \mathrm{Jy}$, ASKAP will begin to reveal the polarization of radio sources as faint as a few mJy, where star-forming galaxies begin to contribute measurably to the total radio source counts (Windhorst 2003). Models of the integrated polarized emission from disk galaxies, based on magnetic field strengths and geometries (Figure 21), in conjunction with analysis of the integrated polarization of nearby disk galaxies, will provide a theoretical basis for analysis of the magnetic properties of the large sample of distant star-forming galaxies that will be observed by ASKAP. The idealized model sketched in Figure 21 suggests that the $\Pi$ distribution of a large sample of randomly oriented disks has a minimum at $\Pi=0$. The presence of such a minimum in the distribution depends on the importance of Faraday depolarization and the importance of poloidal magnetic 

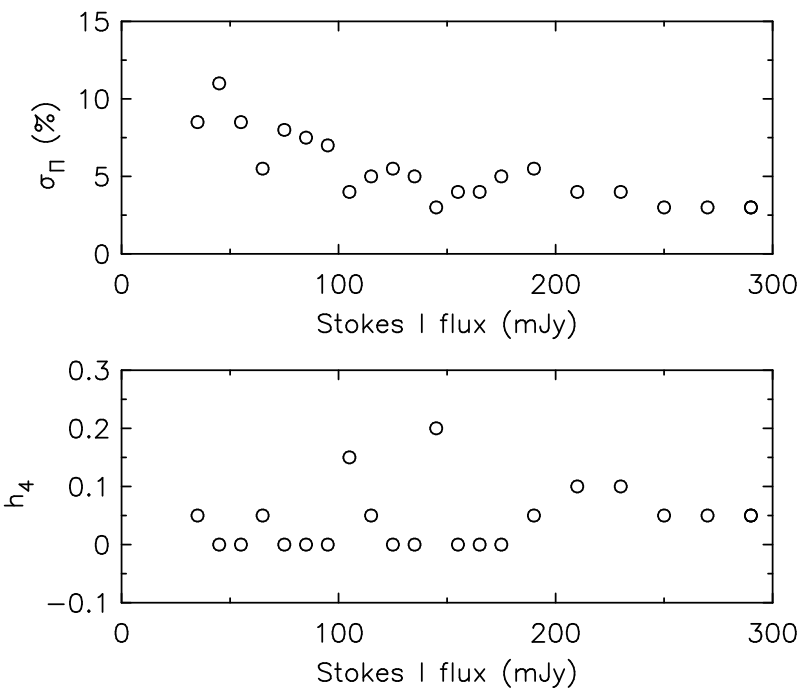

Fig. 19 Variation with flux density of the parameters that describe the best-fitting $\Pi$ distribution from maximum likelihood fits to NVSS sources. The parameters are a Gaussian standard deviation $\sigma_{\Pi}$, and the coefficient $h_{4}$, which quantifies lowest-order symmetric deviation from a Gaussian (Van der Marel \& Franx 1993). The median fractional polarization is $\frac{2}{3} \sigma_{\Pi}$ for a Gaussian distribution. The Gaussian width $\sigma_{\Pi}$ increases with decreasing flux density (Stil et al. 2008).

fields in the halo of these galaxies. We can measure this minimum by including the lowest-order antisymmetric deviations from a Gaussian $\left(h_{3}\right)$ in our Monte-Carlo analysis of a sufficiently large number of galaxies. The projected large frequency coverage of ASKAP will allow us to distinguish between highly frequency-dependent Faraday depolarization, and magnetic field structure that should not change with observing frequency. As we consider integrated quantities, our analysis is not affected by variation of resolution with frequency.

For the first time, ASKAP will provide polarization measurements for a large sample of disk galaxies, allowing us to investigate the large-scale magnetic field topology and Faraday depolarization for distant star-forming galaxies. We will also be able to study the polarization properties of AGN for different subsamples, e.g. depending on spectral index or rotation measure. This will extend the success of radio source counts in studying the luminosity evolution of radio galaxies to the evolution of magnetic fields with cosmic time.

\subsection{Galactic Polarization Science}

The study of Faraday rotation of background compact sources through the Galaxy, and of the polarized emission from the diffuse ISM and Galactic objects such as SNRs, pulsar wind nebulae and planetary nebulae, promises a wealth of advance in our knowledge of nature of the Galactic magnetic field and the role of magnetism in the evolution of the interstellar medium. Questions in Galactic astrophysics that an ASKAP polarization survey may address include: 


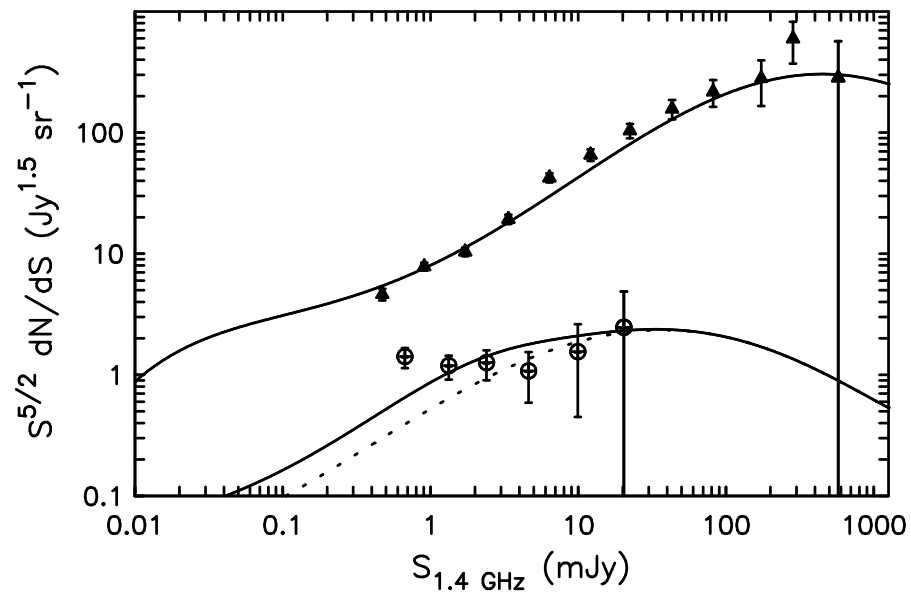

Fig. 20 Stokes $I$ and polarized differential source counts. The top solid line is the Stokes I curve from Windhorst et al. (1990). The predicted polarized counts (lower solid curve) is derived by convolving the total intensity source counts with a $\Pi$ distribution that becomes broader for fainter sources, consistent with the results shown in Figures 18 and 19. Polarized source counts assuming the flux-independent $\Pi$ distribution of Beck \& Gaensler (2004) are also shown (dotted curve). The data points are the observed Stokes $I$ and polarized source counts from the DRAO ELAIS N1 Deep Field (Taylor et al. 2007).

1. What is the vertical distribution of the Magneto-Ionic Medium? Is the scale height longitude dependent?

2. Is there a detectable transition between disk and halo? Either a transition in field structure or a transition in turbulent structure?

3. Can we correlate (or anti-correlate) polarization features with other ISM tracers such as atomic, molecular gas, dust, $\mathrm{H} \alpha$ ? Such correlations are very useful in establishing the distance to polarization features.

4. Can we use polarization observations to detect the million-degree gas? If so, can we determine the filling factor of the million-degree gas?

5. There are definitely polarization structures which are not correlated with anything else. Some have scales of tens of degrees. What are they?

6. What probes can we find to help us measure the magnetic field in different places? Supernova remnants? Planetary Nebulae? H il regions?

7. Are some polarization structures purely (or even largely) produced by variation of magnetic field, as opposed to electron density? What are they?

8. Can Faraday tomography reveal the characteristics and structure of the magnetoionic medium?

9. Can we relate the small-scale structure seen by probing the RM through the entire Galaxy (seen with extragalactic sources) with features seen in the extended emission?

10. What are the filling factors of the Warm Ionized Medium and the magnetic field?

11. What is the variation of magnetic field strength with galactocentric radius?

The results from recent and on-going polarimetric surveys point the way to ASKAP Galactic polarization science and the observational requirements. 


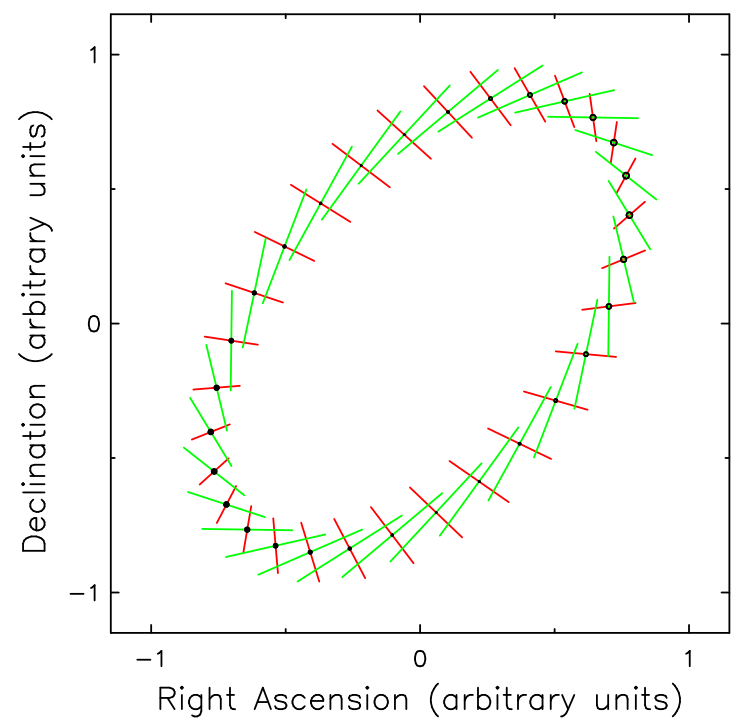

Fig. 21 Schematic representation of the polarized intensity along a circle at some radius in the disk of a spiral galaxy. Green: projected magnetic field vectors. Red: vectors of polarized intensity from synchrotron emission. The black dots indicate the magnitude of the component of the magnetic field along the line of sight. The integrated emission over the face of a disk results in zero net polarized flux density. However, for an inclined disk the integrated polarized flux density is non-zero with a net alignment of the polarization vectors along the apparent minor axis of the disk (Stil et al. 2008).

\subsubsection{The Galactic Magnetic Field}

The Galactic magnetic field is a fundamental component of the interstellar medium, and plays a critical role in the formation and evolution of structures in the Galaxy. The field is often considered composed of two components: a large-scale field with scale sizes on the order of kiloparsecs, and a small-scale or turbulent field with scale sizes on the order of hundreds of parsecs and smaller. The field is primarily concentrated in the disk with the large-scale field having both radial and azimuthal components (Beck et al. 1996). The small-scale field is usually assumed to be isotropically distributed (Heiles 1996), but there has been some evidence to suggest it is fundamentally tied to the large-scale field (Brown \& Taylor 2001). The field extends into the halo with a magnitude roughly one tenth of that in the disk (Han \& Qiao 1994). However, detailed studies of either the halo field or the transition from the disk to the halo have yet to be carried out. Understanding the topology of the field in the disk and the halo is essential to understanding the evolution of the field (Zweibel \& Heiles 1997) and the Galaxy itself (Shukurov 2005).

The field is most often studied via observations of rotation measure (RM), the measurable consequence of Faraday rotation (see equation 5). The RM integral is over the path from the source to the observer. Two sources of polarised radiation frequently used to probe the field in this way are pulsars and external galaxies (EGS). Ideally, reconstruction of the Galactic magnetic field should utilize information from both of these sources. However, until recently there have been very few EGS RMs available at low latitude that might be used to study the field in the disk in conjunction with the 


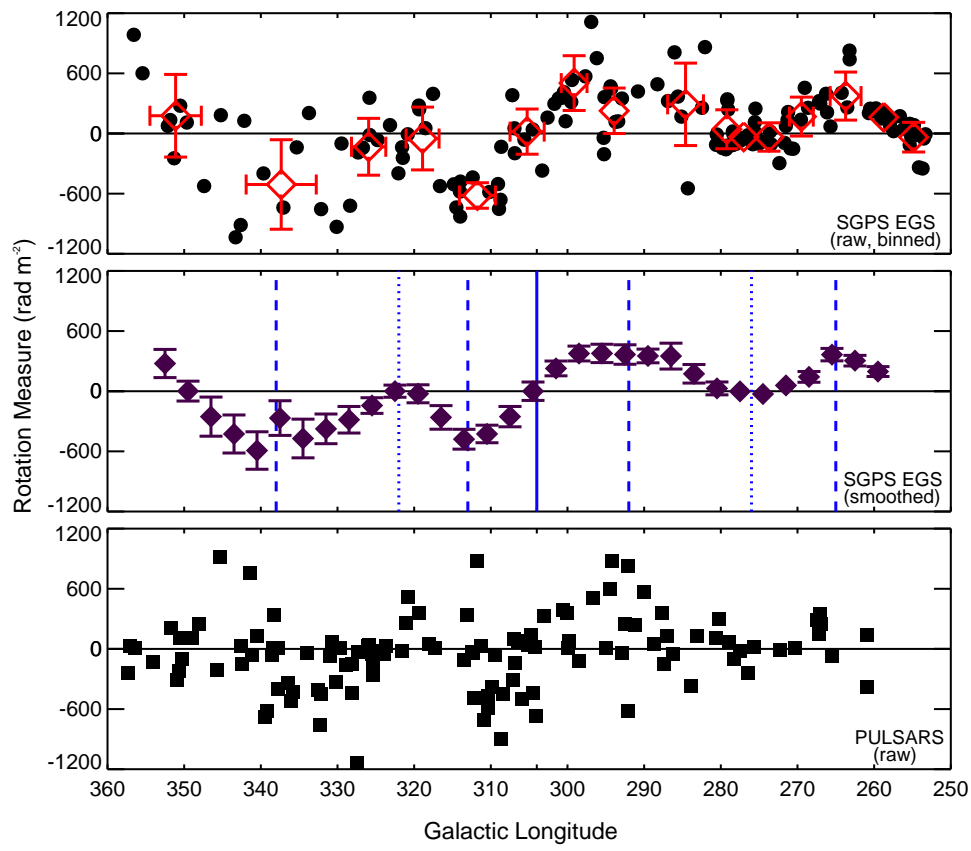

Fig. 22 RM versus Galactic longitude for EGS and pulsar RMs in the Southern Galactic Plane Survey (SGPS), from Brown et al. (2007). Top panel: circles represent the individual SGPS EGS RMs (errors are smaller than the symbol size), while open diamonds represent data averaged into independent longitude bins containing nine sources each. Where symbol size permits, the error bars are the standard deviation in longitude and RM for each bin. Middle panel: diamonds represent boxcar-averaged SGPS EGS data over 9 degrees in longitude with a step size of 3 degrees. In contrast to the binned data in the top panel, the error bars are the standard error of the mean. The solid line marks the approximate longitude of transition from predominantly positive $\mathrm{RMs}$ to negative $\mathrm{RMs}\left(\ell \sim 304^{\circ}\right)$. Dotted (dashed) lines mark approximate longitudes of minimum (maximum) $|\mathrm{RM}|$ in SGPS data. Bottom panel: squares represent individual pulsars with known RMs in the SGPS region; errors are smaller than the symbol sizes.

pulsars. Studies that have utilized pulsar RMs alone are constrained by the comparatively sparse sampling of pulsars on the sky, which can make it difficult to map the field in complicated regions.

Recent surveys of the Galactic plane at high resolution and at multiple wavelengths have assisted greatly in the study of the Galactic magnetic field by addressing the previous paucity of low-latitude EGS RM data (e.g. Brown \& Taylor 2001). The first large-scale observing survey to image the Galactic plane is the Canadian Galactic Plane Survey (CGPS; Taylor et al. 2003), which obtained roughly 1 RM source per square degree. Complementing the CGPS in the southern hemisphere is the Southern Galactic Plane Survey (SGPS; McClure-Griffiths et al. 2005; Haverkorn et al. 2006), which yielded 1 RM source every 2 square degrees. These new data-sets such as shown in Figure 22 strikingly confirm the presence of a large-scale coherent field. However, the statistics of such RM data are insufficient to derive unique solutions for the field geometry, and usually rely on pre-imposed assumptions regarding the geometry of the spiral arms and the thickness of the disk. Furthermore, the latitude coverage of these 
surveys is substantially incomplete, with currently very limited RM coverage in the first and third quadrants. Finally, these data lack the sample sizes needed to map complicated small-scale features, and to separate the magnetic field in arm vs. interarm regions.

The order-of-magnitude improvement in the size of RM samples offered by ASKAP will allow us to overcome these current limitations in our understanding. Based on the fractional polarization distribution of faint radio sources (Taylor et al. 2007), with a $1 \sigma$ sensitivity of $50 \mu \mathrm{Jy}$, a survey with ASKAP will yield $70 \mathrm{RMs}$ per square degree, for a typical separation on the sky between RM measurements of less than $10^{\prime}$.

\subsubsection{Disk-Halo Transition and the Galactic Magnetic Field at High Latitudes}

There have been several studies targeting the large-scale field in the disk (e.g. Rand \& Lyne 1994; Indrani \& Deshpande 1998; Brown \& Taylor 2001; Brown et al. 2007). However, there have been very limited studies of the halo field (Han \& Qiao 1994), and even less study of the disk-halo interface (Beck 2001). As a corollary, even basic properties such as the scale-height of the magneto-ionic medium (MIM) are only vaguely understood.

The transition between the disk and the halo is an active region containing unique physics. Hydrostatic equilibrium modeling predicts a more or less quiescent magnetic field in the halo, transforming to a turbulent field in the disk at a transition height of about 500 - 1000 pc (Kalberla \& Kerp 1998). A change in the character of the magnetic field is indeed seen in external galaxies (Dumke et al. 1995) and also in the Milky Way there is evidence that the polarization characteristics of the diffuse polarized intensity change at $|b| \approx 30^{\circ}$ (Wolleben et al. 2006; see Figure 23). At intermediate latitudes, 'Galactic chimneys' are thought to transfer ionising photons from the OB stars in the disk to the halo, providing a major part of the ionization of the halo (Reynolds 1985). Numerical simulations indeed show that supernova explosions in the disk cause large-scale gas streams into the halo, where ram pressure dominates over magnetic pressure and the magnetic field is swept into magnetic bubbles (de Avillez et al. 2004). The imprints in the Galactic magnetic field left by these processes give us unique knowledge about the magnetism and gas dynamics in a bubble or chimney. External galaxies often show magnetic field structures inclined or even perpendicular to the plane (Beck 2001). However, in only one Galactic superbubble (in the Northern sky) has the magnetic field been studied (West et al. 2007).

To date, the only data readily available for probing the Rotation Measure variation over this interface are from measurements of background sources in the CGPS. Figure 24 shows averaged-smoothed data for the latitude extension of the CGPS. From this graph, the "half-height" latitude is estimated at $4.5^{\circ}$. If we assume a Galactic radius of $20 \mathrm{kpc}$, and that the disk is symmetric, then the scale height corresponding to $b=4.5^{\circ}$ is $1.2 \mathrm{kpc}$, consistent with the calculations of Han \& Qiao (1994). However, this does not take into consideration the warp of the disk. While data from GALFACTS will allow additional probing in a limited longitude range both above and below the disk in the first quadrant and at the anti-center, ASKAP allows for the possibility of continuous probing in longitude for 3 of the 4 quadrants. With such longitude coverage, it should be possible to fully map the magneto-ionic disk in these quadrants, and thus reveal the signature of the warp, should it exist in the MIM, as well as provide insight into how the transition between the disk and halo occurs. 


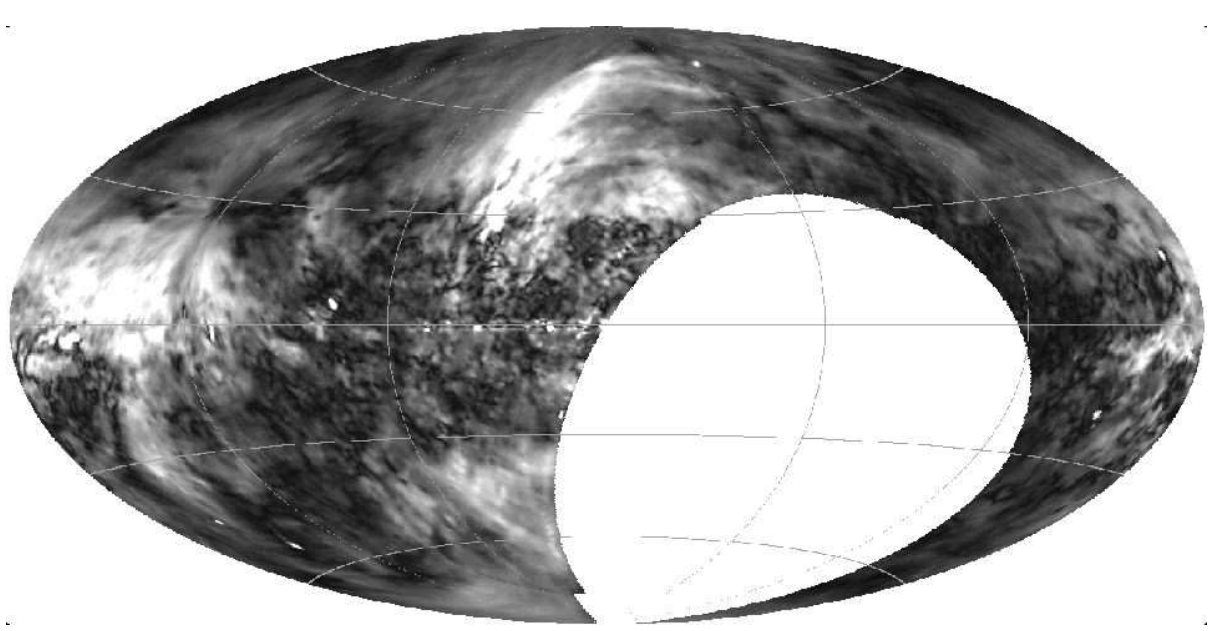

Fig. 23 All sky diffuse polarization at $1.4 \mathrm{GHz}$ from the survey of Wolleben et al. (2006). The image is in Galactic coordinates with $l=0^{\circ}, b=0^{\circ}$ at the centre.

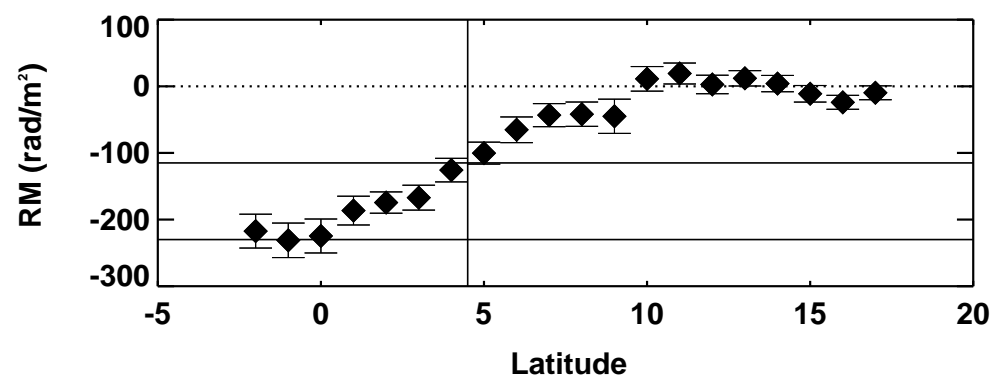

Fig. 24 Variation of mean RM versus Galactic Latitude at $\ell=120^{\circ}$ from the CGPS. (J. Brown, University of Calgary).

Even less attention has been given to the Galactic magnetic field at high latitudes (see Han \& Qiao 1994), because of the sparse sampling of RMs over most of the rest of the sky ( $1 \mathrm{RM}$ per $\sim 40 \mathrm{deg}^{2}$ ). As a corollary, the scale height of the magneto-ionic medium is only vaguely understood.

There are two important motivations for trying to obtain a better understanding of the high-latitude field. First, because this part of the sky is free from turbulent and tangled fields as are seen in the plane, overall patterns in RM are easy to identify (e.g. Frick et al. 2001). Second, unique insights on Galactic magnetism, and on the mechanisms which maintain it, can be provided by studying the parity of the field across the Galactic plane. Specific issues which can be addressed by such studies are as follows.

- The symmetry of the vertical $\left(B_{z}\right)$, azimuthal $\left(B_{\phi}\right)$ and radial $\left(B_{r}\right)$ components of the field above vs. below the plane discriminates between overall field geometries, and hence between various primordial field and dynamo models. For example, a reversal in $B_{\phi}$, but not in $B_{z}$, between positive and negative Galactic latitudes would indicate either a primordial vertical field, or an A0 mode (antisymmetric field with 


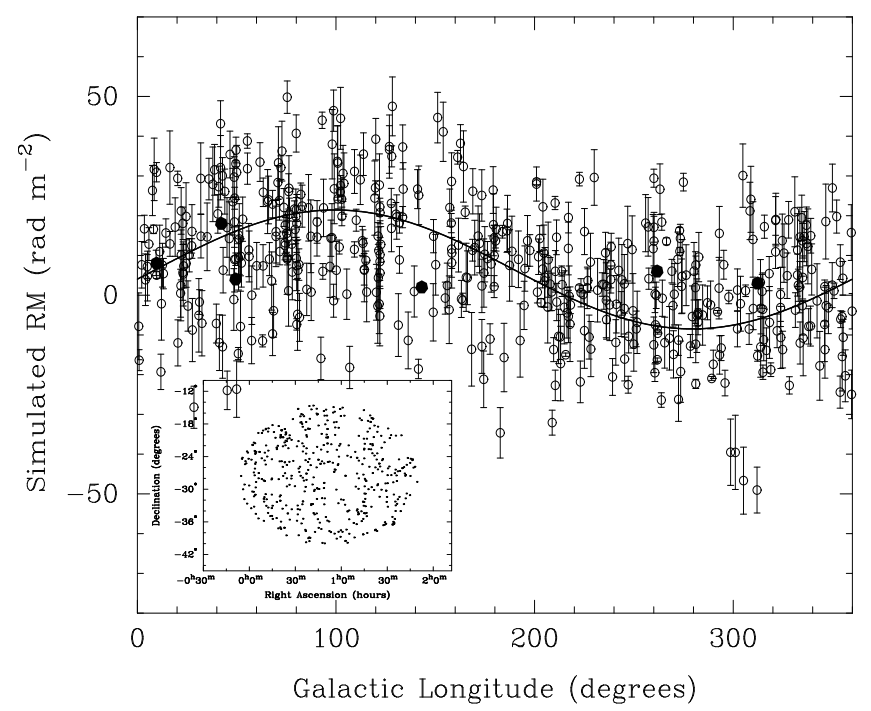

Fig. 25 The distribution in $\ell$ of 500 highly polarized NVSS sources covering the South Galactic Cap, the RM of each of which has recently been measured with the ATCA (Mao et al., in preparation). For each source, a simulated RM value is plotted, assuming a simple threedimensional magnetic field geometry for the disk and halo; the filled circles show the six previously known RMs in this region of the sky. In the simulation, the field parallel to the disk reveals itself as a sinusoidal dependence of RM vs. $\ell$, while the constant offset in RM is the signature of a non-zero value for $B_{z}$. The phase and amplitude of the sinusoid then yield the pitch angle and strength, respectively, of the horizontal field above the disk. Inset: The distribution of the 500 NVSS sources on the sky.

a dipolar structure) as would result from weak differential rotation (Ferrière 2005). Alternatively, a reversal in $B_{z}$ across the plane, but not in $B_{\phi}$ or $B_{r}$, corresponds to a quadrupolar field, as results from strong shear (Zweibel \& Heiles 1997).

- The strength of $B_{z}$ is a diagnostic of the origin of the magnetic field. For a Galactic disk of diameter $2 R$ and thickness $2 h$, kinematic dynamo models predict $B_{z} / B_{r} \approx$ $\sqrt{h / R} \approx 0.3$. However, if $B_{z} \ll B_{r}$, this can indicate that there is a significant primordial component to the magnetic field (Ruzmaikin et al. 1998).

- The strength of $B_{z}$ compared to the horizontal component regulates the efficiency with which cosmic rays flow from the disk into the halo and then into the intergalactic medium (e.g. Carilli et al. 1992). Conversely, vertical magnetic fields of fluctuating sign may result from Parker instabilities or galactic winds. In a few edge-on galaxies, polarization data suggest strong vertical fields (e.g. Tüllmann et al. 2000), but the situation for our Galaxy is unclear.

As explained in Section 4.1.1, ASKAP can provide the densely sampled RM grids at high latitude needed to address these issues. Figure 25 illustrates a forthcoming analysis of 500 RMs measured with the ATCA covering the South Galactic Cap. The 200 hours of observing time that underlie this data-set should provide some important first constraints on the vertical field geometry of our Galaxy. However, in just 80 hours, ASKAP will measure $\sim 10^{4} \mathrm{RMs}$ in this same region, clearly providing a colossal improvement in the effective sensitivity and angular resolution of a smoothed Galactic RM map at these latitudes. 


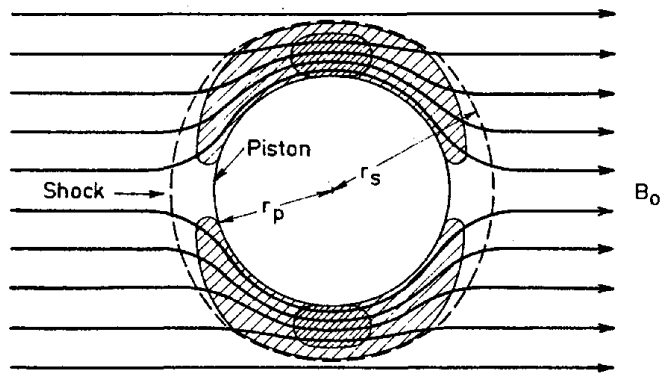

(a)

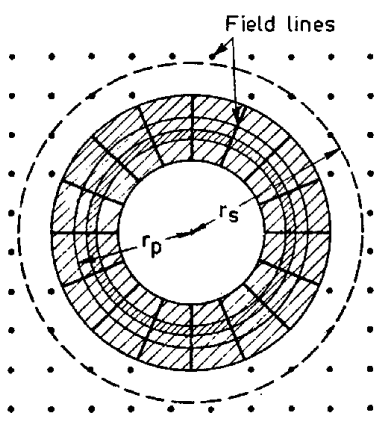

(b)

Fig. 26 Model of a shell-type SNR expanding in a homogeneous ambient magnetic field, viewed perpendicular to (left) and along (right) the magnetic field lines (Whiteoak and Gardner 1968; reproduced by the kind permission of J.B. Whiteoak).

Radio polarization observations of shell-type SNRs can also be a powerful tool to probe the magnetic field into which these objects are expanding. Shell-type SNRs can be used as magnifying glasses of that magnetic field, since it is frozen and compressed into the expanding shell of the SNR (see Fig. 26). Radio polarization observations of SNRs can be used to determine the 2 and 3 dimensional magnetic field structure of its environment. We can observe the SNR's structure projected to the plane of the sky in total power and polarization, the latter after correcting for Faraday rotation. From this we can extrapolate the ambient magnetic field projected to the plane of the sky. The third dimension can be determined by studying the structure of rotation measure over the SNR. This is nicely demonstrated in a study of the Galactic SNR CTB104a (Uyaniker, Kothes, \& Brunt 2002). The internal magnetic field structure of the SNR was derived from a rotation measure gradient over the SNR (see Fig. 27). The internal structure implied a magnetic field anomaly in the area around the remnant which was also evident in a study of rotation measures of polarized background sources (Brown \& Taylor 2001).

Both of these studies require knowledge about the polarized emission on small and large scales, since SNRs can be quite large in angular extent if they are nearby or very small if they are far away on the other side of the Milky Way.

\subsubsection{Diffuse Polarized Emission in the Milky Way}

\subsubsection{The Magneto-Ionic Medium}

Experience over the past 15 years in study of the polarized emission of the Galaxy has shown that the appearance of the polarized sky is dominated by Faraday rotation, except at the shortest wavelengths. The major scientific focus is study of the Faraday rotating medium, and only secondarily the study of the synchrotron-emitting regions themselves, which can in general be regarded as smooth. 


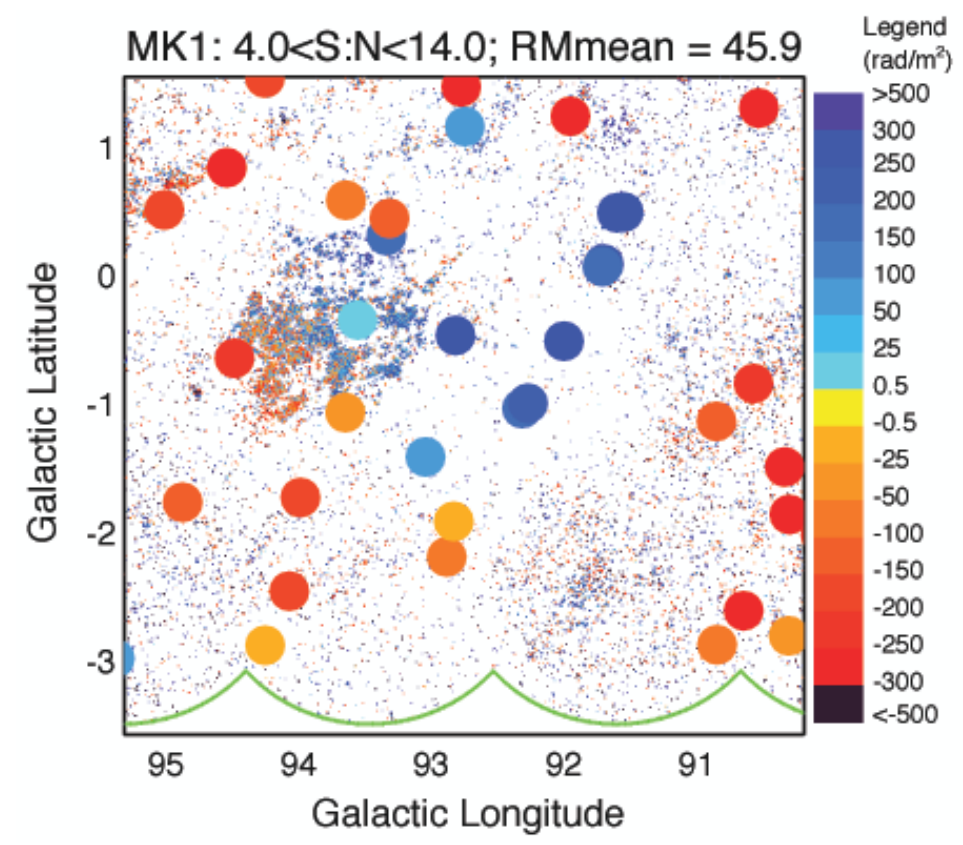

Fig. 27 Map of rotation measure in the area of the supernova remnant CTB104a taken from the Canadian Galactic Plane Survey with overlaid point source rotation measures. The RM of background sources is predominantly negative in this part of the Galaxy. The cluster of positive values, possibly associated with the SNR, indicates a magnetic anomaly in this area of our Galaxy. (J. Brown, University of Calgary)

Observations of external galaxies show that their synchrotron emission is quite smooth, and it has been assumed that this applies to our Galaxy as well. This was dramatically confirmed in 2006 with the publication of the WMAP 3-year results. The 23 $\mathrm{GHz}$ image shows, at an angular resolution of about 2 degrees, that the Galactic emission is smooth. Despite the smooth nature of the synchrotron emission, observations at lower frequencies, from $300 \mathrm{MHz}$ to $2.7 \mathrm{GHz}$, made with various telescopes, both single antenna (Parkes, Effelsberg) and synthesis telescopes (DRAO, ATCA, WSRT) show extensive small-scale chaotic structure (unrelated to features seen in total intensity) that are produced by Faraday rotation.

The study of diffuse polarized emission in this frequency range requires a careful balance of sensitivity and angular resolution. There exists a "polarization horizon", a distance beyond which polarized features can no longer be detected because of a combination of depth depolarization and beam depolarization, with beam effects dominating. Because the polarization horizon is partly determined by depth depolarization, it is somewhat direction dependent. Fig. 28 shows the polarization horizon effect in terms of the limit on the depth of current studies. Identified regions of Faraday rotation structure in the diffuse emission lie within about $2 \mathrm{kpc}$ of the Sun. Using the DRAO Synthesis Telescope at $1.4 \mathrm{GHz}$ with its $1^{\prime}$ beam, the distance to the polarization horizon is about $2 \mathrm{kpc}$ towards longitude $80^{\circ}$. This distance gradually increases towards the anticentre because the direction of the regular component of the field gradually becomes perpendicular to the line of sight. Towards the inner Galaxy we have studied 


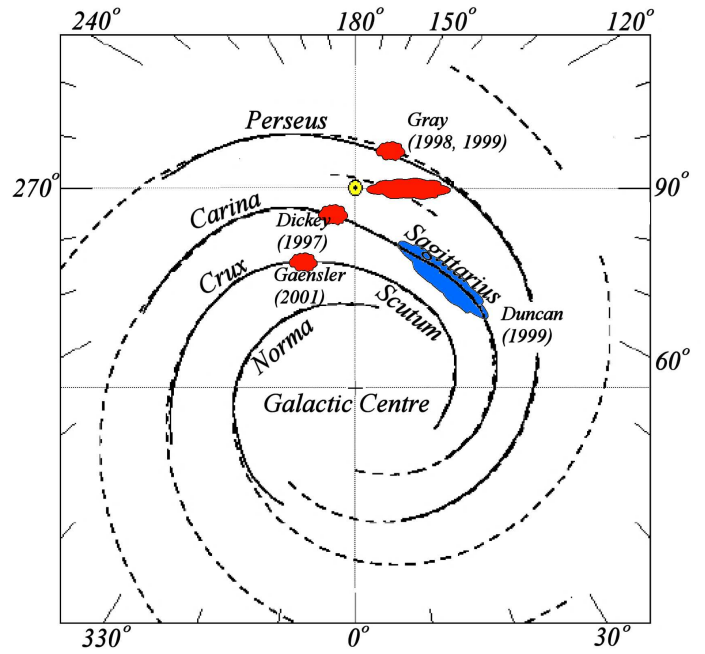

Fig. 28 The effect of the "Polarization Horizon" is to reduce the depth to which Faraday rotation structures are visible in the plane of the Galaxy. High angular resolution and observing frequencies up to $3 \mathrm{GHz}$ are required to probe deep in to the disk of the Galaxy.

only a very small fraction of the Galactic disk because of polarization horizon effects. A maximum baseline of at least $3 \mathrm{~km}$ is required to "see" into the Galaxy.

\subsubsection{Rotation Measure Synthesis}

In recent years new spectrometer backends have been developed that measure polarization over a broad band and a large number of channels. This makes rotation measure synthesis (RM-synthesis, in short) a potentially powerful tool for the data analysis. The idea behind RM-synthesis is that one de-rotates, for every pixel in each channel, the polarization vector in order to correct for Faraday rotation along the line-of-sight. This is done by calculating, for each pixel, the integrated complex polarization $(P=Q+i U)$ assuming a spectrum of $\mathrm{RM}$ values. By plotting $|P|$ versus the assumed $\mathrm{RM}$ one obtains an RM-cube.

RM-Synthesis can thus be a powerful means to unravel polarized emission layers along a given line-of-sight, thereby providing a three-dimensional deconvolution of the synchrotron emissivity, magnetic fields and ionized hydrogen in the Galaxy. RMSynthesis can be performed on polarization data cubes (two spatial coordinates versus frequency) on a pixel-by-pixel basis (Brentjen \& de Bruyn 2005). A reasonably wide bandwidth with sufficient number of frequency channels is required. In the case of synthesis observations, which suffer from incomplete coverage of spatial frequencies (or missing large scale structures), polarization angles are arbitrary. It will therefore be desirable to include information from single-dish surveys to complement polarization cubes from ASKAP.

The following estimates of instrumental requirements are based on approximations given in Brentjen \& de Bruyn (2005). The key instrumental parameters in planning an RM-synthesis experiment are the squares of the channel width $\delta \lambda^{2}$, the bandwidth 
$\Delta \lambda^{2}$, and the shortest wavelength $\lambda_{\min }^{2}$. The RM resolution of the data $\delta \phi$ depends on the bandwidth utilized:

$$
\delta \phi \approx \frac{2 \sqrt{3}}{\Delta \lambda^{2}}
$$

The shortest wavelength determines the largest scale in RM-space to which one is sensitive,

$$
\max \text {-scale } \approx \frac{\pi}{\lambda_{\min }^{2}},
$$

and the maximum observable Faraday depth depends on the channel width:

$$
\left|\phi_{\max }\right| \approx \frac{\sqrt{3}}{\delta \lambda^{2}}
$$

The ASKAP frequency resolution of $19 \mathrm{kHz}$ provides $\left|\phi_{\max }\right|$ more than sufficient to sample the full range of RM experienced in the Galactic interstellar medium. The following table lists the Rotation Measure synthesis properties of ASKAP.

\begin{tabular}{cccc}
\hline $\begin{array}{c}\nu_{\text {low }} \\
\mathrm{MHz}\end{array}$ & $\begin{array}{c}\nu_{\text {high }} \\
\mathrm{MHz}\end{array}$ & $\begin{array}{c}\delta \phi \\
\mathrm{rad} / \mathrm{m}^{2}\end{array}$ & $\begin{array}{c}\text { max-scale } \\
\mathrm{rad} / \mathrm{m}^{2}\end{array}$ \\
\hline 800 & 1100 & 37 & 42 \\
800 & 1700 & 10 & 102 \\
\hline
\end{tabular}

The initial instantaneous $300 \mathrm{MHz}$ bandwidth of ASKAP provides only limited capacity for Rotation Measure synthesis science. However this picture changes by synthesizing the full $900 \mathrm{MHz}$ RF band pass, by for example multiple observations with the centre frequency tuned to cover the full bandpass. In order to resolve Faraday thick structures along the line of sight, $\lambda_{\text {min }}^{2}$ must be smaller than $\Delta \lambda^{2}$, which is obtained over the full $900 \mathrm{MHz}$ RF band.

\subsection{The Inter-Galactic Medium}

Beyond individual galaxies and clusters, all of "empty" space may be magnetized, either by outflows from galaxies, by relic lobes of radio galaxies, or as part of the largescale structure of the cosmic web. Such a field has not yet been detected, but its role as the likely seed field for galaxies and clusters, plus the prospect that the IGM field might trace and regulate structure formation in the early Universe, places considerable importance on its discovery. Once the magnetic field of the IGM is detected, a measurement of the characteristic size scales of its fluctuations can allow us to differentiate between the wide variety of mechanisms proposed for magnetic field generation in the IGM (see Gaensler et al. 2004 and references therein). While RMs of distant EGS are potentially a useful probe of the IGM, to date there has been no detection of magnetic fields in the IGM; current upper limits on the strength of any such field are model dependent, but suggest $\left|B_{\mathrm{IGM}}\right| \lesssim 10^{-8}-10^{-9} \mathrm{G}$ (Kronberg 1994, Blasi et al. 1999).

With a sufficiently large RM sample, this all-pervading cosmic magnetic field may eventually be identified. Just as the correlation function of galaxies yields the power spectrum of matter, the analogous correlation function of this RM distribution can provide the magnetic power spectrum of the IGM as a function of cosmic epoch and 
over a wide range of spatial scales. Such measurements will allow us to develop a detailed model of the magnetic field geometry of the IGM and of the overall Universe.

For Faraday rotation occurring at a redshift $z$, the observed RM is reduced by a factor $(1+z)^{2}$ over its intrinsic value. However, a simplistic assumption is that the comoving magnetic flux and electron density are both constant, so that electron density evolves as $(1+z)^{3}$, while the magnetic field evolves as $(1+z)^{2}$ (e.g. Widrow 2002). In this case, we expect the observed RM to be proportional to $(1+z)^{3}$. While this is a naive calculation, it demonstrates the general principle that RM signatures from distant objects can be quite large, even if the foreground IGM has a relatively small magnetic field. More robust treatments are presented by Kolatt (1998) and Blasi et al. (1999), who demonstrate in detail how the statistics of RM measurements as a function of redshift can be used to remove the foreground Galactic contribution, and then to extract the strength and power spectrum of magnetic fields in the IGM.

These studies require a very large sample of RMs from sources at known redshifts, and thus are probably more likely to be addressed with the SKA than with ASKAP. However, recent results such as those shown in Figure 29 show that at least in certain specific regions, it may be possible to identify very weak magnetic fields over scales $>1 \mathrm{Mpc}$ with more modest RM samples. If ASKAP can be expanded so that it can push down even further in continuum sensitivity, its large sky coverage and dense RM sampling might make the first such studies possible, especially for fields which have already been well-studied at other wavelengths. In any case, ASKAP data will act as a crucial pathfinder for SKA studies of the IGM, by identifying the likely source statistics and survey yields, and by allowing us to quantify the accuracy with which foreground RMs can be modelled and then removed.

\subsection{Technical Specifications for Polarimetry with ASKAP}

The accuracy of the RMs from a polarization survey is set by the total bandwidth and the signal-to-noise. For an RM grid survey that uses a $300 \mathrm{MHz}$ bandwidth centred on $1.4 \mathrm{GHz}$, a signal-to-noise ratio of 10 will provide an uncertainty in $\mathrm{RM}$ of $\pm 5 \mathrm{rad} \mathrm{m}^{-2}$. This level of precision is needed to detect the small levels of Faraday rotation expected at high Galactic latitudes, but higher precision is problematic due to systematic effects such as ionospheric Faraday rotation. The maximum RM to which a survey is sensitive is set by the width of individual channels: for 1-MHz channels (i.e., 300 channels across the band), the maximal signal will be $|\mathrm{RM}| \approx 22000 \mathrm{rad} \mathrm{m}^{-2}$, which will be more than enough for most experiments.

For typical AGN spectra and a fixed total bandwidth, the precision in RM is proportional to $\nu^{3.5}$, so that higher frequency surveys give much larger RM uncertainties. Conversely, depolarization effects become severe at low frequencies, such that although $\mathrm{RM}$ measurements are accurate, most sources are not polarized. Considering both these effects, a centre frequency of $1.4 \mathrm{GHz}$ seems to be a "sweet spot" for EGS RM surveys.

An extragalactic RM survey does not set strong constraints on the array configuration for ASKAP, since the target fields will primarily be compact sources. While there will be minimal source confusion in Stokes $Q$ and $U$, accurate measures of fractional polarizations require baselines of about $6 \mathrm{~km}$ due to confusion limits in Stokes $I$ (see Section 4.1.1). However, angular scales of $\approx 20^{\prime \prime}-30^{\prime \prime}$ are well matched to the source dimensions; at higher resolution, emission from some sources will to be resolved 

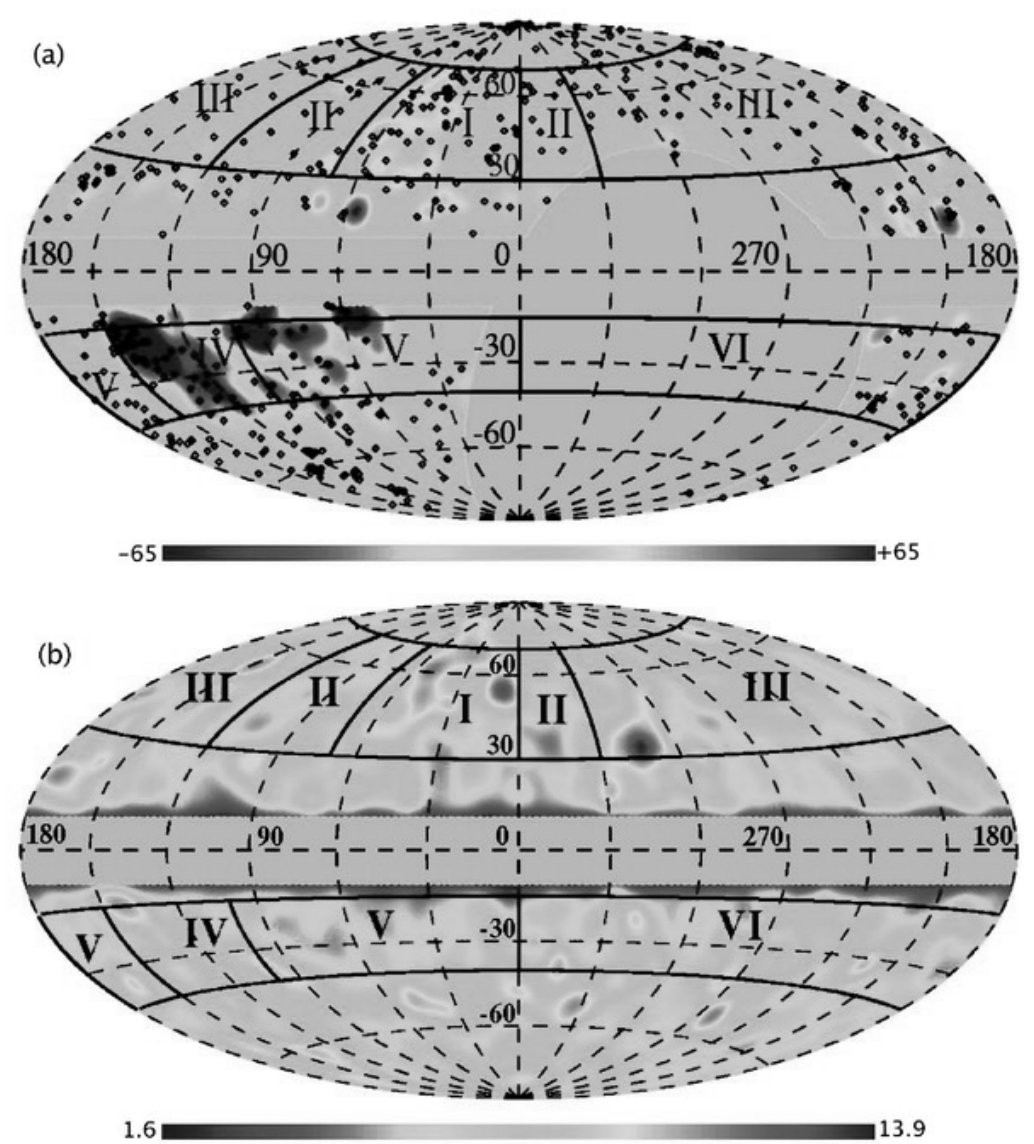

Fig. 29 (a) An all-sky plot of the smoothed distribution of EGS RMs. The black squares show the positions of individual EGS RM measurements. The colour map shows RM variations, smoothed to $7^{\circ}$ resolution in an Aitoff projection of Galactic coordinates. The grescale range from white to black signifies RMs ranging from -65 to $+65 \mathrm{rad} \mathrm{m}^{-2}$. (b) The 2MASS galaxy column density similarly smoothed to $7^{\circ}$ resolution. The Hercules supercluster, lying in the high density region labelled I, shows an RM distribution which is possibly correlated with the galaxy distribution. This suggests the presence of a large-scale coherent magnetic field in this supercluster, with strength $\sim 0.3 \mu \mathrm{G}$ on scales $\sim 0.5 \mathrm{Mpc}$. Figure from Xu et al. (2006) with the kind permission of the authors.

out. Therefore a concentration of collecting area within a central $\sim 1 \mathrm{~km}$ core with significant collecting area extending to baselines of about $6 \mathrm{~km}$ is ideal.

For accurate polarization calibration, good parallactic angle coverage of each field is highly desirable. Thus even if the snapshot $u v$ coverage of ASKAP is very good, each image needs to be constructed from multiple cuts. However, any long-term source variability in polarization will make it difficult to measure RMs. Thus the multiple cuts on each field are best spread over a single 12-hour track, perhaps appropriate for initial observing in H I galaxy surveys. Continuum polarization observations are best carried out at night, and continuous GPS monitoring of the total electron content will be needed to correct for ionospheric Faraday rotation. Since most faint EGS will have 
a fractional linear polarization of $\sim 10 \%$, the final images will require a polarization purity of at least $-20 \mathrm{~dB}$ across the entire field.

The survey data that will need to emerge from the reduction pipeline will be all-sky maps of Stokes $I, Q, U$ and $V$ in 1-MHz channels, corresponding to a total size for the final data cube of $\sim 12$ TB. However, the high-level end product will be much smaller, simply consisting of a catalogue of position, flux, fractional polarization and RM for each of the sources in the RM grid (total catalogue size $\sim 15 \mathrm{MB}$ ).

To probe the turbulent magnetoionic medium in the Galactic halo and disk-halo interface will require a complementary all-sky survey of diffuse polarization utilising the full frequency range available to ASKAP. From Wolleben et al. (2006) we know that polarised structure exists at $1.4 \mathrm{GHz}$ down to at least the $\sim 12 \mathrm{mK}$ sensitivity limit of their survey. Average rotation measure fluctuations in the Milky Way are expected to be a few tens of $\mathrm{rad} \mathrm{m}^{-2}$. To ensure that we do not suffer from bandwidth depolarization in regions of high rotation measure found in the Galactic plane, we need modest channel widths of $\sim 4 \mathrm{MHz}$. Finally, in order to correctly interpret rotation measures it is crucial that we combine the interferometric survey data with total power information from a single dish. This survey, therefore, will require a survey of comparable sensitivity and frequency coverage conducted with the Parkes $64 \mathrm{~m}$ telescope. 
5 Galactic and Magellanic Science

Lead authors: N. McClure-Griffiths, G. Joncas

Contributing author: D. Matthews

\subsection{Summary}

The rebirth of observational studies of the Milky Way and Magellanic System over the past decade has raised new and profound questions about the evolution of the interstellar medium (ISM). The community has transitioned from studying small-scale aspects of the ISM to a more wholistic approach, which seeks to combine information about a variety of ISM phases with information about magnetic fields. With ASKAP we can make significant and unique inroads into understanding the evolution of the ISM and through that the evolution of the Milky Way. These are crucial steps along the path to understanding the evolution of galaxies.

The Milky Way and Magellanic System because of their very large sky coverage can only be observed in survey mode. As such, they present themselves as ideal targets for ASKAP. Here we discuss how ASKAP can be used to achieve superb advances in our understanding of the evolution of the Milky Way, its ISM and its magnetic field. To achieve these advances we propose several large-scale projects, which include: accounting for and studying the structure of all $\mathrm{H}$ I associated with the Milky Way and Magellanic System to unprecedented column-density limits and angular resolution; constraining the large-scale Galactic magnetic field in the inner Galaxy with in situ measurements of $\mathrm{H}$ I Zeeman splitting; exploring the growth of molecular clouds using diffuse $\mathrm{OH}$ mapping of the Galactic plane; and probing the turbulent magneto-ionic medium of the Galactic halo with diffuse continuum polarization mapping of the whole sky. The unifying technological requirement for all aspects of the Galactic and Magellanic science presented herein is the demand for low-surface-brightness sensitivities achieved by compact configurations.

\subsection{The Milky Way as a Laboratory for Galaxy Evolution}

Galaxy evolution is one of the great puzzles in current astrophysics, incorporating how galaxies assemble and evolve from the beginning of the Universe to the present day. Unfortunately, our knowledge of the fundamental processes of galaxy evolution is blocked because we do not understand the evolutionary cycle of interstellar matter in our own Galaxy. We know that the life cycle of the Milky Way, and of most other spiral galaxies, involves a constant process of stars ejecting matter and energy into the interstellar mix, and new stars condensing from this matter. Somehow matter makes the transition from hot, ionised stellar by-products to become the cold molecular clouds from which stars are formed. The circulation of matter between the Galaxy's disk and its surrounding halo further complicates this cycle. ISM studies in our Galaxy probe the evolutionary cycle of matter with sensitivity and resolution unattainable in external galaxies; it is only in the Milky Way that we can observe the evolution of the interstellar medium on scales ranging from sub-parsec to kiloparsecs. Furthermore, the evolution of the Galaxy is controlled by the Galactic and local magnetic fields, yet these components 
are largely unknown. The Milky Way is thus an ideal laboratory for studying galaxy evolution.

In recent years there has been a renaissance in Galactic ISM surveys. This has largely been driven by advances with the Canadian and Southern Galactic Plane Surveys, which used a combination of single-antenna and aperture-synthesis telescopes to map the Galactic plane in $\mathrm{HI}$ and polarized continuum emission at an unprecedented resolution of $\sim 1^{\prime}$. From these surveys have followed a number of Galactic Plane projects at comparable or better resolution such as the FCRAO and Boston University surveys of ${ }^{12} \mathrm{CO}$ and ${ }^{13} \mathrm{CO}$ and the Spitzer GLIMPSE survey of infrared emission. These surveys have revised the way we think about ISM studies, revealing structure on all size scales, a remarkable agreement between ISM phases, and a wide variety of new polarization structures. More recently there has been a push to improve the all-sky $\mathrm{H} \mathrm{I}$ and radio continuum atlases from the $\sim 1^{\circ}$ resolution of the past 15 years to $\sim 15^{\prime}$.

The future of Galactic and Magellanic ISM studies lies in low-surface-brightness observations. For the Milky Way, the drive is to extend the arc-minute resolution work pioneered in the Galactic plane to the Galactic halo and the disk-halo interface. These regimes will allow us to better understand how the Milky Way interacts with its environment, how matter is expelled from the disk and how the Galaxy has evolved. Here we describe a number of projects which will use ASKAP to revolutionise our understanding of the Milky Way and its ISM. The observational tools for these projects include $\mathrm{H}$ I and $\mathrm{OH}$ emission, $\mathrm{H}$ г Zeeman splitting and diffuse polarized emission.

\subsection{H i Associated with the Milky Way and Magellanic System}

\subsubsection{High Velocity Clouds and the Galactic Halo}

High and intermediate velocity clouds (HVCs and IVCs) are believed to play important roles in both the formation and the evolution of the Galaxy. Some HVCs may be related to the Galactic Fountain; some are tidal debris connected to the Magellanic Stream (Putman et al. 2003) or other satellites; some may be infalling intergalactic gas, and some may be associated with Dark Matter halos and be the remnants of the formation of the Local Group. The structure and distribution of high velocity gas probes tidal streams and the building blocks of galaxies, providing critical information on the evolution of the Milky Way system. One problem that has long plagued HVC studies is the almost complete lack of distances to these objects, which has traditionally sparked debates about whether HVCs are Galactic or extragalactic. Although the distance problem is lessening somewhat with increased number of distance measures using absorption towards halo stars, the problem remains. Regardless, the consensus now is that there are probably a variety of HVC origins.

Nearby HVCs can be used as probes of the thermal and density structure of the Galactic halo. HVCs have, in general, a high velocity relative to their ambient medium which results in a ram-pressure interaction between the cloud and medium. Recent $\mathrm{H}$ I observations have indeed shown that a significant fraction of the HVC population has head-tail or bow-shock structure (Brüns et al. 2000, 2001). By comparing the observed structures and thermal distributions within them to numerical simulations it is possible to determine basic physical parameters of the Galactic halo such as density, pressure, and temperature. In situations where the density and pressure of the ambient medium 
are known by independent methods, the problem can be turned around to determine the distance to the observed clouds (Peek et al. 2007).

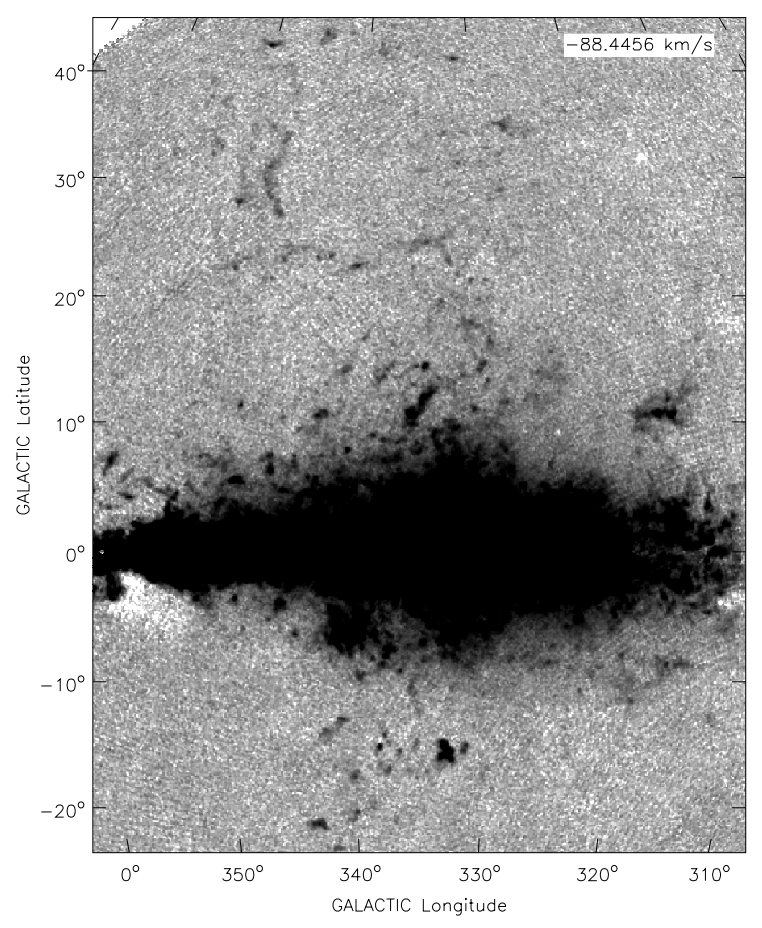

Fig. $30 \mathrm{H}$ I emission from the Galactic All-Sky Survey at $-88.5 \mathrm{~km} \mathrm{~s}^{-1}$ showing the tenuous structure connecting the clouds of Complex L to the Galactic Plane (McClure-Griffiths et al., in preparation).

The recent Galactic All-Sky Survey (GASS; McClure-Griffiths et al., in preparation) has imaged the sky south of declination zero at $15^{\prime}$ resolution to a $1 \sigma$ brightness temperature limit of $70 \mathrm{mK}$ per $\mathrm{km} \mathrm{s}^{-1}$, or a column density limit of $N_{H} \approx$ $8 \times 10^{17} \mathrm{~cm}^{-2}$ over $20 \mathrm{~km} \mathrm{~s}^{-1}$. The angular resolution of this survey offers a factor four improvement over previous all-sky surveys (IAR-LDS) and the $1 \mathrm{~km} \mathrm{~s}^{-1}$ velocity resolution offers a factor of 15 improvement in spectral resolution over HIPASS. With these improvements we are beginning to resolve the structure of some of the mid-sized HVCs. However, we do not yet have the sensitivity or resolution necessary to study the detailed physical and thermal structure over a large number of HVCs. It seems that the structure that we do see is just the tip of the iceberg.

GASS is also revealing a wealth of tenuous filaments connecting HVCs to the Galactic Plane at column densities of $N_{H} \sim 10^{18} \mathrm{~cm}^{-2}$. An example is in Figure 30, which shows that the HVCs in Complex L are interconnected by a thin, low column-density filament and that this filament appears to extend to the Galactic disk. Although Complex L is clearly Galactic, filaments of more distant HVCs may be related to the "cos- 
mic web" predicted in cosmological simulations. Recent observations of the M31-M33 system (Braun \& Thilker 2004) have revealed the local analogue of the cosmic web, showing that very low column-density gas $\left(N_{H} \sim 10^{16-17} \mathrm{~cm}^{-2}\right)$ connects the two galaxies. The current generation of Milky Way H i surveys do not reach the column density limits needed to explore this fascinating web of structure in the entirety of the Local Group. An H i survey with ASKAP to a brightness limit of $100 \mathrm{mK}$ per $\mathrm{km} \mathrm{s}^{-1}$ at $2^{\prime}$ resolution could be smoothed to reach an unprecedented column density limit of $N_{H} \sim 2 \times 10^{16} \mathrm{~cm}^{-2}$ at $15^{\prime}$ resolution over the entire sky. This survey would reveal the majority of the cosmic web in the Local Group and provide important constraints for models of the formation of structure in the Local Group.

\subsubsection{Disk-Halo Interaction}

We know that the evolution of the Milky Way is significantly impacted by the twoway flow of gas and energy between the disk and halo. Although we can now detect enormous amounts of structure in the atomic component of the Milky Way halo, we are far from understanding its origin and its relationship to the processes that dominate in the disk. There are a number of important unanswered questions about the transfer of matter between the disk and halo. For example, the range of metallicities and stellar ages observed in the Milky Way suggests that it must continually receive fresh fuel for star formation, but how does the Galaxy receive the fresh fuel for star formation? What happens to matter expelled from the disk? Does it cool and return to disk and if so how long does the process take? Alternately, does expelled matter remain in the halo? Finally, what are the physical conditions of the halo? Our ability to answer these questions is significantly hampered by our inability to image the detailed physical structure of the $\mathrm{HI}$ in the halo.

Recent work by Lockman (2002) and Ford et al. (2008, in preparation) has shown that a significant amount of mass in the lower halo is contained in small clumps of $\mathrm{HI}$, such as those shown in Figure 31. The nature and origin of these cloudlets, which participate in Galactic rotation and are only tens of parsecs in size, are at present unknown. Do they originate from outflow from the Galactic disk or are they related to inflow from extragalactic space? The one thing that we do know is that small, mostly unresolved, cloudlets are ubiquitous throughout the inner Galaxy (see Figure 31).

With the present generation of surveys at $15^{\prime}$ resolution it is not possible to explore the thermal and physical structure of these cloudlets, which may contain information about their origin, motion and evolution. Follow-up observations on several individual cloudlets, such as those shown in the right panel of Figure 31, show a tantalising glimpse of possible head-tail structure and other evidence of interaction. These observations may offer the opportunity to resolve directional ambiguity with these clouds. In general we can measure only the absolute value of the $z$ component of the cloud velocity with respect to the Galactic Plane, i.e. we cannot determine whether the clouds are moving towards or away from the Galactic Plane. Finding head-tail structure in the clouds can help resolve this ambiguity by showing the direction of motion. At present these follow-up observations are prohibitive, requiring hundreds of hours with the ATCA to reach $100 \mathrm{mK}$ at arc-minute scales.

Ideally we would like to apply the same techniques that we use with HVCs to halo cloudlets and use the clouds' structure to probe the temperature and density structure of the lower halo. The temperature and density structure of the Galaxy's lower halo is very poorly constrained with values for $n T$ ranging from 10 to $1000 \mathrm{~K} \mathrm{~cm}^{-3}$. Although 


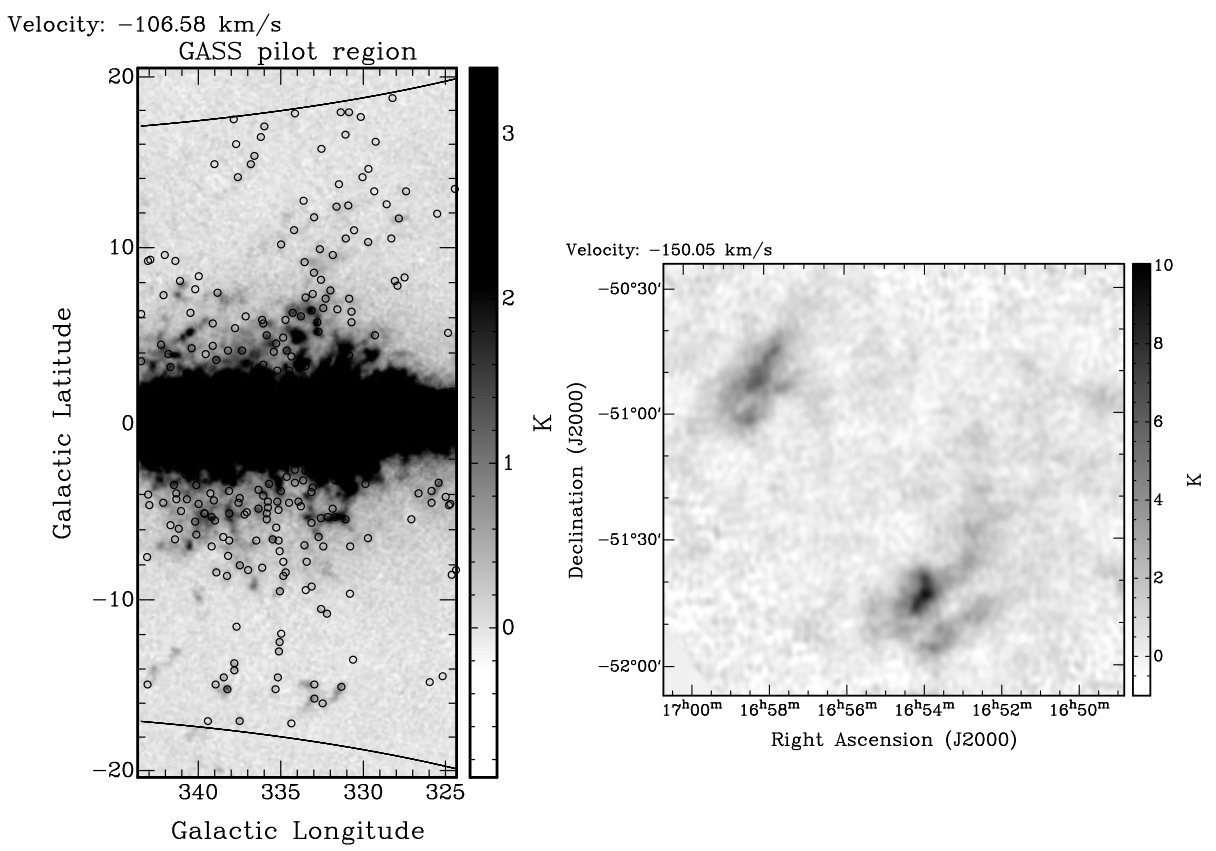

Fig. 31 Left: H I emission at $15^{\prime}$ resolution from GASS at an LSR velocity of $-107 \mathrm{kms}^{-1}$. The greyscale shows a number of small HI halo cloudlets sitting above and below the Galactic plane and is linear in brightness temperature from -0.9 to $3.2 \mathrm{~K}$. Overlaid on the greyscale are circles marking the positions of halo cloudlets detected at other velocities within the observed region (Ford et al., 2008, in preparation). These cloudlets are hypothesised to be the result of explosive events in the Galactic disk and their distribution about the Galaxy may shed some light on their origin. Right: High-resolution $\left(\sim 2^{\prime}\right) \mathrm{H} \mathrm{I}$ image of two halo cloudlets made with the ATCA and Parkes. This image shows that clouds, which appear unresolved at $15^{\prime}$ resolution, have complex structures suggesting interaction with the ambient medium. The tantalising glimpse of possible head-tail structure in these clouds could provide information about the clouds' direction of motion (Ford et al., 2008, in preparation).

diffuse X-ray emission and ultraviolet spectral lines, such as OvI, can help constrain the properties of the hot ionised medium in the lower halo, the filling factor of the warm neutral medium and its physical properties are largely unknown. Halo cloudlets can provide unique insight into this important transition region from Galactic disk to halo.

\subsubsection{Turbulence in the Milky Way}

There is little doubt that interstellar turbulence plays a significant role in the structure, kinematics and global evolution of the ISM (see review papers by Elmegreen \& Scalo 2004 and Scalo \& Elmegreen 2004). Observationally, the signature of turbulence has been detected in molecular clouds (Brunt 2003 and references therein), H II regions (Joncas 1999) and H I features (Miville-Deschênes et al. 2003, Armstrong et al. 1995, Stanimirovic \& Lazarian 2001) on scales ranging from AU to kpc. However, turbulence is still far from understood, especially given that magnetic fields are present and almost certainly affect its properties. Many numerical simulations have 
been done or are ungoing to alleviate this situation but they neglect many constraints relevant to the ISM that would make them realistic (Avila-Reese \& Vazquez-Semadeni 2001, Monaghan 2002, Audit \& Hennebelle 2005). These simulations can be improved markedly by adding observational constraints provided by a better characterization of the statistical properties of the interstellar gas over a large range of spatial scales. This improvement would also help in addressing a number of outstanding problems related to the description of the energy injection, dissipation and distribution over a large range of spatial scales such as: What are the sources of galactic turbulence? How does the energy cascade take place? How does it dissipate at small scales or is intermittency dominating? Is it Kolmogorov-like on all scales? What is the role of the magnetic field and how does the environment affect the strength of the turbulence?

Current observational studies of turbulence over large areas are limited to arcminute scales and mostly within the Galactic Plane. The Galactic Plane, however, is a unique environment where turbulence can be driven by star formation and other active processes. Recent observational studies of very small areas out of the plane have confirmed the predicted abundance of faint, very small H I structures (Stanimirovic \& Heiles 2005, Minter et al. 2005, Braun \& Kanekar 2005). These pointed observations, however, need to be superseded by coverage of large areas to allow characterisation of the spatial power spectrum of $\mathrm{H}$ i on as many scales as possible. We therefore propose deep H I mapping of a large area at high Galactic latitude to best probe turbulence on spatial scales ranging from thousands of AU to hundreds of parsecs. High latitude regions, where the turbulence presumably draws its energy only from the gas flow, are ideal for this study.

The fast mapping speed of ASKAP would uniquely enable it to cover a sufficiently large area to probe statistics at sub-arc-minute resolution. The proposed experiment would be a deep H I data cube of a single $50 \mathrm{deg}^{2}$ area at high Galactic Latitude with an angular resolution of $\sim 30^{\prime \prime}$. Extrapolating from lower resolution studies of the $\mathrm{H}$ I spatial power spectrum, we know that the brightness temperature of $\mathrm{H}$ I continues to decrease to sub-Kelvin levels at sub-arc-minute angular scales. It will therefore be necessary to map the region to a sensitivity limit of $\sim 200 \mathrm{mK}$ in $1 \mathrm{~km} \mathrm{~s}^{-1}$ channels. A resolution of $\sim 30^{\prime \prime}$ translates to a physical resolution of $2000 \mathrm{AU}$ at a distance of $100 \mathrm{pc}$ and $0.3 \mathrm{pc}$ at a distance of $2 \mathrm{kpc}$, which covers the range of distances that would be sampled by this study. A panoply of statistical descriptors exist that can be used to probe turbulence including: principal component analysis, probability density functions, power spectra, genus analysis, spectral correlation functions, high order correlation functions, and the wavelet transform modulus maxima method.

Turbulence holds the key to many crucial galactic processes such as star formation (Li et al. 2003, Larson 2003), cloud structure and dynamics (Scalo 1990) and the evolution of the different phases of the ISM. Its characterization on Galactic scales is necessary to evaluate its impact on Galactic evolution (see models by Gazol-Patiño \& Passot 1999 and references therein). The combination of excellent data with the above mentioned tools holds the key to determining if the numerical calculations, which are abundant in the literature, reproduce interstellar turbulence correctly.

\subsubsection{HI in the Magellanic System}

At a distance of $65 \mathrm{kpc}$, the gas-rich irregular dwarf galaxies, the Large and Small Magellanic Clouds, form an interacting triad with the Milky Way. Located at approximately 
$-70^{\circ}$ declination an uninterrupted view of almost the entire system is achievable. Evidence of the three-way interaction is in the form of the three tidal features associated with the system (Putman 2000). The Bridge forms an apparently contiguous H I filament connecting the SMC and LMC. A very extended halo of H I gas, the Magellanic Stream, trails the clouds and subtends an area over $1000 \mathrm{deg}^{2}$. The Leading Arm is the tidal counterpart of the Magellanic Stream, preceding the motion of the clouds around the Galaxy.

Observations of the entire Magellanic System have only been obtained with the relatively low resolution of the Parkes telescope $\left(\sim 14^{\prime}\right)$. High-resolution observations of the higher H I column-density regions of the LMC, SMC and Bridge exist with resolutions down to $\sim 1^{\prime}$ and sensitivities of $10^{18} \mathrm{~cm}^{-2}$. Recently the ATCA was used to map the Magellanic Stream from its origin to $0^{\circ}$ declination achieving resolutions of $\sim 7^{\prime}$ and column densities down to $\sim 10^{19} \mathrm{~cm}^{-2}$.

In order to fully understand the structure, formation processes and turbulence characteristics of the entire Magellanic System, higher resolution images are required. Such data would enable the study of the turbulent ISM, and signatures of the interactions between the galaxies such as head-tail features, the compression of the LMC disk and the stripping of matter by the Galactic $\mathrm{H}$ I halo. An understanding of the extent of $\mathrm{H}$ I around the System to better sensitivity would increase the sophistication of numerical simulations.

The large field of view of ASKAP makes it ideal for an investigation of the entire Magellanic System (over $2500 \mathrm{deg}^{2}$ ). However deep imaging of the low-column-density extended $\mathrm{H}_{\mathrm{I}}$ would be required to obtain the target sensitivities $\left(\sim 10^{17} \mathrm{~cm}^{-2}\right)$. If it proves impossible to reach these sensitivity limits, studies of the Magellanic Stream and Leading Arm could still greatly benefit from ASKAP. An area of $\sim 1000 \mathrm{deg}^{2}$ could be observed to $\sim 1^{\prime}$ resolution at a column density limit of $10^{18} \mathrm{~cm}^{-2}$. Such an investigation would reveal structures down to scales not yet observed, increasing the knowledge base on the organisation of structure in the Stream. Comparisons with existing and new results from other areas of the System would create a much more complete picture of the Magellanic ISM. The improved spatial resolution would also enable searches for morphological signature of $\mathrm{H}$ i infall into the Galactic Halo. A deep survey of the eastern Magellanic Bridge $\left(\sim 50 \mathrm{deg}^{2}\right)$ would complement existing ISM turbulence studies of the western Bridge.

\subsubsection{An All-Sky Galactic Hi survey}

ASKAP is undoubtedly a survey instrument. The Milky Way and Magellanic Systems, because they fully surround us, demand large area surveys. An all-sky Galactic H I survey with ASKAP would build on GASS and current Magellanic surveys to provide a full census of all $\mathrm{H}$ I associated with the Milky Way and the Magellanic System at arc-minute resolution. This survey would provide essential information about the physical and thermal structure of HVCs and their interaction with the halo; the role and origin of halo cloudlets and the physical structure of the Magellanic Stream and Leading Arm.

In order to study Galactic and Magellanic H I we require an all sky survey with $\sim 2^{\prime}$ angular resolution and $\sim 1 \mathrm{~km} \mathrm{~s}^{-1}$ velocity resolution $(5 \mathrm{kHz})$. HVCs and the structures that connect them are typically very low column density, $N_{H} \sim 10^{18} \mathrm{~cm}^{-2}$. Over a typical velocity width of $\sim 20 \mathrm{~km} \mathrm{~s}^{-1}$, this requires rms sensitivities at the $100 \mathrm{mK}$ level or below. Galactic H I at high latitudes is also very low surface brightness. 
Ford et al (in preparation) have found that the distribution of halo cloudlets peaks at $\sim 0.5 \mathrm{~K}$. In addition to low surface brightness sensitivity, the halo structures of interest can cover large areas of the sky and therefore require good short baseline coverage.

To achieve the goals of these projects we need to reach a low surface brightness temperature sensitivity limit of $\sim 100 \mathrm{mK}$ over a $\sim 2^{\prime}$ synthesised beam. The important figure of merit here is the surface-brightness-limited mapping speed given in Chapter 1. From equation 3 it is clear that the filling factor of the array, $f$, is crucial to achieving a reasonable mapping speed for surface-brightness sensitivity limited surveys. We rewrite equation 4 in the form

$$
f=\frac{A \epsilon_{a} N}{\pi / 4 L^{2}},
$$

where $L$ is the maximum baseline length. For surface-brightness sensitivity limited surveys the mapping speed goes as $L^{-4}$ so that for a fixed collecting area the surface brightness sensitivity achieved is strongly dependent on the resolution of the survey. Therefore, in order to survey the entire southern sky to a $100 \mathrm{mK}$ sensitivity limit in a reasonable $(\sim 1 \mathrm{yr})$ time, ASKAP would need to be configured with a maximum baseline of $\sim 500 \mathrm{~m}$, or equivalently the filling factor needs to be $>0.01$.

An additional consideration for this survey is the need to include single-dish data for sensitivity to structure on the largest scales. Fortunately the Galactic All-Sky Survey already exists with comparable sensitivity and spectral resolution to the proposed survey, and can be combined with the ASKAP survey.

\subsection{Magnetic Fields in the Galaxy: H i Spectropolarimetry}

One of the key science drivers for the SKA is understanding the magnetic universe. For the Milky Way, rotation measures of extragalactic point sources as described in Chapter 4 will yield information about the line-of-sight averaged magnetic field weighted to regions of ionised gas. To fully understand the Galactic field and its effect on the evolution of the Galaxy, we also require in situ measurements of the field throughout the Galaxy. One technique for obtaining in situ measurements is Zeeman splitting of the H I line (e.g. Heiles \& Troland 2003, 2005). Using the kinematic information in the $\mathrm{H}$ I line and its relationship with Galactic distance gives information about the third dimension that is not available with rotation measures.

There are three types of measurements that can be made with H I spectropolarimetry: H I Zeeman measurements of absorption towards background continuum sources, H I Zeeman measurements of emission at the tangent point and $\mathrm{HI}$ absorption of the polarized continuum background. Of these, H I Zeeman measurements of emission at the tangent point is the most novel and has the potential to provide the most information about the magnetic field strength over a very large region of the inner Galaxy. Measuring H I Zeeman is not trivial; the intrinsic velocity separation induced by Zeeman splitting of the $\mathrm{HI}$ line is extremely narrow, only $2.8 \mathrm{~Hz}$ per $\mu \mathrm{G}$. To detect Zeeman splitting we measure the difference between the right and left circular polarizations, Stokes V, which is proportional to the velocity derivative of the Stokes I profile, $V=2.8 B_{\text {los }} d I_{\nu} / d \nu \mathrm{Hz} \mu \mathrm{G}^{-1}$.

Each line of sight in the Milky Way interior to the Solar circle passes a point where its projection on the Galactic plane is tangent to a circular orbit around the Galactic centre and the distance is geometrically determined. This locus of tangent points is shown with a thick white line in Figure 32, which is a model of the Galaxy overlaid 


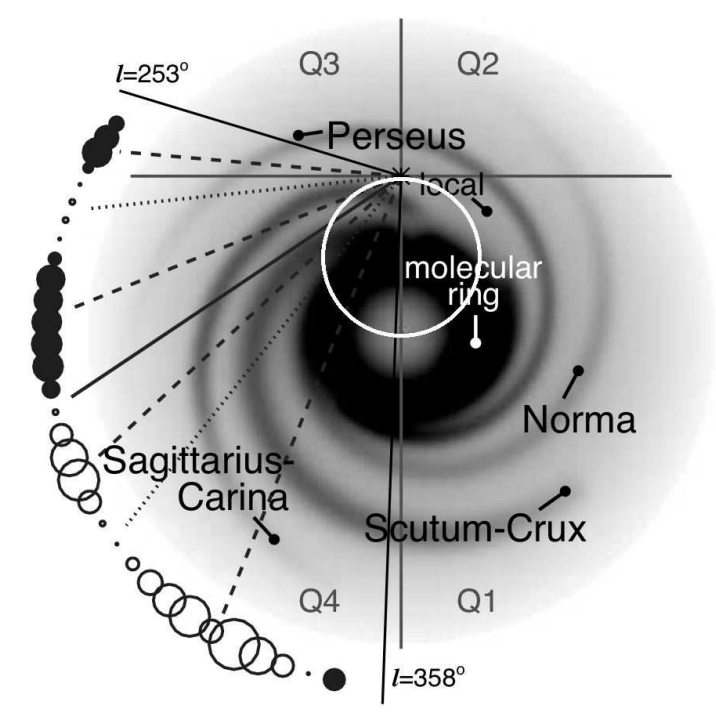

Fig. 32 The Cordes \& Lazio (2002) model of the Galactic spiral structure overlaid with rotation measures reproduced from Brown et al. (2007). The thick white line shows the locus of tangent points where in situ magnetic field measurements can be obtained by H I Zeeman measurements at the terminal velocity.

with recent rotation measures from Brown et al. (2007). At the tangent point the H I emission reaches its most extreme velocity, the terminal velocity, beyond which the $\mathrm{H}$ I profile drops off steeply. Because of the steep drop beyond the terminal velocity it is possible to measure the Zeeman effect. This method will yield an in situ measurement of the magnetic field at every point along the locus of tangent points, which will tie down the Galactic magnetic field models in a way that is not possible with any other measurement.

\subsubsection{A Galactic Plane Survey of H I Zeeman in Emission}

The ideal survey to conduct this tangent point Zeeman experiment would cover as much of the area $-90^{\circ} \leq l \leq+90^{\circ}$ as possible. A small, but significant latitude coverage of $\sim 5^{\circ}$ is needed to ensure that our measurements reflect the global Milky Way magnetic field, rather than being subject to individual clouds. We therefore propose a survey of the area $270^{\circ} \leq l \leq 45^{\circ},|b| \leq 2.5^{\circ}: \sim 700 \mathrm{deg}^{2}$. For a typical magnetic field strength of $\sim 5 \mu \mathrm{G}$ and a velocity width of $5 \mathrm{~km} \mathrm{~s}^{-1}$, we expect the amplitude of the Stokes $\mathrm{V}$ spectrum to be $70 \mathrm{mK}$. We require that the derivative of the line be very well sampled in velocity space, so that for a typical derivative line width of $5 \mathrm{~km} \mathrm{~s}^{-1}$, we require $\Delta v \approx 0.25 \mathrm{~km} \mathrm{~s}^{-1}$ or $\Delta \nu \approx 1 \mathrm{kHz}$. This survey requires a $1 \sigma$ sensitivity limit of $\sim 20$ $\mathrm{mK}$ in each polarization. In order to reach these low surface brightness limits we once again require that the telescope have a high filling factor. Because resolution is not a stringent demand on this project, an ultra-compact configuration with most of the collecting area inside a maximum baseline of $\sim 200 \mathrm{~m}$ would be preferred. Measuring $\mathrm{H} \mathrm{I}$ Zeeman in emission requires both low sensitivity limits and good polarization properties of the telescope. Interferometers are well suited to this experiment because polarization 
calibration can be achieved to better accuracy than with a single dish. With such a compact configuration ASKAP would be uniquely positioned to measure the magnetic field along the tangent point and contribute to directly constraining models for the Milky Way magnetic field.

The H I spectropolarimetric dataset will have several other important uses. It can be used to obtain measurements of H I Zeeman in absorption towards continuum sources in the Galactic Plane. This will provide hundreds of measurements of the magnetic field contained in cold $\mathrm{H}$ I clouds. We know that the magnetic field is important in determining the structure and evolution of molecular clouds, which must evolve out of cold atomic clouds. The dataset can also be used to measure H I absorption against the Galactic polarized background (Dickey 1997), which will give kinematic distances to the polarized background throughout the inner Galaxy.

5.5 Probing the Evolution of Cold Gas in the Milky Way: OH Emission and H I Self-Absorption

A significant hurdle in our understanding of the evolution of the ISM and the formation of stars is how molecular clouds form from cold atomic gas. The process by which a previously-atomic cloud becomes molecular is believed to involve compression of the cloud so that the column density of the atomic material becomes significant enough to self-shield and thereby reduce the photo-dissociation of $\mathrm{H}_{2}$ (Elmegreen 1991). The timescale required for this process is predicted by various theoretical calculations but there are virtually no observational constraints. Recent work by Goldsmith \& Li (2005) and Goldsmith et al. (2007) has shown that measurements of the residual cold atomic gas fraction within a molecular cloud may provide a means for dating the molecular cloud itself. The formation time for molecular clouds and the star formation timescale within a given cloud have important implications for the global star formation rate in galaxies and the evolution of the Galaxy. As a molecular cloud condenses there is a time-dependent relationship of the molecular to atomic fraction. This molecularcloud dating technique, however, requires knowledge about the fraction of atomic to molecular gas as well as the temperature and density within the cloud. To obtain information about the molecular content of the gas, and to constrain the temperature and density of the cloud it is possible to use diffuse $\mathrm{OH}$ emission.

It has long been recognised that $\mathrm{OH}$ emission is a good tracer of cold gas in the Galaxy (e.g. Dickey et al. 1981). H I is a poor tracer in cold dense clouds because its thermal line width tends to be the same as the internal velocity structure of diffuse clouds; in contrast, the intrinsic linewidth of $\mathrm{OH}$ is very narrow and it can be easily distinguished in the interior of diffuse clouds. Li \& Goldsmith (2003) have used the combination of narrow-line $\mathrm{H}$ I self-absorption and $\mathrm{OH}$ measurements to probe the atomic to molecular fraction of molecular clouds. Their work has shown that $\mathrm{OH}$ emission is an excellent tracer of the so-called transition clouds, which appear to bridge the gap between cold, neutral clouds and fully molecular clouds. $\mathrm{OH}$ emission has the added advantage that the $\Lambda$ doubling of lines at 1665 and $1667 \mathrm{MHz}$ gives more direct information about the excitation temperature and column density than is available with $\mathrm{H}$ I. Furthermore $\mathrm{OH}$, unlike high frequency molecular gas tracers such as CO, can be observed on the same spatial scales as the atomic gas. Unfortunately $\mathrm{OH}$, like $\mathrm{CO}$, is typically sub-thermal in diffuse clouds and so the emission is very weak. The peak brightness temperatures in Li \& Goldsmith (2003) are only $\sim 0.5 \mathrm{~K}$. 
5.5.1 An OH Survey of the Galactic Plane

To date $\mathrm{HI}$ self-absorption and $\mathrm{OH}$ studies of transition clouds have been pointed observations towards a small number of relatively nearby clouds. We have learned from the H i Galactic Plane Surveys (e.g SGPS; McClure-Griffiths et al. 2005) that H I self-absorption is present along almost all lines of sight in the inner Galaxy. We have also found that the $\mathrm{H}$ i spectral resolution of these surveys is insufficient to fully resolve the linewidth of many of the coldest self-absorption features. The prevalence of self-absorbing clouds in the Galactic Plane suggests that a blind survey of $\mathrm{OH}$ emission would reveal hundreds to thousands of transition clouds at a variety of distances and evolutionary states. This large statistical sample would be invaluable in understanding how molecular clouds form. Combined with information about infrared dark clouds and starless cores, as revealed by MSX and GLIMPSE, we may finally be able to answer: how quickly do molecular clouds form? which clouds form stars? and at what point in a molecular cloud's evolution does star formation begin?

We therefore propose a simultaneous survey of $\mathrm{HI}$ and $1665 \mathrm{MHz}$ and $1667 \mathrm{MHz}$ $\mathrm{OH}$ emission in the Galactic Plane. For each spectral line we require $\sim 5 \mathrm{MHz}$ total bandwidth divided over $\sim 10,000$ channels for spectral resolution of $\sim 0.1 \mathrm{~km} \mathrm{~s}^{-1}$. The survey should cover the area $270^{\circ} \leq l \leq 45^{\circ},|b| \leq 2.5^{\circ}: \sim 700 \mathrm{deg}^{2}$ with an angular resolution of $\sim 2^{\prime}-3^{\prime}$. To detect $\mathrm{OH}$ emission we require a $1 \sigma$ sensitivity limit of $\sim 0.1$ $\mathrm{K}$ in a $0.2 \mathrm{~km} \mathrm{~s}^{-1}$ velocity width. This sensitivity limit will also be more than sufficient to detect $\mathrm{H}$ i self-absorption. 
6 Very Long Baseline Interferometry Science

Lead authors: S. Tingay, N. Bartel

Contributing authors: M. Bietenholz, A. Deller, R. Dodson, P. Edwards, S. Ellingsen, A. Lenc, J. Lovell, C. Phillips, A. Tzioumis

\subsection{Summary}

We describe possible areas of science that can be addressed using VLBI and ASKAP. A number of scientific programs present themselves when considering the use of ASKAP as part of the Australian Long Baseline Array (LBA) and the global VLBI array. Better angular resolution at L-band, better sensitivity, and better $u v$ coverage will aid standard VLBI observations of AGN, pulsars, and $\mathrm{OH}$ masers. An innovative additional capability for ASKAP is multibeaming. If this can be harnessed for VLBI in the form of multiple phased array beams, a number of wide-field survey observations become feasible.

In time, ASKAP should also become part of the recently developed Australian e-VLBI network, currently called PAMHELA. An interesting possibility is to not use ASKAP as part of the LBA, but use it as a source of trigger information for radio transients, as part of ASKAP survey work. These triggers could be transmitted to PAMHELA, and the candidate sources targeted at high angular resolution in rapid follow-up observations. The combination of ASKAP and PAMHELA would be unique and powerful. PAMHELA would add great scientific value to the low resolution detection of transients by ASKAP.

At this point the VLBI community supports the extension of the ASKAP frequency range to S-band $(2.4 \mathrm{GHz})$ but recognises several alternatives to this that may be more cost effective, such as the upgrade of the Ceduna antenna to L-band and/or the use of one of the AuScope geodetic antennas in WA at S-band. All these options should be studied at greater length. The addition of antennas to ASKAP beyond the current plans will make for a more sensitive VLBI array but will not materially open up new areas of science for VLBI.

\subsection{Introduction}

ASKAP consists of an array of 30 antennas of $12 \mathrm{~m}$ diameter, with a maximum baseline of up to $8 \mathrm{~km}$, and a frequency range of perhaps $0.8-2.4 \mathrm{GHz}$. Used as a phased array, ASKAP would have a sensitivity approaching that of Parkes. Good sensitivity, plus its location on the west coast of Australia means that ASKAP can potentially provide substantial gains for Australian and global VLBI in at least the frequency range $1.4-1.7 \mathrm{GHz}$ (L-band).

Within L-band, a number of VLBI science applications are possible, using ASKAP as an additional element in the LBA. Given the phased array field of view (FoV) of approximately $5^{\prime \prime}$, the following observations could be usefully made:

- Active galactic nuclei (AGN) require only a narrow FoV. The addition of long, sensitive east-west baselines is critical for performing high dynamic range imaging of the often complex mas-scale jet radio sources. Further, use of ASKAP along with 
RadioAstron observations will be possible, examining AGN on the longest possible baselines to investigate brightness temperature limits in AGN.

- Pulsar proper motion and parallax observations benefit from good sensitivity on long baselines in order to do the best possible astrometry. The relatively low frequency of ASKAP is optimal for pulsars.

- OH Maser observations benefit from good sensitivity on long baselines, important for resolving individual maser spots.

- The configuration will benefit detailed studies of rare compact objects, such as supernovae seen at both optical and radio wavelengths.

If it is feasible (given the multi-beaming capabilities of ASKAP) to form multiple, independent phased array beams, it will be possible to undertake some forms of widefield imaging, whereby different beams are pointed to different sources, which all lie within the much larger primary beams (compared to the phased-array beam) of the other Australian telescopes. In this way, ASKAP will be capable of undertaking inbeam phase-referenced observations (as are important in pulsar VLBI) to gain extra sensitivity. For observations which are not sensitivity limited but require a large FoV and the $u v$ coverage of ASKAP, the option exists to tie a subset of more closely spaced antennas, sacrificing a small portion of collecting area to increase FoV.

Finally, interesting science is possible with ASKAP if we consider it as a complementary facility to the recently developed Australian e-VLBI capabilities. In particular, some of the most significant work ASKAP will undertake will come from fast, sensitive, high resolution surveys of the entire radio sky. These surveys will uncover radio transient sources in the mJy regime, which ideally should be rapidly followed up at VLBI resolution to examine the details of their structure and evolution. In this case, ASKAP can provide trigger information for real-time e-VLBI observations.

Extending the upper frequency of ASKAP to a frequency of $2.4 \mathrm{GHz}$ (S band) would greatly benefit the Australian VLBI array, because (a) Ceduna (30 m, South Australia) does not have an L-band system and it is required for the best $u v$ coverage, and (b) S-band is a sweet spot in terms of sensitivity across the LBA. For VLBI, extending the ASKAP frequency range to include S-band is strongly encouraged by the Australian VLBI community.

A medium-term alternative could be an L-band upgrade of Ceduna, to achieve the best $u v$ coverage at L-band, rather than to extend the ASKAP frequency range for the best $u v$ coverage at S-band. It is also recognised that the AuScope geodetic VLBI antennas funded through NCRIS 5.13 will operate at S-band and one will be sited in Western Australia. It is possible that this antenna could be used at S-band for LBA observations if available. This antenna would not have the sensitivity of ASKAP, but would provide filling of the $u v$ plane and satisfy a number of science requirements for the applications listed in this document.

We recommend that further work be done to characterise the costs and benefits of a) extending ASKAP to S-band for VLBI; b) upgrading the Ceduna antenna for L-band; c) using the AuScope antenna in WA for astronomical VLBI.

\subsection{ASKAP as a Component of the LBA}

ASKAP will be an additional antenna in the LBA and will also be capable of participating in global VLBI experiments, at frequencies of 1.4 and $1.6 \mathrm{GHz}$. Using a single 
phased array beam from ASKAP with the existing Australian LBA will allow many VLBI experiments to be performed, those that do not require an FoV larger than a few arcseconds in diameter (due to the phased array FoV limitations of maximum baseline lengths of $8 \mathrm{~km}$ ). These experiments include observations of AGN and of pulsars.

\subsubsection{UV Coverage}

Figure 33 shows the improvement in the $u v$ coverage by including ASKAP in the LBA at $1.6 \mathrm{GHz}$. The maximum baseline is greatly increased and east-west baselines are formed to complement the north-south baselines of the east coast antennas.
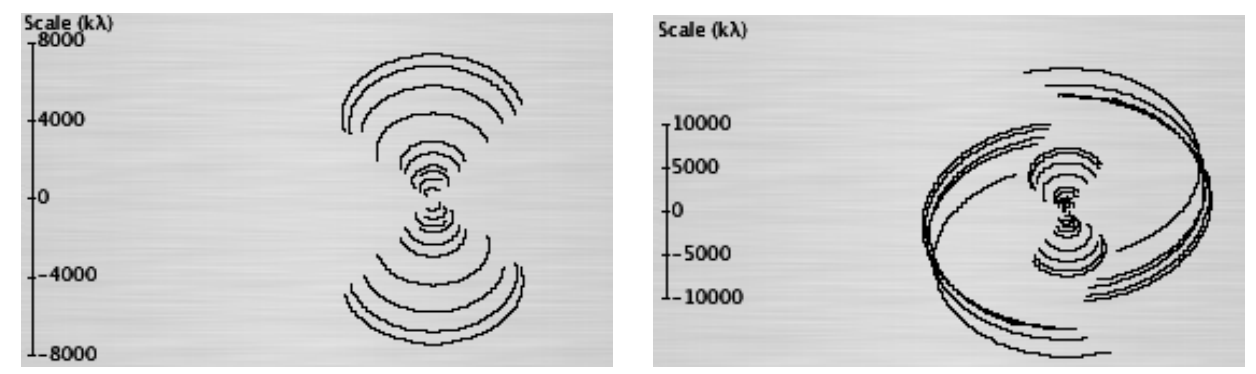

Fig. 33 LEFT: $u v$ coverage of the LBA at a frequency of $1.6 \mathrm{GHz}$ (ATCA, Parkes, Mopra, Hobart, Tidbinbilla) for a source at $\delta-45$ degrees observed for 12 hours. RIGHT: The $u v$ coverage from an identical observation but including ASKAP in the LBA.
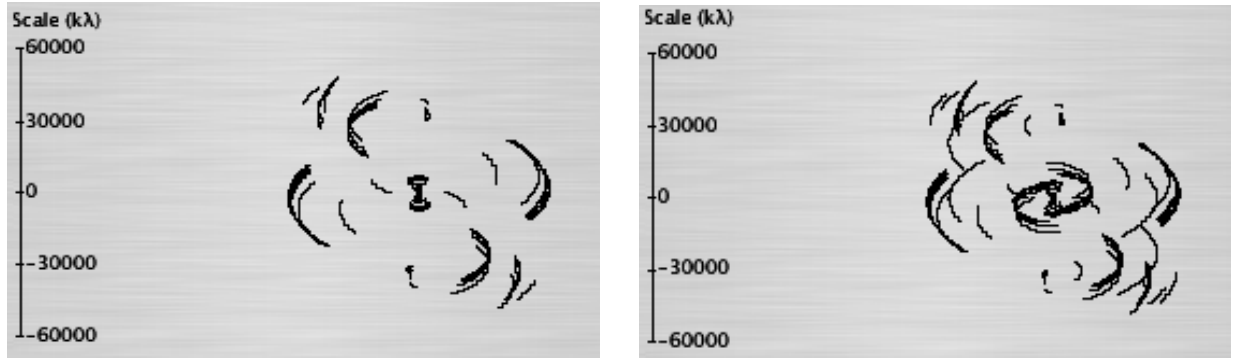

Fig. 34 LEFT: $u v$ coverage of a global VLBI array at a frequency of $1.6 \mathrm{GHz}$ (ATCA, Parkes, Mopra, Hobart, Tidbinbilla, Kashima, Hartebeesthoek, VLBA_OV, VLBA_MK) for a source at $\delta-30$ degrees observed for 12 hours. RIGHT: The $u v$ coverage from an identical observation but including ASKAP in the global array.

Significant gains are also apparent for global VLBI observations that use ASKAP. Figure 34 shows the improvement in $u v$ coverage by the addition of ASKAP into an array of antennas in Australia, Japan, South Africa, and the USA. ASKAP significantly closes a number of holes in the $u v$ coverage. 
For the observational cases shown above in Figure 33, the sensitivity ( $1 \sigma$ image rms) of the LBA alone is $25 \mu \mathrm{Jy}$ /beam (assuming a bandwidth of $128 \mathrm{MHz}$, dual polarisation at ATCA, Parkes, Mopra and $64 \mathrm{MHz}$, dual polarisation at Hobart and Tidbinbilla). Along with three times the angular resolution, the inclusion of ASKAP (at $128 \mathrm{MHz}$ dual polarisation) improves the sensitivity of the array to $18 \mu \mathrm{Jy} / \mathrm{beam}$.

For the observational cases shown in Figure 34, the sensitivity of the global VLBI array is $22 \mu \mathrm{Jy} /$ beam. Although the inclusion of ASKAP does not add longer baselines to the global array, it significantly improves the $u v$ coverage, particularly on intermediate baselines and improves the sensitivity to $17 \mu \mathrm{Jy} /$ beam.

In general, the improved $u v$ coverage obtained by including ASKAP in both Australian regional and global VLBI arrays will reduce de-convolution errors in imaging that currently limit the achievable sensitivity to a factor of a few above the theoretical sensitivities given above.

\subsubsection{Imaging over Wide Fields}

Adding ASKAP to the LBA offers a number of interesting opportunities for wide-field VLBI science. The full potential of such an array will depend greatly on the configuration of ASKAP itself, since the overall FoV will be almost completely constrained by its synthesised primary beam. At L-Band this equates to an FoV of $\sim 6^{\prime \prime}$ FWHM with the entire $8 \mathrm{~km}$ array. While this is not useful for large-scale survey work, the combined increase in sensitivity and resolution of this array could be used to survey and monitor SNRs in starburst regions out to $20 \mathrm{Mpc}$ with sub-parsec spatial resolution. Such surveys can be used to determine limits on the star-formation rate and supernova rate in the local Universe (Lenc \& Tingay 2006).

For more general VLBI surveys a greater FoV to increase the survey speed would be beneficial. Since the available FoV is inversely proportional to the maximum baseline length, an increase in FoV can be achieved by utilising a smaller subset of ASKAP. For example, if a $1 \mathrm{~km}$ diameter core of ASKAP is utilised as part of the LBA then the available FoV can approach $\sim 1^{\prime}$ FWHM. This of course comes at the cost of reduced sensitivity since only a fraction of ASKAP would be utilised. To minimise the impact on the sensitivity, the array could be designed with a denser population of antennas in the inner $1 \mathrm{~km}$ core. Such an array could be used to survey and study the morphology and population of galaxies in the early Universe (Jackson 2004). Combined with observations at other wavelengths, this would improve our understanding of the evolution of galaxies in the early Universe.

The Focal Plane Array (FPA) receivers on ASKAP allow a further method to increase FoV. The currently proposed 200 element FPAs have the potential to form up to 50 independent phased array beams, increasing the observable FoV by a factor of 50 in angular area and thus increasing the survey speed by the same factor. When combined with the $1 \mathrm{~km}$ subset array an FoV as wide as $\sim 7^{\prime}$ (comparable to the FoV of the Tidbinbilla $70 \mathrm{~m}$ antenna at L-band) may be achieved, increasing the overall survey speed by a factor of 350 . Operating ASKAP in this way will introduce some technical challenges for VLBI. 


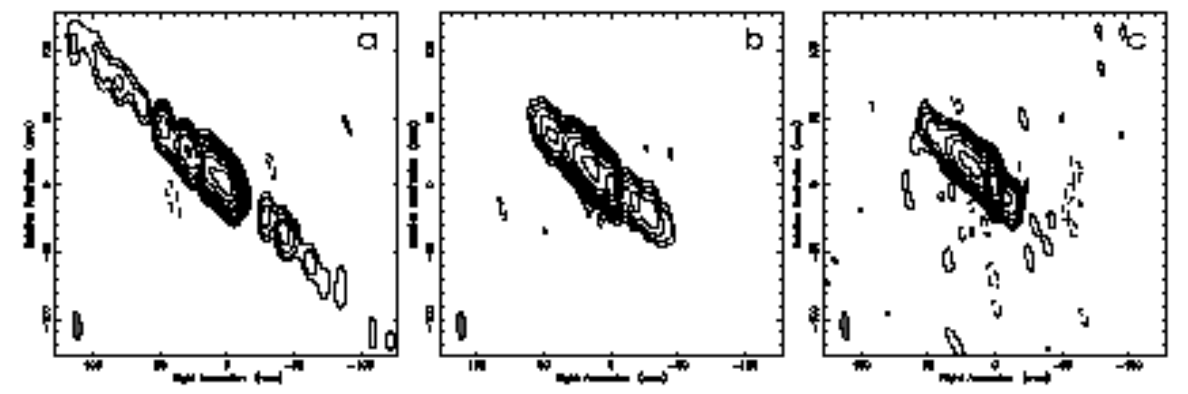

Fig. 35 Images at 2.3, 4.8 and $8.6 \mathrm{GHz}$ (left to right, respectively) of the AGN jet in Centaurus A, showing the frequency-dependent structure on mas scales (Tingay \& Murphy 2001).

\subsubsection{AGN, Space VLBI, and GLAST}

The nuclei of AGN are structurally, kinematically, and spectrally complex, involving relativistic jets of material on parsec (mas) scales that evolve with time and frequency (e.g. as seen in AGN such as that of Centaurus A; see Figure 35). VLBI is the only direct method with which AGN and these jets can be probed on mas scales. Of prime importance for AGN VLBI are length of baseline, sensitivity of array, and uniformity of $u v$ coverage. The addition of ASKAP to the LBA and global VLBI array makes substantial improvements in these regards, as seen in earlier sections.

As well as for complicated radio AGN structures, the inclusion of ASKAP will add extra sensitivity for the detection and study of weak AGN, at the mJy level. Recent results from the LBA show the value of VLBI follow-up of large-area radio surveys such as AT Large Area Survey (ATLAS: Norris et al. 2007). A detection of one of these ATLAS sources indicates a radio bright, but infrared faint object. The study of more sources in these radio deep fields with VLBI allows investigation of the importance of star formation vs AGN activity in the early formation of galaxies.

The Russian space VLBI mission RadioAstron is making progress toward launch in (possibly) 2009 and will operate at, among other wavelengths, 92 and $18 \mathrm{~cm}$, which are within the ASKAP frequency bands. The use of Parkes/Tidbinbilla on the east coast and ASKAP on the west coast will allow sensitive coverage for southern hemisphere RadioAstron observations. The long baselines provided by space VLBI will explore the high brightness temperature limits of AGN. This work carries on from the results obtained from the LBA in conjunction with the VSOP space VLBI mission (Horiuchi et al. 2004; Tingay et al. 2002) which found a large fraction of unresolved nuclei in bright AGN, and intra-day variability work which has found evidence for structures of $10 \mu$ as in $56 \%$ of flat-spectrum AGN (Lovell et al. 2003, 2007). RadioAstron will be capable of directly resolving these sources for the first time.

Finally, the next generation gamma-ray telescope, the Gamma-ray Large Area Space Telescope (GLAST), will be flying when ASKAP starts operation. There will be a significant collaborative effort between the GLAST mission and ground-based VLBI arrays in monitoring GLAST AGN targets. As the southern hemisphere VLBI array, the LBA would benefit from the use of ASKAP for monitoring gamma-ray AGN, both in the detection of weak sources and the study of their high resolution structures. The LBA has been used to conduct previous work in the southern hemisphere (Tingay et 
al. 1996) targeting the bright gamma-ray AGN discovered by the EGRET instrument aboard the Compton Gamma-Ray Observatory (CGRO).

AGN observations would greatly benefit from the higher angular resolution and sensitivity possible if ASKAP had an S-band capability.

\subsubsection{Pulsar Parallaxes and Proper Motions}

VLBI astrometry of pulsars is crucial for obtaining model-independent distances and transverse velocities, and thus calibrating the pulsar distance scale, luminosity function and Galactic electron distribution (see e.g. Brisken et al. 2002). However, pulsars are difficult targets for VLBI. Many are comparatively weak, requiring sensitive VLBI arrays, and their steep spectral indices force observations to be undertaken at low frequencies, typically L-band. Observing at low frequencies both decreases angular resolution, requiring higher signal-to-noise detections and better control of systematic errors to obtain a given positional accuracy, while simultaneously increasing systematic uncertainties due to larger phase errors from rapidly changing ionospheric gradients.

The LBA is the only existing southern VLBI array that is sensitive enough to undertake astrometry studies of faint pulsars. However, the existing LBA is limited in its capabilities for L-band astrometry. This is primarily because the Ceduna $30 \mathrm{~m}$ telescope in South Australia does not possess an L-band receiver, due to its beam waveguide construction. Without Ceduna, the LBA is reduced to a roughly northsouth arrangement of telescopes, with a detrimental effect on $u v$ coverage. The Ceduna baselines are the longest in the LBA, and their removal roughly doubles the extent of the synthesised beam in the east-west direction. Finally, the reduction to 5 antennas (or 4 when, as is common, the NASA DSN antenna at Tidbinbilla is unavailable) reduces the number of available baselines from 15 to 10 (or 6), making control of systematic uncertainties more difficult.

The addition of the phased ASKAP to the LBA essentially offsets the loss of Ceduna at L-band, but with two additional benefits:

1. Being located approximately $1600 \mathrm{~km}$ further west than Ceduna, ASKAP offers a doubling of resolution in one axis (i.e. four times the existing L-band resolution); and

2. ASKAP is much more sensitive than Ceduna, being a $64 \mathrm{~m}$ equivalent compared to a $30 \mathrm{~m}$. Moreover, this sensitivity contributes to the longest baselines whereas currently with the LBA at L-band the longest baselines are to the less sensitive $26 \mathrm{~m}$ dish at Hobart. When uniform weighting is used (which is typically the case for astrometry), the improvement in image sensitivity is striking: $43 \mu \mathrm{Jy}$ with ASKAP compared to $98 \mu \mathrm{Jy}$ without, while if Tidbinbilla is unavailable $56 \mu \mathrm{Jy}$ can still be obtained, compared to $140 \mu \mathrm{Jy}$ without ASKAP. These calculations assume a 10 hour phase referenced observation with a $50 \%$ calibrator/target duty cycle.

The combination of these benefits would allow the LBA to target a much wider range of pulsars for astrometry than is presently feasible. Pulsars, kicked by their supernova explosions, have high and easily detected proper motions. These have been found to lie along the spin axes (Johnston et al. 2005), and therefore can be used to provide insights into the initial blast of the supernova and the pulsar characteristics.

VLBI of pulsars also has benefits for tying together astronomical reference frames. The determination of the positions of pulsars in the earth-spin oriented extragalactic 
reference frame combined with position determinations from pulse timing in the earthorbit oriented reference frame provides a tie of the two reference frames. This tie is presently established at the milliarcsecond level through a couple of millisecond-pulsarQSO pairs in the Northern Hemisphere (Nunes \& Bartel 1998). Additional observations of millisecond-pulsar - QSO pairs in the Southern Hemisphere would help to provide a uniform tie of the two reference frames. This tie could be used for, e.g., impoved spacecraft navigation. It could also be used for a gravitational redshift test of the earth in the solar potential.

An S-band capability for ASKAP would be very useful for proper motion, parallax and astrometry observations, giving high sensitivity on the longest baselines.

\subsubsection{OH Masers}

The ground-state lines for the hydroxyl radical $(\mathrm{OH})$ occur at a frequency of about $1650 \mathrm{MHz}$. $\mathrm{OH}$ is a common molecule and has been observed to undergo maser action in a variety of astrophysical environments. Maser emission from the main lines at 1665 and $1667 \mathrm{MHz}$ are commonly observed towards high-mass star formation regions, while emission from the satellite line at $1612 \mathrm{MHz}$ is common towards late-type stars and $1720 \mathrm{MHz}$ masers are observed in regions where SNRs are interacting with molecular gas.

High resolution observations from long baseline arrays show highly complex and varied structures for the various maser species associated with high-mass star formation regions, with spherical expansion, bipolar outflows, disks and rings, bow-shocks and infall. The different maser species typically trace different parts of the star forming regions. Combining high-resolution $\mathrm{OH}$ imaging with observations of methanol and water masers, and the high velocity resolution dynamical information, it is possible to image and interpret the three-dimensional distribution of masers in great detail. In some cases it is possible to obtain proper motions and accurate distance estimates. The inclusion of ASKAP as an element of the LBA will significantly increase the resolution of $\mathrm{OH}$ maser observations and make them comparable to that achievable for the higherfrequency methanol and water maser observations using only the existing LBA.

Magnetic fields play a critical role in star birth and star death. $\mathrm{OH}$ masers provide an excellent means of studying the magnetic fields in the regions where they are located, as strong polarization is observed from the Zeeman effect, with line-splitting often significantly larger than the line width. Magnetic fields of several $\mathrm{mG}$ are typically measured in both star-formation regions and stellar winds. Multiple spectral components and variations in the magnetic field strength within a region mean that VLBI observations are required in order to unambiguously identify Zeeman pairs and hence to obtain accurate magnetic field estimates.

$\mathrm{OH}$ masers (strongest in the $1667 \mathrm{MHz}$ transition) have also been detected towards late-stage mergers which are ultra-luminous infrared galaxies (ULIRGs). Much of the maser emission in these sources is diffuse and resolved out on long baselines. However, compact emission is observed where the masing gas amplifies compact background sources such as supernovae in the host galaxies. Through observing the changes in the compact emission over time it is possible to investigate the supernova rate in these starburst regions, which cannot be done through other means. 
There are types of celestial compact objects sufficiently bright for VLBI observations with ASKAP that are only sparsely distributed over the sky. With additional VLBI observations in the southern hemisphere a precious few more could be observed. These sources are supernovae, radio stars, microquasars, and X-ray sources. As an example we show an image of supernova 1993J in Figure 36.

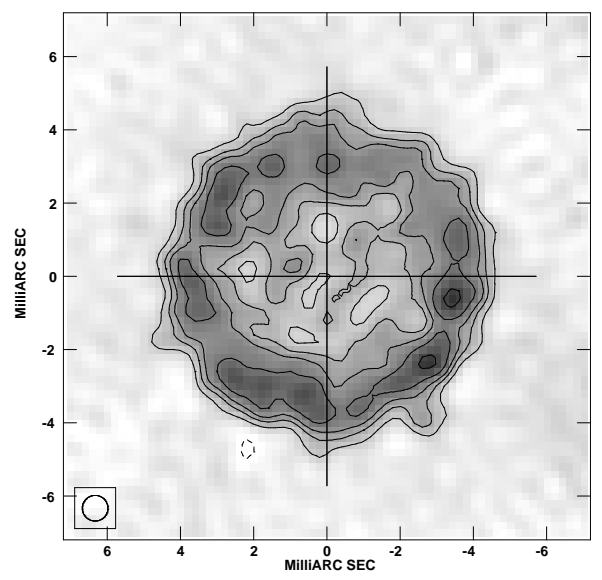

Fig. 36 An composite image of SN 1993J made from 8.4 GHz maps from Dec. 1998 to Nov. 2000. Contours are at $-16,16,32,45,64$, and $90 \%$ of the peak brighness. The background rms is at $5.3 \%$ of the peak brightness (Bietenholz, Bartel, \& Rupen 2003).

Monitoring a supernova with VLBI gives a set of observables essential for modelling the interaction between the ejecta and the circumstellar medium. It can help in determining the density profile of the latter and provide clues as to the history of the mass-loss of the progenitor tens of thousands of years before it exploded. It may also give evidence for a neutron star or a black hole lurking in the center of the supernova as the stellar corpse from the explosion. Further, a geometric distance to the supernova and its host galaxy may be determined, using the expanding shock front method by combining in a model the transverse expansion velocity determined from VLBI and the radial expansion velocity determined from the Doppler broadening of optical lines (Bartel et al. 2007).

Only three supernovae related to optical events have been imaged with VLBI in detail so far, all of them located in the northern hemisphere. Only one supernova located in the southern hemisphere was imaged, with the ATCA, namely SN 1987A. ASKAP VLBI will increase the number of supernovae that can be imaged and contribute to our understanding of the diversity of supernova morphologies and their evolutions to supernova remnants.

In general, ASKAP as a poweful node in a southern VLBI array will significantly improve our knowledge of relatively rare compact objects. 

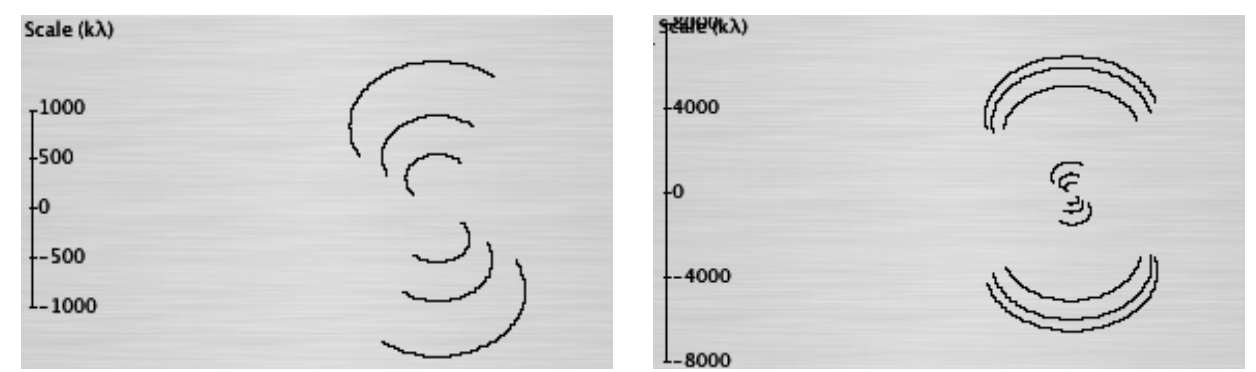

Fig. 37 LEFT: $u v$ coverage of PAMHELA at a frequency of $1.6 \mathrm{GHz}$ (ATCA, Parkes, Mopra only) for a source at $\delta-45$ degrees observed for 12 hours. RIGHT: Full PAMHELA (ATCA, Parkes, Mopra, Hobart).

\subsection{ASKAP and PAMHELA for Rapid Transient Science}

A significant part of the ASKAP science case involves the rapid survey capability of the instrument and its ability to detect rapid transients in continuum emission. The types of transient that ASKAP will likely detect include galactic X-ray binaries, gamma-ray bursts (GRBs), flaring stellar systems, and possibly supernovae in nearby galaxies. The event rate and detection limits required for each of these transient types varies significantly. Chapter 8 of this document deals with the details of transient surveys with ASKAP.

In the case of the known and yet to be discovered transients, it is likely that the objects in question will be compact and will have structures that evolve rapidly. This is certainly true of the known transient sources such as X-ray binaries, GRBs, and supernovae. Following detection with ASKAP, very useful observations could be made with a VLBI array. For example, is compact structure present in the transient source? What are the morphologies of the compact structures and how do they evolve with time? What are the multi-frequency characteristics of the transient sources above 1.7 $\mathrm{GHz}$ ? These questions will allow the underlying source of the radio emission (supernova, black hole binary, GRB) to be investigated, determining the emission mechanism at radio wavelengths in combination with possible multi-wavelength observations from high energy and optical observatories.

We propose using ASKAP in stand-alone mode for transient detection. We propose that transients that satisfy certain criteria trigger rapid follow-up observations with PAMHELA, the Parkes, ATCA, Mopra, Hobart Electronic Long-baseline Array. Example $u v$ coverages for PAMHELA are shown in Figure 37.

\subsubsection{Transient Detection with ASKAP and Trigger Criteria for PAMHELA}

Given uncertainties regarding the transient detection rate from surveys with ASKAP (see Chapter 8), the definition of trigger criteria at this stage is premature. However, some approximate guidelines can be derived from the capabilities of ASKAP, the capabilities of the VLBI array, and practical considerations of dynamically scheduled VLBI observations.

Since transients will occur at random times and follow-up VLBI observations would potentially disrupt observers on the Parkes, ATCA, Mopra, and Hobart telescopes, we should adopt strategies that limit the disruption to ATNF users. Given the $1 \sigma$ 
sensitivity of ASKAP in a 60 second pointing of $0.3 \mathrm{mJy}$ and assuming $5 \sigma$ is required for a transient detection (1.5 mJy), an observation with Parkes, the phased ATCA, and Mopra would require an integration time of approximately 160 seconds to detect such a source at the same significance (assuming it to be unresolved) on the ATCA-Parkes baseline.

We envisage a system in which transient detections greater than 2 mJy trigger a PAMHELA observation of approximately 15 minutes, under certain circumstances. The trigger criteria for PAMHELA observation could therefore look something like:

- Is transient detection by ASKAP > 2 mJy at $1.6 \mathrm{GHz}$ ?

- Is the transient at an hour angle that can be observed by PAMHELA for $>1 \mathrm{hr}$ ?

- Is a suitable phase reference source nearby on the sky?

- Is a 1.6 GHz observation currently possible at all antennas? Is PAMHELA follow-up possible at another frequency?

- Are scientific programs at the PAMHELA antennas highly ranked enough that disruption should not occur?

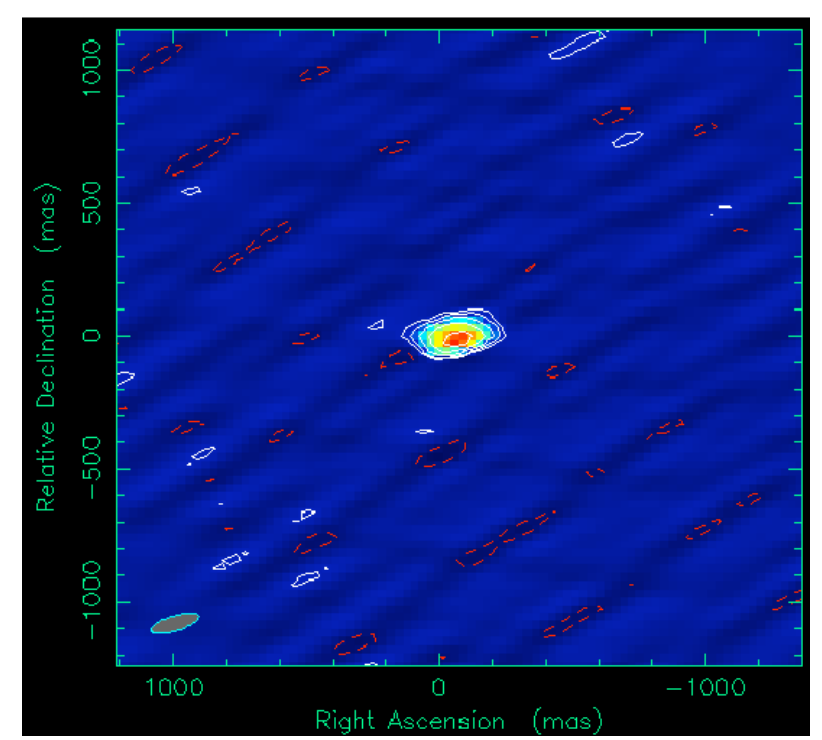

Fig. 38 An e-VLBI image of Circinus X-1 from March 2007, realised with PAMHELA running at 256 Mbps (Phillips et al. 2007). The total flux of the compact component is approximately $10 \mathrm{mJy}$. Observations were approximately $3 \mathrm{hrs}$ in duration over 2 nights.

\subsection{2 e-VLBI Capabilities and Example Science}

The capabilities for e-VLBI in the form of PAMHELA are currently under development as a collaboration between ATNF and the Swinburne University of Technology, who provide a software correlator (Deller et al. 2007a). Recently (March 2007) the first PAMHELA observation of the X-ray transient Circinus X-1 was conducted at a data rate of $256 \mathrm{Mbps}$ on Parkes, the ATCA, and Mopra and at $128 \mathrm{Mbps}$ on Hobart (Phillips 
et al. 2007; Deller et al. 2007b; see Figure 38). These successful PAMHELA tests lay the basis for rapid VLBI follow-up of ASKAP transient detections. PAMHELA as an e-VLBI instrument is demonstrating technologies relevant to the SKA (long-haul data transmission via fibre and real-time correlation); it is therefore important to include ASKAP as an extension to PAMHELA as soon as is practically possible.

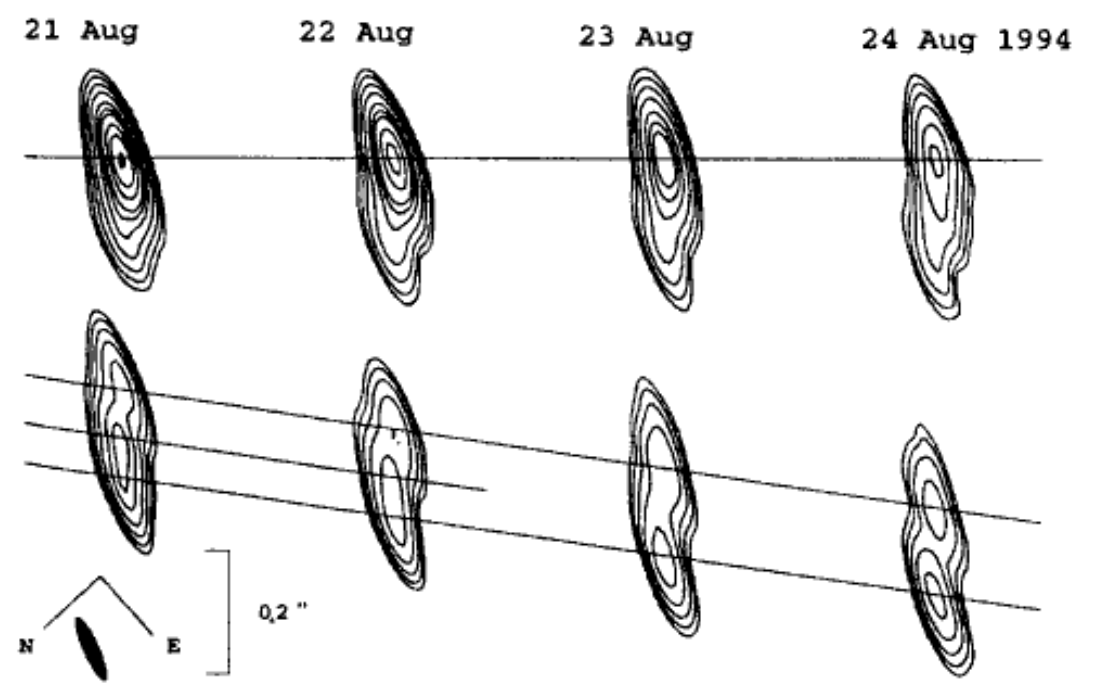

Fig. 39 Discovered with MOST, using a trigger instrument analog to ASKAP, GRO J1655-40 was observed with rapid turnaround using the Parkes - Tidbinbilla interferometer (PTI) to determine compact structure. This montage was made from four LBA observations planned and executed based on the rapid PTI results (Tingay et al. 1995).

X-ray binary systems such as Circinus X-1 are a major target for PAMHELA observations triggered by ASKAP. The X-ray binary GRO J1655-40 was studied 10 years ago using a similar system (see Figure 39). GRO J1655-40 was an X-ray binary with multi-Jy radio emission which revealed apparent superluminal motions in the compact jet components (Tingay et al. 1995). The goal of the transient studies would be to investigate the properties of the X-ray transient population at the mJy level, up to two orders of magnitude fainter in radio emission.

\subsubsection{Complications}

The dynamic scheduling of PAMHELA to respond to ASKAP transient detections would likely require a significant software effort to implement, and a high level of coordination between the ATNF observatories. The advantage of such a system is that every interesting transient can potentially be followed up with PAMHELA observations.

It may turn out that such a system is impractical, however. A logistically simpler but scientifically perhaps less useful approach would be to schedule dedicated PAMHELA time periodically during ASKAP transient surveys. In this way, perhaps 12 hours per week could be scheduled for PAMHELA to observe all ASKAP transients detected over the period of the previous week. 
7 Pulsar Science

Lead authors: G. Hobbs, V. Kaspi

Contributing authors: M. Bailes, S. Chatterjee, S. Johnston, R. Manchester, I. Stairs

\subsection{Summary}

Since the initial discovery of pulsars in Cambridge (Hewish et al. 1968), radio telescopes world-wide have played a major part in the discovery of new pulsars. Pulsars have been used as tools to address some of the most fundamental questions in basic physics, allowing precision tests of general relativity, investigations into the equation of state of ultradense matter and the behaviour of matter and radiation in the highest magnetic fields known in the universe. The commissioning of ASKAP will provide the transition between the use of large single-dish instruments (such as the Parkes and Arecibo telescopes) to using large numbers of small antennas with a wide field-of-view $(\mathrm{FoV})$ as is likely in the final mid to high frequency SKA design.

Applications of pulsar observations include a very wide variety of interesting astrophysical topics, often with unparallelled precision. Such topics include binary evolution, binary dynamics, the interstellar medium, globular cluster physics, supernova remnant astrophysics, the physics of relativistic winds and precision astrometry. Pulsars are also instrinsically interesting, being the result of core collapse supernovae and astonishing converters of mechanical energy of rotation into electromagnetic radiation, particles and magnetic fields. The study of pulsars themselves is important for constraining the overall population's properties and hence their origin, as well as understanding the mysterious pulsar emission mechanism.

Historically it has been common to carry out pulsar research using large single dish instruments. ASKAP provides a pathway between the current systems and the full-scale SKA. ASKAP will be ideal for carrying out relatively fast all-sky surveys that will allow $\sim 1000$ pulsars to be discovered. In this chapter, we show that it will be possible for ASKAP to continue to observe these (and previously known) pulsars in order to determine their astrometric, spin and orbital parameters. ASKAP will, for several millisecond pulsars, be able to provide data suitable for integration into a global timing array project which aims to detect low-frequency gravitational wave sources.

Pulsar observations, especially searches, with ASKAP will present several logistical and computational challenges, some of which can be mitigated through specification choices. In particular, any large-scale survey with good sensitivity will be severely limited computationally; the need to process every pixel independently ensures that this will become worse as the square of the maximum baseline length. It is therefore necessary that as many short baselines as possible be present in the configuration of ASKAP. For pulsar timing, there is no such requirement; any configuration is adequate.

The expansion of ASKAP would be good for several reasons. Most important is the increase in instantaneous sensitivity through both the addition of more telescope collecting area and a reduction in system temperature. These will ensure that ASKAP will become more competitive in searching and in high precision pulsar timing, leading to improved data-sets for inclusion in global timing array projects. More array elements will also ensure that more short baselines are present in the array. 


\subsubsection{Searching for Pulsars}

The current pulsar catalogue lists more than 1750 known pulsars (Manchester et al. 2005). Approximately 880 of these were discovered using the Parkes $20 \mathrm{~cm}$ multibeam receiver (e.g. Manchester et al. 2001). Pulsar surveys need to take into account that 1) a typical pulsar only radiates strongly for a small percentage of its period, 2) postdetection filtering and the propagation of the signal through the interstellar medium broaden the observed pulse widths, 3) the pulsar signal is also dispersed as a function of frequency due to the interstellar medium, 4) pulsars have a wide range of periods from $\sim 1 \mathrm{~ms}$ to $10 \mathrm{~s}$, and 5 ) pulsars are usually intrinsically weak radio sources and have steep spectral indices. We highlight the different types of known pulsars and their range in pulse periods and spin-down rates in Figure 40. With roughly 120,000 potentially observable radio pulsars in the Galaxy (Faucher-Giguere \& Kaspi 2006), continued searching is important not only for fleshing out the true properties of the pulsar population, but also because within that group there are likely to be many interesting "jewels". One such "jewel" would be the discovery of a millisecond pulsar/black hole binary for testing general relativity and even the Cosmic Censureship Conjecture and No-Hair theorem (Kramer et al. 2004).

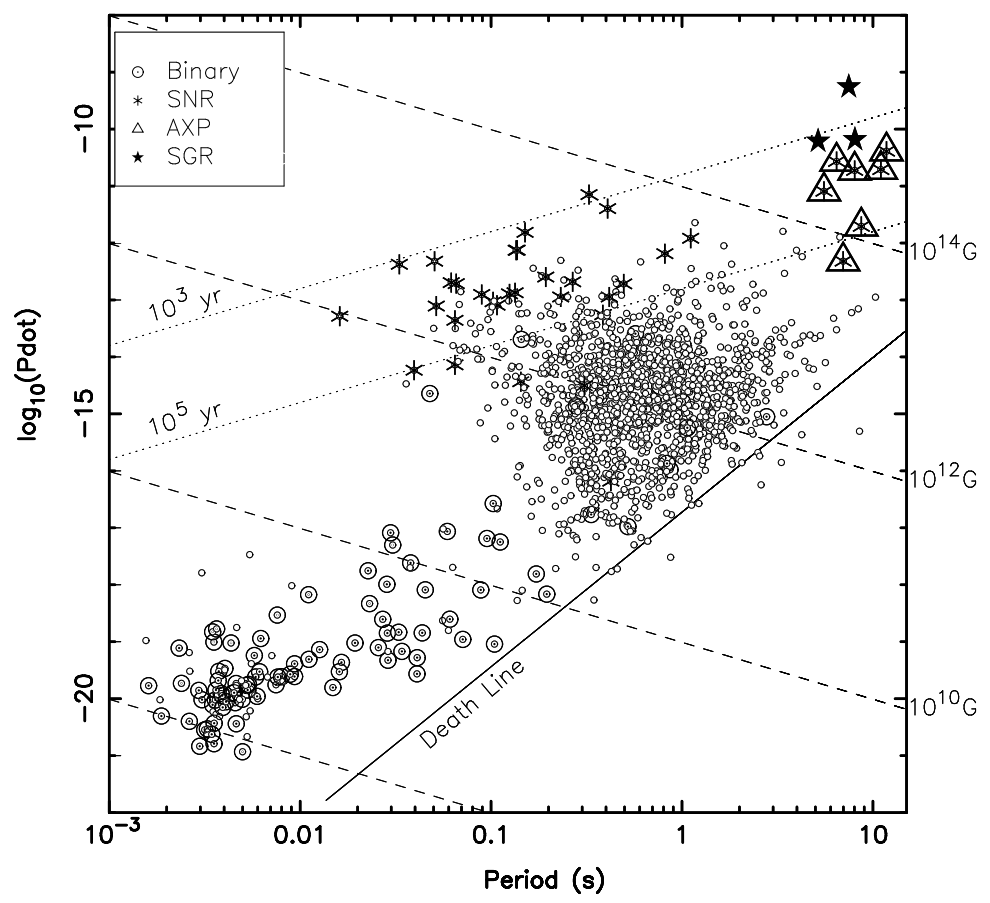

Fig. 40 The Period-Period-derivative plane for the currently known pulsars and magnetars (AXPs and SGRs) in the field of the Galaxy. Lines of constant magnetic field and characteristic age, both estimated under the magnetic dipole assumption, are indicated. Plausible associations with supernova remnants (SNRs) are shown by small stars, and binary pulsars are identified as circles with central dots. 
Pulsar surveys are designed to maximise the number of new pulsars discovered; this leads to a choice between observing frequency, back-end systems and sampling time within the instrumental constraints. The observing frequency of $\sim 1 \mathrm{GHz}$, the on- and off-line processing power, and the large FoV make ASKAP a powerful instrument for carrying out a new pulsar survey. It is essential to continue undertaking pulsar surveys in order to detect the iceberg of sources that must exist in the Galaxy, only the tip of which can we see today. Pulsars may not have been found in previous surveys due to interference in the data, the pulsar being "switched off" or eclipsed for long time periods, or the neutron star precessing and moving the radio beam into and out of our line of sight. The science case for detecting new pulsars is wide-ranging and includes:

- The ability to improve models of the pulsar population and hence determine birth rates and the Galactic distribution (e.g. Vranesevic et al. 2004).

- The discovery of unique objects, particularly highly relativistic binary systems including pulsar - black hole binary systems. Such systems are unique testbeds for General Relativity and alternate theories of gravity.

- The discovery of pulsars useful for specific topics of research. These include young pulsars for testing QED and constraining the dense matter equation of state; and highly-stable millisecond pulsars for inclusion in pulsar timing array projects. (A pulsar timing array is useful for detecting gravitational waves and constraining cosmological models of the early Universe; see Section 7.2.2.)

- Mapping the electron density distribution of the Galaxy. Any pulsar survey provides an estimate of a pulsar's dispersion measure. Combining this measurement with an estimate for the pulsar's distance (obtainable using timing, VLBI or H I absorption studies) allows the mean electron density in the direction of the pulsar to be determined (e.g. Cordes \& Lazio 2002).

- Determining the Galactic magnetic field. Combining measurements of pulsar rotation measures and dispersion measures provides a measurement of the magnetic field strength along the line of sight to the pulsar. Combining these values with estimates of the pulsar distances allows the Galactic magnetic field to be mapped (e.g. Han et al. 2006).

- Understanding the pulse emission mechanism. As the number of known pulsars increases so does the variety in pulse profiles, polarisation and fluctuation properties. A more complete understanding of the pulse emission mechanism will only occur from the careful analysis of these properties for a large sample of pulsars at multiple observing frequencies (e.g. Karastergiou \& Johnston 2006).

ASKAP will be able to perform sensitive all sky pulsar surveys. A straight-forward survey described below would be 10 times more sensitive than previous all-sky pulsar surveys carried out in the Southern hemisphere and could detect up to 1600 new pulsars.

\subsubsection{An Example All-Sky Survey}

The unique capability of ASKAP that enhances pulsar surveys is the simultaneous availability of multiple beams covering a large FoV allowing the rapid survey of large areas of the sky. The survey speed of the telescope, given in equation 2, suggests that ASKAP will be $13 \times$ faster than the Parkes Multi-beam Pulsar Survey that detected 880 new pulsars in a $10^{\circ}$-strip of the Galactic plane (Manchester et al. 2001) and $\sim 350 \times$ faster than the previous Parkes all-sky survey (Lyne et al. 1998) carried out 
at $436 \mathrm{MHz}$. However, the caveat here is that we need to able to record and process (coherently) the gigantic data sets such a survey produces. We return to this below.

We suggest a survey with ASKAP which occupies 100 days of observing time and covers the 30,000 square degrees of visible sky. Each $30 \mathrm{deg}^{2}$ single pointing therefore is observed for $\sim 150$ min. Such a survey, with the strawman ASKAP parameters, would equal the sensitivity of the Parkes Multi-beam Pulsar Survey and would be 10 times more sensitive than the previous Parkes all-sky survey. Data recording requirements (see below) would force the survey to sample at a rate of only 5 or $10 \mathrm{~ms}$; this would necessarily preclude sensitivity to millisecond pulsars (MSPs) with current disk technology.

Simulations (Lorimer; private communication) show that such a survey would detect 1600 pulsars with periods greater than $\sim 40 \mathrm{~ms}$, about half of which would be new discoveries. Many of the new detections would be low-luminosity objects; a large sample of low-luminosity pulsars is important in defining pulsar birthrates and evolution. Furthermore, even though this proposed survey would not be sensitive to millisecond pulsars, many exotic objects have longer periods including the original binary pulsar PSR B1913+16 (Hulse \& Taylor 1975), pulsars with high-mass companions, and relativistic systems such as PSR J1141-6545 (Kaspi et al. 2000). It is likely that any pulsar companion to a black hole would be slow spinning and the survey described here might find such an object. Finally this survey would be sensitive to transients with pulse widths greater than $10 \mathrm{~ms}$ or so.

\subsubsection{Requirements}

The computing requirements for the pulsar survey are very large. The rate at which the data must be collected is given by

$$
\mathrm{DR}=\tau \times c \times b \times M \times B
$$

where $\tau$ is the sampling rate, $c$ is the number of channels across the band, $b$ is the number of bits, $M$ is the number of fields-of-view and $B$ the number of baselines. For the ASKAP parameters, a survey for millisecond pulsars with $b=8, c=512$, $\tau=10000 \mathrm{~Hz}$ gives a data rate of $\sim 65$ Gbytes/sec, much too large by a factor of at least 100. Therefore, we are forced to abandon a search for MSPs and rather settle for parameters like $b=2, c=64, \tau=200 \mathrm{~Hz}$ which yield a data rate of $\sim 40 \mathrm{Mbytes} / \mathrm{sec}$ and a single pointing total of $\sim 450$ Gbytes. This sort of data rate implies that the data can be sent down the optical fibre to the processing centre in Geraldton/Perth.

Data processing involves first forming a time series for a given pixel within each primary beam (computationally cheap) and then using standard pulsar search techniques. The processing power need scales as

$$
\mathrm{PP} \propto L^{2} D^{-2} M
$$

where $L$ is the maximum baseline length and $D$ the dish diameter. This equation implies that minimising $L$ is vital for data processing. For the survey described above, if $L$ is of order $\sim 1 \mathrm{~km}$ the survey processing (assuming a 100 node cluster) will take a factor 10x more than the observing time.

It is therefore clear that the ASKAP design is far less efficient for large-area pulsar surveys than is a single aperture instrument. Optimising for pulsar science clearly demands a large total aperture and as much central concentration of the aperture as possible, in addition to a low system noise. 
7.1.3 Targeted Pulsar Searches: Supernova Remnants, Globular Clusters and the Magellanic Clouds

An ASKAP phased-array search for young pulsars having periods $>10 \mathrm{~ms}$ in supernova remnants is feasible and could offer comparable sensitivity to a similar search at the Green Bank Telescope, albeit with much greater computational requirements. The wide primary beam of ASKAP can be seen as a plus in this case, allowing sensitivity to pulsars that have moved away from the centre of the remnant. The vast majority of catalogued SNRs have no known associated pulsar (although some appear to contain other types of neutron stars such as magnetars or central compact objects). Thus an ASKAP survey of southern, thus far empty, SNRs would be of interest. The discovery of pulsars in supernova remnants is of great scientific value. For most of these pulsars it is possible to obtain independent age and distance estimates. These pulsars are generally unstable rotators which allow their interior structure to be probed by studying their spin-down. Typical pulsar birth velocities of $\sim 400 \mathrm{~km} \mathrm{~s}^{-1}$ are much faster than their progenitor stars (Hobbs et al. 2005). Analysing the velocity and polarisation properties of young pulsars allows the proposed mechanisms for producing such high velocities to be studied (Johnston et al. 2005).

Globular clusters are also very interesting targets for pulsar searches. Recent observations of globular clusters have led to the discovery of the fastest spinning pulsar (Hessels et al. 2006), the shortest orbital period of any known radio pulsar (Camilo et al. 2000), an analysis of the surface mass density of globular clusters (Freire et al. 2001a), and the detection of ionised intracluster gas (Freire et al. 2001b). However, globular cluster surveys are computationally demanding since the survey requires sensitivity to millisecond pulsars. Such a survey therefore requires fast sample times and a large number of frequency channels.

As ASKAP will be sited in the southern hemisphere it will provide the opportunity to carry out new surveys of the Magellanic Clouds. Before the advent of the SKA, the Magellanic systems offer our only hope of detecting pulsars in galaxies other than our own. There are 20 pulsars currently known in the LMC and SMC combined (Manchester et al. 2006). The FoV of ASKAP is ideally suited to targetting the clouds in a single pointing. A very deep search of the clouds could therefore be envisaged. The DM distribution of pulsars in the clouds leads to an evaluation of the ionized medium therein, and the pulsar population as a whole probes high mass star formation and destruction in an environment very different from that of the Milky Way.

\subsection{Timing Pulsars}

The assumption that a pulsar is a regular rotator following a predictable slow-down model forms the basis of a powerful technique for finding its rotational, positional and orbital properties. Pulsars are commonly divided into three categories (see Figure 40):

- The millisecond pulsars are fast spinning and have small spin-down rates. These pulsars are generally extremely stable and their signals can be used for testing relativistic orbital mechanics, measuring stellar masses, probing structures in the interstellar medium and even for detecting a stochastic background of gravitational waves.

- The normal pulsars have much stronger magnetic fields than the millisecond pulsars, are younger and often exhibit large and often unpredictable variations in their 
periods. Timing analyses of young pulsars have allowed their astrometric, binary and spin-down parameters to be determined, leading to improved formation and evolution models. Sudden increases in the pulsar's spin rate (known as 'glitches') are the only probe of the superfluid interior structure of neutron stars, and lead to fundamental insights into the behaviour of matter under extreme densities and pressures.

- The unusual pulsars. These include the recently discovered rotating radio transient objects (RRATs) in which only a few individual pulses of radiation are detected over observations spanning $\sim 30$ minutes (McLaughlin et al. 2006). A new class of pulsar has also recently been identified in which the pulse shape suddenly changes, or in extreme cases, the pulsar turns off for long quasi-periodic stretches of time (Kramer et al. 2006). Long-term timing analyses help to elucidate such phenomena. Almost since the discovery of pulsars it has been known that the pulse emission can sometimes switch off for short periods of time. Even with more than thirty years of pulsar observations the physical mechanism causing these 'switching-off' phenomena is still not understood.

There are numerous pulsar timing programs currently being carried out worldwide. The requirements for the timing of normal pulsars are not difficult to achieve and ASKAP will be competitive with other major observatories. ASKAP has three main advantages: 1) it is sited in the southern hemisphere providing the opportunity to observe pulsars that only one major pulsar-timing telescope (Parkes) can currently see, 2) it is located in the world's most radio-quiet site, which greatly benefits pulsar observations that are very sensitive to radio frequency interference, and 3) the large FoV means that multiple pulsars can be observed simultaneously. The requirements for millisecond pulsar timing are much more stringent. It is likely that the restricted frequency coverage in the current design of ASKAP will limit the precision with which pulsars can be timed. However, as described below, ASKAP will be able to provide a useful timing data-set for a few millisecond pulsars and will be used as a test-bed for high precision pulsar timing in the SKA era.

\subsubsection{Normal Pulsars}

Approximately 1600 normal pulsars are currently known. It is likely that this number will significantly increase with the new surveys being undertaken world-wide (and with ASKAP). In order to achieve maximum science from these discoveries it is necessary to observe repeatedly each pulsar over a data span of approximately one year. After this initial observing period, the basic astrometric and rotational parameters are known and a decision can be made whether the pulsar is of sufficient interest for continued observations.

ASKAP will be able to monitor all the pulsars discovered during the surveys described above. This would consist of approximately fortnightly monitoring over 12 months with observations typically lasting between $\sim 10 \mathrm{~min}$ and $1 \mathrm{hr}$. This will allow the pulsar parameters to be determined and the existence of any interesting binary systems, glitching pulsars or other unusual phenomena to be identified. For instance, we plot the timing residuals for PSR B1828-11 in Figure 41. The sinusoidal signal seen in the timing residuals for this pulsar is explained as the neutron star undergoing free precession (Stairs et al. 2000). As ASKAP can also be used as an imaging interferometer it will be possible to obtain accurate pulsar positions reasonably quickly. This 
$1828-11(\mathrm{rms}=12854.000$ us $)$ post - fit

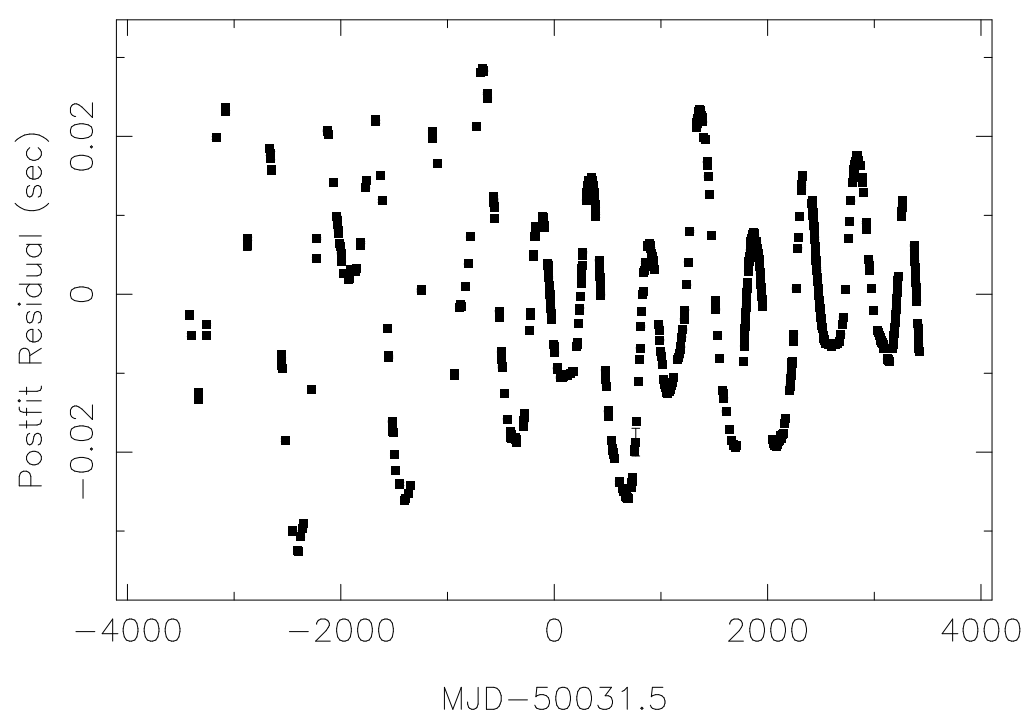

Fig. 41 The timing residuals for PSR B1828-11 obtained using the Lovell telescope. The sinusoidal signal (along with the simultaneous variation in profile shape) is explained as the neutron star undergoing free precession. See Stairs et al. (2000) for further description.

in turn will allow quicker determination of the other pulsar parameters than would be possible without knowledge of the pulsar's position.

Long-term monitoring of a subset of "interesting" pulsars will be desirable. Observations made every few weeks over the period of a few years would allow, for example, the determination of proper motions, orbital effects and the careful study of glitch events.

The timing capabilities of ASKAP will provide the essential ephemerides for southern hemisphere pulsars for any high-energy satellite mission in operation at the time. New discoveries from these missions can then be rapidly timed since there can be multiple beams within the large FoV of ASKAP. Large telescopes, such as Parkes, are currently observing many normal pulsars. ASKAP would be able to reduce the observing load at such telescopes by monitoring multiple pulsars simultaneously.

\subsubsection{Requirements}

The requirements for the timing of normal pulsars are not stringent. However, it does require that ASKAP produce at least one phased-array beam. It is likely that the large FoV will provide the opportunity to observe multiple pulsars simultaneously, thereby reducing the total time required to observe all the requested pulsars. Therefore the creation of multiple phased-array beams within the FoV would be useful, especially if they can be formed independently (simultaneously) with the standard imaging correlator operations. This could allow pulsar timing to be carried out in "piggy-back" mode with other observations. A moderately powerful pulsar backend system is required for each phased array beam and observing frequencies close to $700 \mathrm{MHz}$ would be ideal. 


\subsubsection{Millisecond Pulsars}

One of the most exciting modern-day applications of millisecond pulsar timing is to combine data from multiple pulsars to form a global timing array (Foster \& Backer 1990; Hobbs 2005). The aims of such a project are manifold with the main goal of making a detection of the gravitational wave (GW) background at nano-Hertz frequencies. In brief, GW backgrounds are predicted to occur (e.g. Maggiore 2000) due to cosmological processes (e.g. inflation, cosmic strings or phase transitions), or astrophysical processes (e.g. coalescing massive black hole binary systems resulting from the mergers of their host galaxies). The background is detected by looking for correlations between the timing residuals of pulsars that have a wide range of angular separations (Jenet et al. 2005).

High-precision millisecond-pulsar timing will also continue to undertake sensitive observations of relativistic effects in double-neutron-star systems which lead directly to stringent tests of relativistic gravity. ASKAP will have uniquely sensitive access to many of the binary pulsar systems discovered in the Parkes multibeam surveys. Masses measured by ASKAP will add to the currently poor statistics of masses in pulsar white-dwarf systems and finally allow a realistic investigation of mass dependencies on orbital period, companion type and evolutionary history. Precise measurement of a very large pulsar mass has potential to constrain the equation of state of dense matter (e.g. Lattimer and Prakash 2004).

The initial design for ASKAP does not provide enough frequency coverage, low enough system temperatures nor a large enough aperture to compete with the precise timing data sets being obtained using the Parkes $64 \mathrm{~m}$ and other telescopes. However, with careful choice of observing systems, it will be possible for ASKAP to provide data on a few pulsars that will be useful for a global timing array project. This will reduce the observing requirements at other telescopes. An alternative, producing even highersensitivity data, is to time millisecond pulsars using ASKAP tied with Parkes. One of the major science drivers for the SKA is to carry out pulsar timing array research. As at least part of the SKA is likely to be built using large numbers of small antennas, it is essential that the techniques required to carry out high precision timing using such antennas have been developed and tested. ASKAP will be the ideal instrument for such studies.

\subsubsection{Requirements}

The requirements for high-precision pulsar timing are manifold. The observations require high polarisation purity $(0.1 \%)$, large bandwidths $(300 \mathrm{MHz}$ at $1400 \mathrm{MHz}$ as a minimum), observing frequencies close to $1.4 \mathrm{GHz}$ with simultaneous observations at both higher and lower frequencies, backends that support on-line pulsar folding (with at least 2048 phase bins and 2048 frequency channels with 8-bit sampling), and online coherent dedispersion systems. For this project there are no requirements for long baselines, nor for a large FoV, because the number of pulsars useful for high precision timing is small. The polarisation purity should be readily achievable as the pulsar will be at the phase centre in a phased-up beam; the polarisation surveys described in Chapter 4 need to achieve this in imaging mode over the entire field of view. 


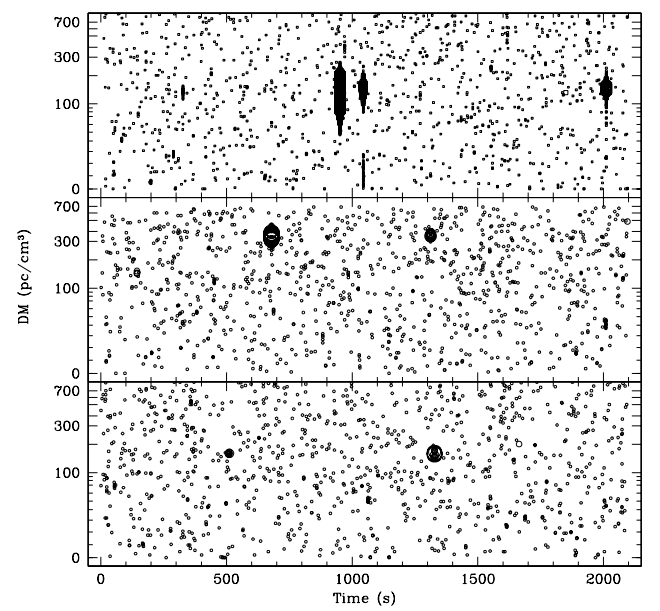

Fig. 42 Discovery plots of rotating radio transient sources from the Parkes Multibeam Pulsar Survey. The concentrated spots indicate detection of single pulses at non-zero dispersion measures. Figure adapted from McLaughlin et al. (2006) and kindly provided by Maura McLaughlin.

\subsubsection{Unusual Pulsars}

The Parkes multibeam pulsar survey has recently discovered rotating transient sources (RRATs) that are thought to be an unusual type of pulsar (see Figure 42). ASKAP provides an excellent instrument for the discovery of more such sources (see Section 7.3). It will also be possible to monitor known RRATs in a similar fashion to the observations of normal pulsars described above. Similarly, it has recently been realised that some pulsars are undetectable for long times, often many weeks, before "switching-on" and behaving as normal pulsars for typically a week or two. These pulsars are difficult to study using existing telescopes and with current observing techniques. The large FoV provided by ASKAP will allow long-term monitoring of these objects (possibly simultaneously with other programs).

\subsection{Fast Transients}

Fast radio transients, those with periods of $1 \mathrm{~s}$ or less, are generally associated with coherent emission processes. Examples of known fast transients include the giant pulse emission from radio pulsars (Johnston \& Romani 2003) and flares on the Sun and other active stars.

Lorimer et al. (2007) discovered a "bright millisecond radio burst of extragalactic origin" by examining archival data obtained in a pulsar search of the Magellanic Clouds (Manchester et al. 2006). The object had a peak flux density of $\sim 30 \mathrm{Jy}$, over 100 times the detection limit. The high dispersion measure for the pulse argues that the object responsible is at $0.1<\mathrm{z}<0.3$ but the large positional error of 10 arcmin precludes the possibility of identifying the host galaxy.. The radiation must be coherent from a 
compact object, and Lorimer et al. note that the only known types of radio sources which could be candidates are RRATs (Section 7.2), or giant pulses from either a millisecond pulsar or a young energetic pulsar. There are arguments making neither possibility likely and Lorimer et al. conclude that a new phenomenon is most likely responsible. They also conclude that there could be over 200 such events detectable per day.

Speculation abounds in the literature as to these other putative sources of fast radio transients. Possibilities include the explosions of primordial black holes (Phinney \& Taylor 1979), and emission from merging neutron stars (Hansen \& Lyutikov 2001) and magnetars (Lyutikov 2003).

Detecting fast transients is difficult, because the volume of data to sift is large, and because the effects of dispersion through the interstellar and galactic media broaden the pulse. A comprehensive review of fast radio transients and search techniques is given in Cordes \& McLaughlin (2003).

ASKAP potentially opens up new parameter space to fast transients because of its large FoV and interference-free environment. A transient search would involve the acquisition, storage and processing of large quantities of data. All these factors scale as the square of the baseline length; it is only feasible to search for fast transients using extremely short baselines (likely less than $300 \mathrm{~m}$ or so). A very compact configuration is therefore desirable, and even then ultra-fast sampling will be challenging.

Another possibility is effectively to discard the interferometric capabilities of ASKAP and to sample and record the outputs of each antenna individually, afterwards summing these outputs incoherently. In this case, one can sample very fast to maintain sensitivity to the most rapid transients over the full FoV, although at the expense of sensitivity to the tune of the square root of the number of antennae.

It seems likely, at least initially, that fast transients are best detected as part of the pulsar survey with $\sim$ ms type sampling rates. Such a survey would certainly detect RRATs, possibly giant pulses, and retains a possibility of detecting unknown once-off events such as exotic mergers. Targeted observations of selected sources at ultra-fast rates may also be possible, e.g. searching for giant pulse emission from the nearest galaxies.

Consideration should also be given to establishing a circular transient buffer of duration a few tens of seconds. This would hold data taken at the fastest possible rate. If any external trigger (e.g. a gamma-ray burst) were to arise in the appropriate part of sky, the buffer could be saved for subsequent analysis.

\subsection{Pulsar VLBI}

Pulsar VLBI using ASKAP is covered in detail in Chapter 6. In brief, a large interferometry system in the southern hemisphere, with the ability to carry out VLBI between the east and west coasts of Australia, will allow many pulsar positions, proper motions and parallaxes to be determined. A single long baseline is adequate for such experiments.

Compared to the northern hemisphere, there are currently few interferometric pulsar parallaxes measured in the south, mostly because they are difficult to obtain using available instrumentation. Currently the baselines (Parkes - ATCA - Mopra - Hobart) are primarily north-south, and Tidbinbilla offers limited availability. ASKAP provides a long east-west baseline, a high-sensitivity station and an immediate improvement in 
$u v$ coverage. The focal plane arrays potentially give observations with multiple beams allowing simultaneous observation of calibrator sources. Even though ASKAP has limited frequency coverage, the specified range is suitable for pulsar VLBI experiments.

\subsection{Expansion of ASKAP}

The expansion option of ASKAP allows for greater collecting area and lower system temperature. The decrease in system temperature is particularly beneficial for the pulsar survey. The survey outlined above could be done in half the time (even without the increase in collecting area), reducing the total data volume and the processing requirements. Pulsar timing will improve, with high signal-to-noise pulse profiles leading to better timing precision. The extended ASKAP could provide high-precision timing data sets of millisecond pulsars comparable with other telescopes, if a wide enough frequency coverage is available. (An ideal system would allow simultaneous observation at three frequencies close to $600 \mathrm{MHz}, 1.4 \mathrm{GHz}$ and $3 \mathrm{GHz}$.)

ASKAP will be a useful instrument for pulsar astronomy in the coming years. However, we emphasise that pulsar science demands a large total aperture, wide frequency coverage, as much central concentration of the aperture as possible, and low system noise. 
8 The Transient and Variable Radio Sky

Lead authors: S. Johnston, I. Stairs

Contributing authors: R. Ekers, C. James, J.-P. Macquart, R. Protheroe, B. Schmidt, M. Walker

8.1 Summary

The good sensitivity and large field-of-view make ASKAP a unique instrument for studying radio transients and variables. We strongly expect that ASKAP will make a unique discovery in the transient parameter space, although it is naturally hard to predict the nature of a new class of transients.

The key to a successful transient instrument is that it have high sensitivity, large field-of-view, good dynamic range and high resolution. ASKAP fulfills these criteria with its ability to achieve sub mJy sensitivity across the entire sky in a single day observing. Nearly all transients arise from point source objects; high resolution is ideal for obtaining accurate positions necessary for follow-up at other wavebands. It is important also that a wide range of timescales from seconds to months are covered by the transient detector. This implies a careful search strategy for uncovering rare objects.

ASKAP will most likely suffer from a surfeit of transient and variable sources. This will pose challenges both for imaging and for determining which sources are most interesting and worthy of follow-up observations on other facilities. Chapter 6 describes some possible mechanisms for VLBI follow-up of transient sources.

\subsection{Introduction}

It has been the general trend in radio astronomy to move from dipole-like antennas towards parabolic dishes which have much larger forward gain at the expense of a much smaller field-of-view (FoV). Consequently, this severely limits the possibility of detecting (random) transient events and the transient sky in the radio is only poorly characterised. At the same time, many classes of objects are known to be variable radio sources including the Sun, the planets, cool stars, stellar binary systems, pulsars, supernovae (SN), gamma-ray bursts (GRBs) and active galactic nuclei (AGN).

Since the 1990s, large-scale blind surveys for transients in the radio band have been performed by a number of groups. Amy et al. (1989) built a transient detector used in parallel with normal observing on the Molonglo Observatory Synthesis Telescope. Although they had good sensitivity and covered a significant fraction of the southern sky in more than $4000 \mathrm{hr}$ observing, they detected only known pulsars. Katz et al. (2003) used detectors in three locations across the USA and observed the entire visible sky over a period of 18 months with a sensitivity of $\sim 27 \mathrm{kJy}$ to transients with periods longer than $0.1 \mathrm{~s}$. The widely separated antenna locations allowed rejection of interference and the only astronomical signals they detected were solar bursts. Matsumura et al. (2007) observed the sky at a declination of 41.5 degrees for 27 continuous days. They detected two transient sources with flux densities in excess of $1 \mathrm{Jy}$ which were only present on a single day. The origin of these transients is unclear. Bower et al. (2007) searched for transient sources in a single VLA field on nearly 1000 occasions 
over the past 22 years. They detected 10 transient sources. Two (possibly four) sources are associated with optical galaxies. The remaining objects have no known counterpart at any other wavelength, nor do they have any persistent radio emission. Their origin remains unknown. Yet another approach is to search archival pulsar data as Lorimer et al. (2007) did; their discovery of a single millisecond pulse of extragalactic origin with the astonishing peak flux density of $30 \mathrm{Jy}$ is described in Section 7.3.

Transients can be conveniently divided into fast (1 second or less) and slow transients; fast transients are dealt with in Chapter 7. Detection of fast transients involves a significant computational load; in contrast, slow transients can be detected directly in the $u v$ plane using the normal data flow through the imaging correlator. In fact the telescope and data acquistion requirements are a subset of those for pulsar detection (see Section 7.2.1). In this chapter we consider the known classes of transients and their likely detection rate by ASKAP. We then speculate on what new classes of transient sources ASKAP might detect in a blind survey.

\subsection{Gamma-Ray Bursts}

GRBs are associated with the most explosive events in the Universe. In the gamma-ray band, they occur approximately twice per day and are visible out to redshifts in excess of 6 . The afterglow of the GRB fades over time and is detected in X-ray, optical and radio bands. In the radio, the power-law decay of the light curve can be modified by the effects of interstellar scintillation causing fast time variability. The high rate of GRBs means it is not practical to follow them all in the radio band using conventional interferometers like the VLA and the ATCA.

There are about 600 GRBs per year and, in the radio, some $25 \%$ are expected to remain visible above $0.1 \mathrm{mJy}$ for $\sim 30$ days (Carilli et al. 2003). This implies some 75 GRBs per year would be visible by ASKAP in the southern hemisphere, and the study of their light curves would provide a large increase in our knowledge in the evolution of these sources at late times.

GRBs are almost certainly beamed in gamma-rays; this implies that we only detect some fraction of the total population. However, it may be possible to detect socalled orphan GRBs without the initial gamma-ray trigger by looking for variable radio sources. Levinson et al. (2002) performed such a search by using data taken with the VLA for the NVSS and FIRST surveys. They detected 9 transient sources present in one data set but not the other. They conclude that several hundred radio afterglows of GRBs should be present in the sky at any one time above a level of $1 \mathrm{mJy}$, similar to predictions made by Totani \& Panaitescu (2002).

\subsection{Supernovae}

ASKAP could provide a census of radio-loud supernovae in the nearby Universe. These sources have variability timescales of days or weeks, and an ASKAP survey which reached $1 \mathrm{mJy}$ over this period of time could probe out to at least $50 \mathrm{Mpc}$ (Gal-Yam et al. 2006) for this type of event. Furthermore, detection in the radio band does not have the biases inherent in optical surveys, such as dust absorption in the host galaxy. 


\subsection{Active Galactic Nuclei}

It has become clear over the last decade that AGN variability falls into two categories. Many, indeed most, AGN are intrinsically variable with slow modulations in flux with timescales of months to years. In contrast, some AGN show deep flux modulations in less than a day. These are now known to be caused by scintillation in the interstellar medium of our Galaxy.

\subsubsection{Intrinsic Variability}

Fiedler et al. (1987a) observed a set of 33 AGN, with flux densities of a few Jy, every day for more than 6 years. All sources were variable over this time span with typically smooth variations in flux with quasi-periods of months to years. It is clear that ASKAP will see this sort of variability over the whole sky as a matter of course and be able to produce light curves for a large number of AGN sources. Indeed, this intrinsic variability may be problematic for calibration as ASKAP will use a global sky model as an initial approximation used to calibrate a particular field. Strong variable sources need to be tracked in order to ensure the viability of the calibration process.

\subsubsection{Intra-Day Variable Sources}

A subset of compact quasars show variability on timescales of less than a day: the intraday variable (IDV) sources (Kedziora-Chudczer et al. 1997). IDVs are of astrophysical interest because the small angular sizes $(<50 \mu$ as) that they must possess in order to exhibit interstellar scintillation require them to possess brightness temperatures near or, in many cases, several orders of magnitude in excess of the $10^{12} \mathrm{~K}$ inverse Compton limit for incoherent synchrotron radiation. The $\mathrm{cm}$-wavelength variations observed in AGN on timescales < 3 days are dominated by interstellar scintillation (ISS), rather than intrinsic variability. Two key observations support this: (a) a delay of 1-8 min in the arrival times of the variations at telescopes on different continents as the scintillation pattern sweeps across the Earth (Dennett-Thorpe \& de Bruyn 2002, Bignall et al. 2006); and (b) annual cycles in the variability due to the (orbital) motion of the Earth relative to the scattering material (e.g. Bignall et al. 2003). The MASIV survey confirmed the dominance of ISS as the mechanism of cm-wavelength IDV by showing a strong correlation between $\mathrm{H} \alpha$ intensity (as measured by the WHAM survey) and IDV variability properties (Lovell et al. 2003; Lovell et al. 2007).

A survey with ASKAP could cover the sky every day to a $5 \sigma$ limit of $2 \mathrm{mJy}$, which would detect variability at the $2 \%$ level for $100 \mathrm{mJy}$ sources and at the $20 \%$ level for $10 \mathrm{mJy}$ sources on a daily basis. This is similar to levels achieved in the (targeted) MASIV survey (Lovell et al. 2003) which found that more than $50 \%$ of the sources in the sample were variable. With 17 sources per square degree above $10 \mathrm{mJy}$ in the sky (Jackson 2005), ASKAP would usefully survey some 350,000 AGN each day. It is useful to ensure that the baselines of the ASKAP configuration be long enough to exceed the confusion limit; however, the stability of the confusing structure in each field from one day to the next would enable variable sources to be identified beyond the confusion limit.

An IDV survey would be most usefully conducted near the upper edge of ASKAP's frequency band. Studies of IDV at $2 \mathrm{GHz}$ show that the variability timescales for most sources are in the range 2-14 days at an anticipated survey frequency of $1.6 \mathrm{GHz}$. 

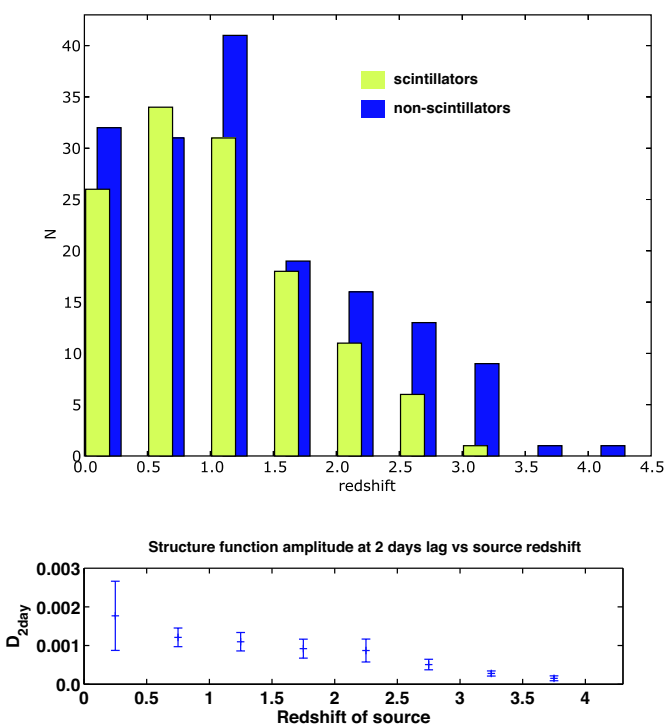

Fig. 43 The redshift dependence of scintillation observed in the MASIV survey. The top panel compares the redshift distributions of sources that showed significant variability and those that did not. The bottom panel shows the drop-off in scintillation amplitude with redshift, using the amplitude of the structure function as a measure of the variability amplitude on timescales up to 2 days.

These timescales are ideally suited to a survey which visits each field once per day. Ultra-fast IDVs can be identified as those whose fluctuations are unresolved on $<1$ day timescales and followed up (at higher frequencies) with the ATCA and the VLA. The dominant mechanism of variability for IDV QSOs at $1.6 \mathrm{GHz}$ is refractive scintillation. The modulation indices of IDVs at $1.6 \mathrm{GHz}$ are expected to be within a factor of two of those at $5 \mathrm{GHz}$, where most previous IDV surveys have been conducted (and where the scintillation amplitude peaks) for two reasons: (i) in the regime of refractive scintillation the expected magnitude of the scintillations scales only weakly, as $\lambda^{-17 / 30}$, and (ii) the sources are expected to be smaller relative to the size of the scattering disk. IDV sources display variations at $5 \mathrm{GHz}$ indicating that their intrinsic angular sizes exceed the Fresnel angular scale and thus that the scintillations are partially quenched. The angular size criterion for unquenched scintillation at $1.6 \mathrm{GHz}$ is $2.5-9$ times less stringent than at $5 \mathrm{GHz}$.

ASKAP stands to answer several fundamental questions about the super-Compton AGN emission exhibited by IDVs.

- IDVs as probes of the intergalactic medium (IGM). IDVs may provide the first direct detection of the ionized baryons in the IGM at $1<z<6$ through angular broadening induced by turbulence in the IGM.

The MASIV survey (Lovell et al. 2007) has shown that few sources above a redshift of 2 are variable (see Figure 43). This suppression of ISS as a function of redshift is most likely caused by scattering in the turbulent ionized IGM, as illustrated in Figure 44. The increased scattering experienced by more distant sources as their radiation propagates through more of the IGM causes them to possess larger apparent angular sizes and thus to show lower levels of ISS as their radiation propagates through our own Galaxy. The abundant amount of turbulent, ionized material in the IGM at these redshifts is likely the result of injection into the IGM from recently formed AGN and remnants of the epoch of reionization. Energy injected into the IGM at large scales is expected to cascade through turbulence until it 
eventually dissipates at some small inner scale. Such a scaling is evident in nearly all astrophysical processes, notably in the interstellar and interplanetary medium (Armstrong et al. 1995, Spangler \& Gwinn 1990).

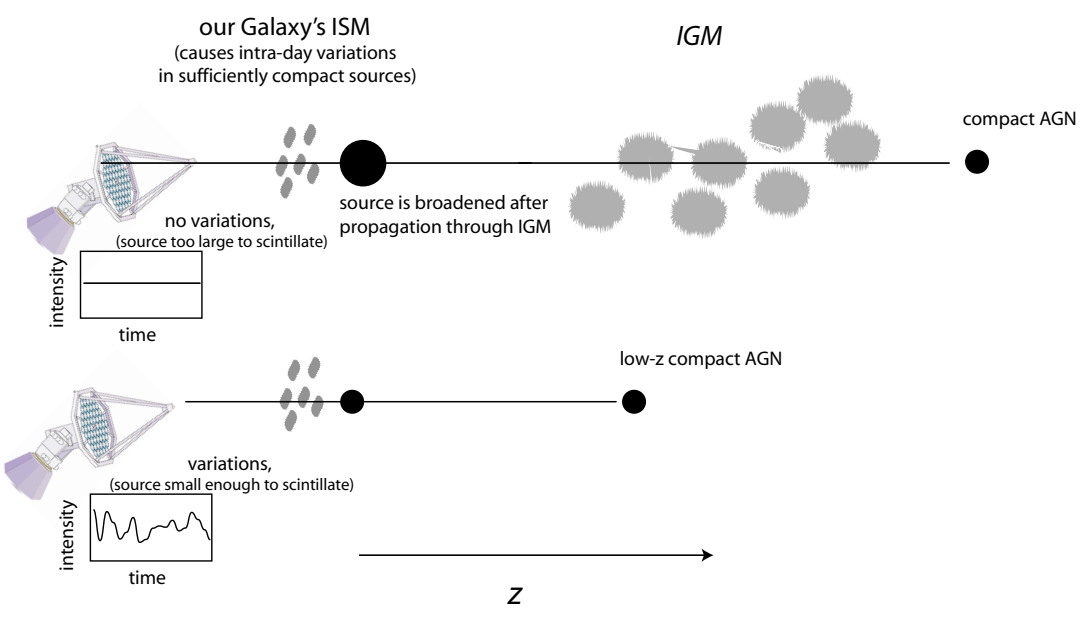

Fig. 44 An illustration of how intergalactic scattering affects the redshift distribution of the variable sources. Intrinsically compact sources at high redshift are subject to more scattering by the intergalactic medium, which broadens their apparent sizes to a point that they no longer appear compact enough to exhibit ISS.

IGM angular broadening is caused by the cumulative effect of all the baryons in the ionized IGM; this is because the process of scatter broadening is caused by phase delays associated with the change in refractive index of the ionized plasma. This is starkly different from the other main probe of the IGM, the Ly- $\alpha$ forest, which is sensitive to a miniscule fraction of the total IGM baryon content; there are estimated to be $\sim 10^{5}$ ionized atoms for every Ly- $\alpha$ absorbing atom detected. This IDV technique is sensitive to turbulence on scales from $10^{8}-10^{11} \mathrm{~m}$, just above the expected turbulence dissipation scale, and can provide a measure of the total energy involved in the turbulent cascade.

The sheer number of sources ASKAP can monitor would enable us to probe in detail the evolution of structure in the ionized IGM as a function of redshift. We could thus probe the evolution of most of the baryonic content of the IGM. However, to make such a contribution, the survey would need to be complemented by optical follow-up to determine the redshifts of the radio sources surveyed.

- IDV is observed to be a highly intermittent phenomenon in most sources. What fraction of IDVs are intermittent, and what is the duty cycle of their IDV? The four-epoch MASIV survey detected variability in $56 \%$ of the 482 sources it surveyed. However, most exhibited IDV only over one or two epochs instead of all four. By continuously observing a large sample over a long period (say 30 days), ASKAPcould comprehensively determine the intermittency properties of IDVs. An intriguing possibility consistent with the MASIV data is that nearly all compact AGN exhibit IDV at one time or another. 
- ASKAP could determine whether IDV intermittency is source- or ISMrelated. The intermittency could be caused either by fluctuations in the turbulent properties of the intervening ISM or by the emergence of bright $\mu$ as structure within the source necessary for the source to exhibit ISS. If the intermittency is ISM-related, the character of any polarization fluctuations associated with the IDV should evolve in the same way as the total intensity fluctuations. However, if the intermittency is source-related, the polarization variations are expected to evolve in a manner different to those in the unpolarized intensity (e.g. due to reconfiguration of magnetic fields internal to the source).

A further complication arises because annual cycles in the variability timescale need to be distinguished from true IDV intermittency. However, these two phenomena can be separated by making a number of observations at identical epochs separated by exactly one year. The variations should be dissimilar between epochs if the source variability is intermittent.

- The brightening and fading of IDV in intermittent sources, which ASKAP could automatically measure in hundreds of thousands of IDVs, would then address the physics that originates the bright emission in the first place. The survey cadence is sufficiently high for studying intermittency in the IDV, which seems to occur on timescales $>14$ days (Kedziora-Chudczer 2006). There is already substantial evidence that IDV intermittency is source-related in several objects (e.g. Macquart \& de Bruyn 2007; Senkbeil et al., in preparation). In this case, the rise and fade times of fast variations in IDVs would address the longevity of $\mu$ as components associated with IDV: the rate at which the AGN supplies energy to power the bright emission to originate the IDV, and the rate at which energy losses cause its eventual decay. These rates can then be compared with specific AGN processes to identify the mechanism associated with the super-Compton radio emission.

\subsection{Extreme Scattering Events}

Extreme Scattering Events (ESEs) are a type of transient in which the flux variations are not intrinsic to the source but are caused by variations in refraction along the lineof-sight (Fiedler et al 1987b; Romani et al. 1987). In other words ESEs are a lensing phenomenon: not gravitational lensing, but refraction of radio waves in ionised gas. It has long been recognised that the lenses which cause ESEs must be Galactic, probably within a few kiloparsecs, but in the 20 years since the phenomenon was discovered there has emerged no satisfactory physical model for the ESE phenomenon. In part this is a reflection of the difficulty of explaining the existing data using established ideas about the interstellar medium. But another reason for the lack of progress is that there has been very little new data on the phenomenon since the early work of Fiedler et al (1987a, 1994; see Figure 45) and the field has stagnated. ASKAP will address this problem in a comprehensive way.

Why study ESEs given that the ESE phenomenon is a bit of an oddity without any obvious connection to other major areas of current astrophysical activity? Analysis of existing data suggests (Walker 2007) that ESEs are caused by dense gas clouds which are unlike any other catalogued component of the interstellar medium. The data suggest that the individual lenses are spherical, and must therefore be associated with neutral, self-gravitating gas clouds, and that they are present in vast numbers in the Galaxy - probably dominating its total mass. In short, one possible interpretation is that the 


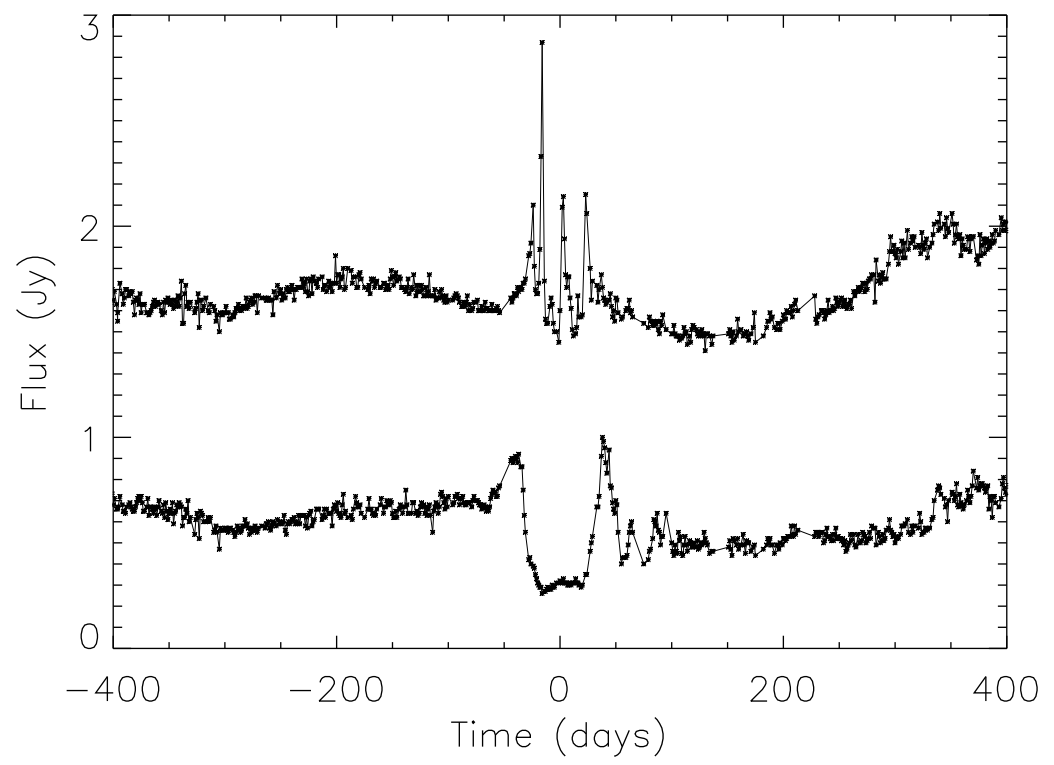

Fig. 45 The Extreme Scattering Event in Q0954+658, at $8.1 \mathrm{GHz}$ (top curve) and $2.7 \mathrm{GHz}$ (lower curve), from Fiedler et al (1987b). An offset of +1 Jy has been added to the highfrequency data for clarity. The mid-point (symmetry point) of the event at Time $=0$ corresponds to MJD44650.00. These data were obtained from http://ese.nrl.navy.mil/.

ESE data appear to be revealing the Galaxy's Dark Matter. Conventional wisdom has it that the Dark Matter is composed predominantly of weakly interacting elementary particles, not baryons, so the ESE data offer a direct challenge to one of the prevailing ideas in cosmology.

Clearly further study of ESEs is warranted. ASKAP is a superb instrument for this purpose, considering the possibilities for monitoring large numbers of compact radio sources. For a nominal FoV of $30 \mathrm{deg}^{2}$, with a $20 \mathrm{sec}$ integration and $10 \mathrm{sec}$ of overhead (slewing and settling time), an area of $15,000 \mathrm{deg}^{2}$ can be surveyed in approximately 4 hours, so it is feasible to do this every day. In a $20 \mathrm{sec}$ integration the noise level in a continuum image (300 MHz bandwidth) should be roughly $0.7 \mathrm{mJy}$ at the upper end of the frequency range for ASKAP (around $1.8 \mathrm{GHz}$ ). A $50 \%$ flux change can therefore be detected as significant in sources brighter than about $10 \mathrm{mJy}$, and to this depth there should be $2.2 \times 10^{5}$ radio sources in the survey area (Wall 1994). Roughly $20 \%$ of these sources are expected to be "compact" based on the models constructed by Jackson \& Wall (1999); here we take "compact" to mean that the source is small enough in angular dimension to be able to exhibit an ESE, so that there are $\sim 45000$ sources in the survey area which have the potential to exhibit an ESE. (Clearly this is not the meaning originally intended by Jackson \& Wall (1999), but we lack the information necessary to make a better estimate ourselves.)

The existing data (Fiedler et al. 1994) have a total coverage of approximately 600 source-years; a four-year ESE survey with ASKAP will improve on this by a factor of roughly 300 . There will be a number of benefits which follow immediately from the 
increased coverage, namely: better definition of the light-curves of ESEs; information on the number and distribution of lenses in the Galaxy; and measures of the clustering of the lenses. What is even more important, however, is the possibility of discovering ESEs in real time, while the events are in progress, and studying them in detail with instruments other than ASKAP. Events like those seen by Fiedler et al. (1994) in Q0954+658 and Q1749+096 should be seen at the rate of roughly 60 per year, with 10 in progress at any given moment. We could study individual events with full polarisation information, good temporal sampling and good frequency coverage; and all this at high signal-to-noise ratio. Such data would be of tremendous value. We would do better still if we could also obtain high resolution (VLBI) images of the evolving source structure during the events, showing how the lenses distort the appearance of the source at various times. Multiple imaging is expected and the various images are expected to rotate on the sky, change in brightness, appear and disappear over the course of an event. At present it is not clear whether it will be possible to resolve the image distortions caused by an ESE, and even attempting the observations will be difficult because the VLBI network has to be scheduled in real time, but if these effects can be measured then the data will be place strong constraints on the electron column-density profile of the lenses, their distances and transverse velocities. Finally there are some key measurements which could be made at higher frequencies - i.e. the optical to X-ray band - which should directly reveal the dense neutral gas clouds responsible for ESEs via scattering, absorption and perhaps refraction (Draine 1998).

\subsection{Stars}

\subsubsection{Flare Stars}

A surprisingly large fraction of cool stars, with spectral types of $\mathrm{M}$ and later, are known to flare in the radio band (Berger 2006). These flares can be used to measure the magnetic field strengths of the stars, and indeed Berger (2006) argues that radio observations are the best way to obtain these values. Burgasser \& Putman (2005) show an example of a spectacular flare from an M dwarf which peaks at $30 \mathrm{mJy}$ and lasts for a few minutes. The numbers of such events potentially detectable by ASKAP is unclear, but one could imagine taking a census of such stars in the local (10 pc) neighbourhood.

\subsubsection{Cataclysmic Variables}

Cataclysmic variables (CVs) are binary stars consisting of a white dwarf and a red dwarf in orbit about each other with a period of a few hours. The systems are generally divided into two classes depending on the magnetic field strength of the white dwarf. $\mathrm{CVs}$ are variable over all wavelengths and a wide variety of timescales. However, only the nearby magnetic CVs are persistent (weak) radio sources. Novae from CVs are occasionally seen in the radio band with flux densities in excess of $10 \mathrm{mJy}$ (Dulk et al. 1983). Examination of overlapping fields in the VLA FIRST survey for variable radio sources, and subsequent optical follow-up, led Bond et al. (2002) to claim the first radio selected CV. With a total galactic population in excess of $10^{6}$, any deep radio survey for variability is certain to detect many radio flares from these objects. 
Compact stellar objects such as neutron stars or black holes in orbit with periods of a few days about a normal star can accrete material from their binary companion. This accretion produces X-ray emission and the systems are generally known as X-ray binaries. The systems give off relativistic jets and the jet emission is detectable in the radio band. Several hundred of these X-ray systems are known out of a likely Galactic population of tens of thousands.

There is a rich radio phenomenology associated with these objects, both in the quiescent and outburst states (see e.g. Fender 2004). In a small handful of objects, the jet is seen expanding at superluminal speeds into the interstellar medium. These observations give insight into the disk/jet interaction and the formation of accretion disks in general. There are obvious links, both observational and theoretical, to the AGN disk/jet structures seen in the distant Universe. For example, there appears to be a scaling between X-ray and radio power in black holes of all sizes (Merloni et al. 2003).

Currently a few tens of objects have significant radio outbursts lasting ten days which repeat on a timescale of a year or so. These sources tend to be bright and easy to monitor. There must surely be an underlying, dimmer population of these objects about which we have little information. A regular sky survey, such as ASKAP will do, should provide more information on these objects, especially when coupled with X-ray observations.

\subsection{Lunar Cherenkov to Detect UHE Particles}

The ability to detect transient radio emission suggests one particular novel proposal which would open a new field of particle physics to radio astronomy. ASKAP is ideally suited, but the observing requirements are severe, as we describe.

The origin of the most energetic particles observed in nature, the ultra high energy (UHE) cosmic rays, which have energies extending up to at least $2 \times 10^{20} \mathrm{eV}$, is currently unknown. Finding the origin of these particles will have important astrophysical implications as they may be accelerated in active galaxies or radio galaxies, and could be directly or indirectly responsible for much of the non-thermal emission in these objects. One promising non-acceleration scenario is the generation of UHE particles due to the decay of super-massive Dark Matter particles which would form Cold Dark Matter halos, with the highest concentration near centres of galaxies. In this scenario we would expect a strong UHE neutrino flux from the Galactic Centre region.

A key to untangling the origin of the UHE cosmic rays will be observations of UHE neutrinos. Despite the fact that for extragalactic UHE cosmic rays almost all spectral information above the GZK cut-off is lost, significant information on the cosmic ray spectrum at the sources is preserved in the spectrum of neutrinos (Protheroe \& Clay 2004); of course the neutrinos are not deflected by magnetic fields and so should point back to where they were produced. The Moon can be used as a large-volume neutrino detector, adopting a powerful method first proposed by Dagkesamanskii \& Zheleznykh (1989) and first applied by Hankins et al. (1996) using the Parkes radio telescope. Known as the Askar'yan effect (after Askar'yan (1962) who first predicted coherent Cherenkov emission in dielectric media at radio frequencies), coherent radio 
Cherenkov emission from neutrino-induced electromagnetic cascades in the lunar regolith (the $\sim 10 \mathrm{~m}$ deep sandy lunar surface layer) can be observed with ground-based radio telescopes.

Cherenkov radio pulses are much shorter (sub-nanosecond duration) than any signals normally encountered in radio astronomy, so that special signal processing techniques are required. The spectrum of coherent Cherenkov emission rises approximately linearly with frequency until a peak value is reached, after which de-coherence and/or attenuation in the regolith result in a relatively sharp cut-off at about $5 \mathrm{GHz}$. Far from the Cherenkov angle, and deeper in the regolith, the peak moves down to about $100 \mathrm{MHz}$.

ASKAP is an array of small dishes representing the optimum way to do this experiment. The dishes are small enough to see the entirety of the moon at $1.4 \mathrm{GHz}$ and the array and site provide essential discrimination against RFI. To gain the advantage of the large number of small dishes, signals have to be combined and analysed with nanosecond timing accuracy. This can be done by using the phased-array beam-forming hardware. The extremely short Cherenkov pulses suffer dispersion in the ionosphere which must be corrected in real time at the output of the phased array beams to maximise the signal-to-noise ratio. This will be similar to the pulsar back-end requirements. We need enough phased array beams to cover all the limb of the moon and this will depend on frequency and resolution. A $5 \mathrm{~km}$ array at $1.5 \mathrm{GHz}$ would already need 250 beams which is impractical. For a reasonable system of say 25 beams we will only be able to use the inner $0.5 \mathrm{~km}$, and a high central concentration or an ultra-compact configuration is a huge advantage. Note that the compact core with a large fraction of the collecting area is needed to optimise the signal-to-noise for the event trigger, but once triggered, buffered data from the longer baselines can be used for position determination. Although there has been no explicit discussion of the ASKAP phased-array beam-forming machinery needed for VLBI, pulsars or neutrino pulses, it is interesting to note that it may be possible to reconfigure the FPA beam-forming hardware for use as the antenna-array beam-formers when large fields of view are not required.

\subsubsection{Comparison with Other Facilities}

In the UHE neutrino energy range applicable to this experiment, $10^{18}-10^{23} \mathrm{eV}$, the ANITA balloon-borne experiment (Barwick et al. 2006), which observes the Antarctic ice shield looking for coherent radio Cherenkov emission in ice from cascades initiated by UHE neutrinos, has the greatest sensitivity to UHE neutrinos. Because the Antarctic ice shield is at the pole, ANITA is only sensitive to UHE neutrinos from the equatorial plane region from declinations approximately $|\delta|<15^{\circ}$. Hence the lunar Cherenkov technique will play a very important and complementary role in UHE neutrino and cosmic ray astronomy at $|\delta|>15^{\circ}$, inaccessible to ANITA; see Figure 46. This includes the nearby blazar Mark 421 and nearby FRI radio galaxy Cen A, as well as the Galactic Centre - all likely sources of UHE cosmic rays and neutrinos but not visible to ANITA.

\subsubsection{Observing Considerations}

The dependence of the peak frequency on shower geometry makes the choice of an optimum observation frequency non-trivial. Higher frequencies have a narrower acceptance angle and this reduces the effective aperture. But lower frequencies have reduced sensitivity, increasing the particle energy threshold to above $10^{21} \mathrm{eV}$. Since the flat-spectrum 


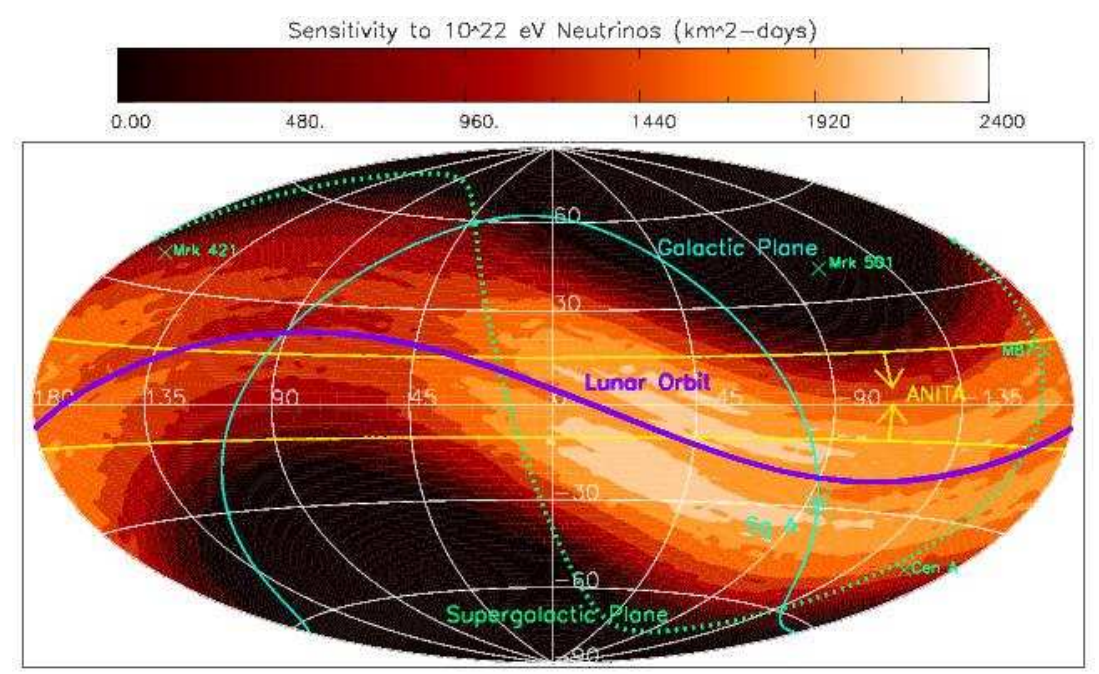

Fig. 46 Likely sensitivity ( $\mathrm{km}^{2}$-days) to $10^{22} \mathrm{eV}$ neutrinos of ASKAP in celestial coordinates for one month of observations ( $40 \%$ duty cycle). A frequency band of $0.7-1 \mathrm{GHz}$ is assumed. The sky coverage is much broader than for the ANITA Antarctic ice balloon experiment (Miocinovic et al. 2005; reproduced with the kind permission of the authors).

Z-burst models of cosmic-ray production have been ruled out by the ANITA-lite and FORTE limits, we place greater emphasis on observing below $10^{21} \mathrm{eV}$ and hence the higher frequencies. By observing at higher frequencies the de-dispersion needed to correct for the ionosphere is smaller and easier to correct, and the increased sensitivity to shower geometry provides more information on the energy and arrival direction of the primary particle.

There are very strong and complementary arguments in favour of observing at low frequencies (Scholten 2006). This applies especially to cosmic ray primaries for which only radiation far from the Cherenkov angle is likely to escape the regolith. (Cosmic rays will interact immediately so that the bulk of their radiation will be directed into the Moon, while neutrinos can penetrate enough of the limb to produce showers parallel to the surface.) The low frequency observations proposed for LOFAR and also possible with the MWA provide complementary information on UHF particles.

The effective aperture of the full SKA as a function of neutrino energy is comparable to ANITA and reaches the flux level at which GZK neutrinos must be seen. Sensitivities have been modelled for ASKAP, and compared with the published apertures for GLUE and ANITA. The neutrino flux sensitivities for a 40-day exposure with ASKAP at $10^{12} \mathrm{GeV}$ is 100 times the sensitivity of GLUE or the Parkes telescope, 5 times better than ATCA, but still 20 times less than the proposed ANITA or SKA sensitivity. LOFAR (or MWA) has no sensitivity at $10^{21} \mathrm{eV}$ but exceeds the sensitivity of all other experiments at energies greater than $10^{22} \mathrm{eV}$.

The key observing requirements are as follows:

- The pulse-detection equipment will require a non-standard back-end, with very fast special-purpose signal processing.

- Full polarisation is needed. 
- The largest possible bandwidth is essential.

- The $0.8-1.7 \mathrm{GHz}$ frequency range is optimum for neutrino energy sensitivity and for neutrino vs cosmic-ray discrimination.

- Extension of the upper frequency of ASKAP to $3 \mathrm{GHz}$ would give more information on particle energy and direction and would become very important when UHE neutrinos are detected.

\subsection{New Classes of Transients}

The most interesting transient sources detected with ASKAP will undoubtedly be objects which we currently know nothing about. One of the advantages of an all-sky survey is that it is tailor-made for detecting the unknown. It has become clear recently that the radio sky contains many transient objects, the indentification of which remains mysterious. For example, Hyman et al. (2005) discovered a bursting transient towards the Galactic Centre which lasted for only a few minutes but had a flux density in excess of $2 \mathrm{Jy}$. The bursts repeat at irregular intervals and the identification of this source remains unclear. Subsequently Bower et al. (2007) examined archival VLA data of the same field spanning 22 years with observations approximately once per week. They detected 10 transient sources, at least six of which have no optical counterparts or quiescent radio emission. Bower et al. (2007) consider the classes of known transients and conclude that their sample is unlikely to be drawn from the known population. They estimate that at ASKAP sensitivity, approximately 1 transient source per square degree, with a duration of order a week, will be present at any given time.

At this stage we cannot even surmise what ASKAP will end up detecting, but a regular all-sky survey covering timescales from minutes to months will be a gold mine for radio transient science. 
9 Summary

Section authors: S. Johnston, R. Taylor, J. Wall

Most of the phenomena we observe today, using telescopes to observe across the electromagnetic spectrum, were unknown a few decades ago. Most were discovered by radio astronomers using increasingly powerful instruments; see the list by Wilkinson et al. (2004).

In "Cosmic Discovery", Harwit (1981) addresses the question of what factors lead to new discoveries in astronomy. He argues that a large fraction of the discoveries have been associated with improved coverage of the electromagnetic spectrum or better resolution in the angle, time, or frequency domain. He also notes that astronomical discovery is often closely linked to new technology introduced into the field. Wilkinson et al. (2004) detail just how it is that SKA will extend the multi-dimensional observing space, and they describe considerations in design and operation that are important to enable discovery of the unexpected. These latter precepts are equally vital for the design and operation of ASKAP.

ASKAP is the first of a new generation of radio telescopes using innovative phasedarray feed technology to explore a greatly enlarged survey-area parameter space. This wide field of view - a major step along the SKA path - is essential for many of the science cases presented here. But following Harwit and Wilkinson et al., we can anticipate it leading to the discovery of new, rare and unexpected phenomena. As pointers to this, $\S 2.9$ describes one recent and unexpected discovery, while $\S 8.8$ presents an unusual proposal which would open a new field of particle astrophysics to astronomers. One explores the capabilities of highest frequency resolution; the other the possibilities of using the highest time resolution.

These exciting possibilities spotlight advancing radio astronomy: ASKAP is a key step on the strategic pathway towards the SKA. The goals of ASKAP, simply stated, are to carry out world class, ground-breaking observations, to demonstrate and prototype technologies for the mid-frequency aspect of SKA, and to establish a site for radio astronomy in Western Australia where observations can be carried out free from the harmful effects of radio interference.

This paper has set out the main science themes to be tackled by ASKAP, themes which play into the major issues confronting astrophysics and cosmology today and which naturally parallel those outlined as science drivers for the SKA: the formation, evolution and population of galaxies including our own, understanding the magnetic universe, exploring the poorly-understood transient radio sky, and directly detecting the gravitational waves which must permeate our Universe. 


\section{References}

Afonso, J. et al. 2003, ApJ, 597, 269

Amy, S., Large, M., Vaughan, A. 1989, PASA, 8, 172

Antonucci, R. R. J., Miller, J. S. 1985, ApJ, 297, 621

Appleton, P. N. et al. 2004, ApJS, 154, 147

Archibald, E. N. et al. 2002, MNRAS, 336, 353

Armstrong, J., Rickett, B., Spangler, S. 1995, ApJ, 443, 209

Askar'yan, G. A. 1962, J. Phys. Soc. Japan Suppl, 17, 257

Audit, E., Hennebelle, P. 2005, A\&A, 433, 1

Avila-Reese, V., Vázquez-Semadeni, E. 2001, ApJ, 553, 645

Bartel, N. et al. 2007, ApJ, 668, 924

Barwick, S. et al. 2006, Phys. Rev. Lett., 96, 171101

Basilakos, S. et al. 2007, MNRAS, 378, 301

Baugh, C. M. et al. 2004, NewAR, 48, 1239

Baugh, C. M. et al. 2005, MNRAS, 356, 1191

Baugh, C. M. 2006, Rep. Prog. Phys., 69, 3101

Beck, R. et al. 1996, ARA\&A, 34, 155

Beck, R. 2001, Space Science Reviews, 99, 243

Beck, R., Gaensler, B. M. 2004, NewAR, 48, 1289

Berger, E. 2006, ApJ, 648, 629

Beswick, R. J. et al. 2007, ASPC, 380, 265

Bicknell, G. et al. 2000, ApJ, 540, 678

Bietenholz, M. F., Bartel, N., Rupen, M. P. 2003, ApJ, 597, 374

Bignall, H. et al. 2006, ApJ, 652, 1050

Bignall, H. et al. 2003, ApJ, 585, 653

Binney J. 2004, MNRAS, 347, 1093

Blake, C., Wall, J. 2002a, MNRAS, 329, L37

Blake, C., Wall, J. 2002b, MNRAS, 337, 993

Blake, C., Wall, J. 2002c, Nature, 416, 150

Blanton, E. L. et al. 2004, ApJ, 612, 817

Blasi, P. et al. 1999, ApJ, 514, L79

Blundell, K.M., Rawlings, S., Willott, C.J. 1999, AJ, 117, 677

Bond, H. et al. 2002, PASP, 114, 1359

Boughn, S., Crittenden, R. 2004, Nature, 427, 45

Bower, G. et al. 2007, ApJ, 666, 346

Bower, R. G. et al. 2006, MNRAS, 370, 645

Boyle, B. J. et al. 2007, MNRAS, 376, 1182

Braun, R., Kanekar, N. 2005, A\&A, 436, L53

Braun, R., Thilker, D. A. 2004, A\&A, 417, 421

Brentjens, M. A., de Bruyn, A. G. 2005, A\&A, 441, 1217

Brisken, W. F. et al. 2002, ApJ, 571, 906

Brown, J. C., Taylor, A. R. 2001, ApJ, 563, L31

Brown, J. C. et al. 2007, ApJ, 663, 258

Brown, R. L., Mitchell, K. J. 1983, ApJ, 264, 87

Brown, R. L., Roberts, M. S. 1973, ApJ 184, L7

Brüggen, M., Kaiser, C. R. 2002, Nature, 418, 301

Brüns, C., Kerp, J., Pagels, A. 2001, A\&A, 370, L26

Brüns, C. et al. 2000, A\&A, 357, 120

Brunt, C. M. 2003, ApJ, 583, 280

Burgasser, A., Putman, M. 2005, ApJ, 626, 486

Butcher, H., Oemler, A. Jnr. 1978, ApJ, 219, 18

Camilo, F. et al. 2000, ApJ, 535, 975

Carilli, C. L. et al. 1992, ApJ, 399, L59

Carilli, C. L., Ivison, R., Frail, D. 2003, ApJ, 590, 192

Carilli, C. L., Rawlings, S. 2004, NewAR, 48, 979

Chamaraux, P., Masnou, J.-L. 2004, MNRAS, 347, 541

Chambers, K. et al. 1987, Nature, 329, 604

Chang, T.-C., Refregier, A., Helfand, D. 2004, ApJ, 617, 794

Chengalur, J.N., Kanekar, N. 2000, MNRAS, 318, 303 
Cole S. et al. 2000, MNRAS, 319, 168

Colless, M. et al. 2001, MNRAS, 328, 1039

Condon, J. J. et al. 1998, AJ, 115, 1693

Condon, J. J. 1992, ARA\&A, 30, 575

Cordes, J. M., Lazio, T. J. W. 2002, astro-ph/0207156

Cordes, J. M., McLaughlin, M. 2003, ApJ, 596, 1142

Cowie, L. L. et al. 1996, AJ, 112, 839

Crittenden, R. G., Turok, N. 1996, Phys Rev Lett, 76, 575

Croton, D. J. et al. 2006, MNRAS, 365, 11

Dagkesamanskii, R., Zheleznykh, I. 1989, Sov. Phys. JETP, 50, 233

Darling, J. et al. 2003, AJ, 125, 1177

Darling, J. 2004, ApJ, 612, 58

Darling, J. et al. 2004, ApJ, 613, L101

Davé, R. et al. 1999, ApJ, 511, 521

Davé, R. et al. 2001, ApJ, 552, 473

de Avillez, M., Breitschwerdt, D. 2004, Ap\&SS, 292, 207

De Breuck, C. et al. 2006, MNRAS, 366, 58

Deller, A. et al. 2007a, PASP, 119, 318

Deller, A. et al. 2007b, ATel \#1037

Dennett-Thorpe, J., de Bruyn, G. 2002, Nature, 415, 57

Dickey, J. M. 1997, ApJ, 488, 258

Dickey, J. M., Crovisier, J., Kazes, I. 1981, A\&A, 98, 271

Di Matteo, T., Springel, V., Hernquist, L. 2005, Nature, 433, 604

Dove, J. B., Shull, J. M. 1994, ApJ 423, 196

Draine, B. T. 1998, ApJ, 509, L41

Dressler, A. 1984, ARA\&A, 22, 185

Dulk, G. et al. 1983, ApJ, 273, 249

Dumke, M. et al. 1995, A\&A, 302, 691

Dunlop, J. S., Peacock, J. A. 1990, MNRAS, 247, 19

Elmegreen, B. G. 1991, ApJ, 378, 139

Elmegreen, B. G., Scalo, J. 2004, ARA\&A, 42, 211

Enßlin, T. A. 1999, in Diffuse Thermal and Relativistic Plasma in Galaxy Clusters, 275

Enßlin, T. A., Gopal-Krishna 2001, A\&A, 366, 26

Fabian, A. C. et al. 2005 MNRAS, 360, L20

Faucher-Giguère, C.-A., Kaspi, V. M. 2006, ApJ, 643, 332

Fanaroff, B. L., Riley, J. M. 1974, MNRAS, 167, 31

Fender, R. 2004 NewAR, 48, 1399

Feretti, L. 2002, IAU Symposium, 199, 133

Ferrière, K. 2005, The Magnetized Plasma in Galaxy Evolution, 147

Feulner, G. et al. 2005, ApJ, 633, L9

Fiedler, R. et al. 1987a, ApJSS, 65, 319

Fiedler, R. et al. 1987b, Nature, 326, 675

Fiedler, R. et al. 1994, ApJ, 430, 581

Foster, R. S., Backer, D. C. 1990, ApJ, 361, 300

Freire, P. C. et al. 2001a, MNRAS, 326, 901

Freire, P. C. et al. 2001b, ApJ, 557, L105

Frick, P. et al. 2001, MNRAS, 325, 649

Gaensler, B. M., Beck, R., Feretti, L. 2004, NewAR, 48, 1003

Gal-Yam, A. et al. 2006, ApJ, 639, 331

Gazol-Patiño, A., Passot, T. 1999, ApJ, 518, 748

Geller, R.M. et al. 2000, ApJ, 539, 73

Glazebrook, K. et al. 2007, ASPC, 379, 72

Goldsmith, P. F., Li, D., Krčo, M. 2007, ApJ, 654, 273

Goldsmith, P. F., Li, D. 2005, ApJ, 622, 938

Gomez, P. L. et al. 2003, ApJ, 584, 210

Granato, G. L. et al. 2001, MNRAS, 324, 757

Granato, G. L. et al. 2004, ApJ, 600, 580

Grimes, J. A., Rawlings, S., Willott, C. J. 2004, MNRAS, 349, 503

Gunn, J., Peterson, B. 1965, ApJ, 142, 1633

Gunn, J. E., Gott, J. R. III 1972, ApJ, 176, 1 
Han, J. L., Qiao, G. J. 1994, A\&A, 288, 759

Han, J. L. et al. 2006, ApJ, 642, 868

Hankins, T., Ekers, R., O'Sullivan, J. 1996, MNRAS, 2831027

Hansen, B., Lyutikov, M. 2001, MNRAS, 322, 695

Harwit, M. 1981 "Cosmic Discovery - The Search, Scope \& Heritage of Astronomy", Basic

Books, Inc., New York.

Haverkorn, M. et al. 2006, ApJS, 167, 230

Heiles, C. 1996, ASPC, 97, 457

Heiles, C., Troland, T. H. 2003, ApJS, 145, 329

Heiles, C., Troland, T. H. 2005, ApJ, 624, 773

Hessels, J. W. T. et al. 2006, Science, 311, 1901

Hewish, A. et al. 1968, Nature, 217, 709

Hobbs, G. 2005, PASA, 22, 179

Hobbs, G. et al. 2005, MNRAS, 360, 974

Hopkins, A. et al. 2000, ExA, 10, 419

Horiuchi, S. et al. 2004, ApJ, 616, 110

Hulse, R. A., Taylor, J. H. 1975, ApJ, 195, L51

Hyman, S. et al. 2005, Nature, 434, 50

Indrani, C., Deshpande, A. A. 1999, New Astronomy, 4, 33

Irwin, M. J. et al 2007, ApJ, 656, 13

Jackson, C. A. 2004, NewAR, 48, 1187

Jackson, C. A., Wall, J. V. 1999, MNRAS, 304, 160

Jackson, C. 2005, PASA, 22, 36

Jenet, F. A. et al. 2005, ApJ, 625, L123

Johnston, S., Gray, A. 2006, SKA Memo Series 72, www.skatelescope.org/PDF/memos

Johnston, S., Romani, R. 2003, ApJ, 590, L95

Johnston, S. et al. 2005, MNRAS, 364, 1397

Joncas, G. 1999, in Interstellar Turbulence, Cambridge: CUP, 154

Juneau, S. et al. 2005, ApJ, 619, L135

Kalberla, P. M. W., Kerp, J. 1998, A\&A, 339, 745

Kanekar, N., Athreya, R. M., Chengalur, J. N. 2002, A\&A, 382, 838

Kanekar, N., Briggs, F. H. 2004, NewAR, 48, 1259

Karastergiou, A., Johnston, S. 2006, MNRAS, 365, 353

Kaspi, V. M. et al. 2000, ApJ, 543, 321

Katz, C. et al. 2003, PASP, 115, 675

Kedziora-Chudczer, L. 2006, MNRAS, 369, 449

Kedziora-Chudczer, L. et al. 1997, ApJ, 490, L9

Keller, S. C. et al. 2007, PASA, 24, 1

Kenney, J. D. P., van Gorkom, J. H., Vollmer, B. 2004, AJ, 127, 3361

Keres, D. et al. 2005, MNRAS, 363, 2

Kilborn, V. A. et al. 2005, MNRAS, 356, 77

Klamer, I. J. et al. 2004, ApJ, 612, L97

Klamer, I. J. et al. 2006, MNRAS, 371, 852

Kolatt, T. 1998, ApJ, 495, 564

Kramer, M. et al. 2004, NewAR, 48, 993

Kramer, M. et al. 2006, Science, 312, 549

Kronberg, P. P. 1994, Rep. Prog. Phys., 57, 325

Lah, P. et al. 2007, MNRAS, 376, 1357

Lane, W. M., Briggs, F. H. 2001, ApJ, 561, L27

Larson, R. B. 2003, Rep. Prog. Phys., 66, 1651

Lattimer, J. M., Prakash, M. 2004, Science, 304, 536

Lenc, E., Tingay, S. 2006, AJ, 132, 1333

Levinson, A. et al. 2002, ApJ, 576, 923

Lewis, I. et al. 2002, MNRAS, 334, 673

Li, D., Goldsmith, P. F. 2003, ApJ, 585, 823

Li, Y. et al. 2003, ApJ, 592, 975

Lockman, F. J. 2002, ApJ, 580, L47

Longair, M. S. 1966, MNRAS, 133, 421

Lorimer, D. R. et al. 2007, Science, 318, 777

Lovell, J. et al. 2003, AJ, 126, 1699 
Lovell, J. et al. 2007, ASPC, 365, 279

Lyne, A. et al. 1998, MNRAS, 295, 743

Lyutikov, M. 2003, MNRAS, 346, 540

Macquart, J.-P., de Bruyn, A.G. 2007, MNRAS, 380, L20

Maggiore, M. 2000, Phys. Rep., 331, 283

Manchester, R. N. et al. 2001, MNRAS, 328, 17

Manchester, R. N. et al. 2005, AJ, 129, 1993

Manchester, R. N. et al. 2006, ApJ, 649, 235

Matsumura, N. et al. 2007, AJ, 133, 1441

Mauch, T. et al. 2003, MNRAS, 342, 1117

McCarthy, P. J. et al. 1987, ApJ, 321, L29

McClure-Griffiths, N. M. et al. 2005, ApJS, 158, 178

McLaughlin, M. A. et al. 2006, Nature, 439, 817

Merloni, A., Heinz, S., di Matteo, T. 2003, MNRAS, 345, 1057

Mesa, D. et al. 2002, A\&A, 396, 463

Meyer, M. et al. 2004, MNRAS, 350, 1195

Meyer, M. et al. 2007, ApJ, 654, 702

Minter, A. H., Balser, D. S., Kartaltepe, J. S. 2005, ApJ, 631, 376

Miocinovic, P. et al. 2005, astro-ph/0503304

Miville-Deschenes, M.-A. et al. 2002, A\&A, 381, 209

Monaghan, J. J. 2002, MNRAS, 335, 843

Moore, B. et al. 1996, Nature, 379, 613

Mulchaey, J. S., Zabludoff, A. I. 1998, ApJ, 496, 73

Murgia, M. et al. 2005, in X-ray and Radio Connections, 8.15

Norris, R. P. et al. 2006, AJ, 132, 2409

Norris, R. P. et al. 2007, MNRAS, 378, 1434

Nunes, N. V., Bartel, N. 1998, ASPC, 144, 331

Omar, A., Dwarakanath, K. S. 2005, JApA, 26, 71

Padovani, P. et al. 2007, ASPC, 380, 205

Papadopoulous, P. P. et al. 2008, ApJ, 684, 845

Papovich, C. et al. 2006, ApJ, 640, 92

Peacock, J. A. 1983, MNRAS, 202, 615

Peek, J. E. G. et al. 2007, ApJ, 656, 907

Péroux, C. et al. 2003, MNRAS, 346, 1103

Phillips, C. J. et al. 2007, MNRAS, 380, L11

Phinney, E., Taylor, J. 1979, Nature, 277, 117

Pietrobon, D., Balbi, A., Marinucci, D. 2006, Phys Rev D, 74, 043524

Poggianti, B. M. et al. 2006, ApJ, 642, 188

Prochaska, J. X., Herbert-Fort, S. 2004, PASP, 116, 622

Prochaska, J. X., Herbert-Fort, S., Wolfe, A. M. 2005, ApJ, 635, 123

Protheroe, R. J., Clay, R. W. 2004, PASA, 21, 1

Putman, M. E. et al. 2003, ApJ, 586, 170

Putman, M. E. 2000, PASA, 17, 1

Quilis, V., Bower, R. G., Balogh, M. L. 2001, MNRAS, 328, 1091

Rand, R. J., Lyne, A. G. 1994, MNRAS, 268, 497

Rao, S. M., Turnshek, D. A., Nestor, D. B. 2006, ApJ, 636, 610

Rao, S. M., Briggs F. 1993, ApJ, 419, 515

Rawlings, S., Jarvis, M. J. 2004, MNRAS, 355, L9

Rees, M. J., Ostriker, J. P. 1977, MNRAS, 179, 541

Reynolds, R. J. 1985, ApJ, 294, 256

Robishaw, T., Quataert, E., Heiles, C. 2008, ApJ, 680, 981

Romani, R., Blandford, R. D., Cordes, J. M. 1987, Nature, 328, 324

Ruzmaikin, A. A., Sokolov, D. D., Shukurov, A. M. 1988, ASSL, 133, Magnetic Fields of

Galaxies

Ryan-Weber, E., Webster, R., Staveley-Smith, L. 2003, MNRAS, 343, 1195

Ryan-Weber, E. V. 2006, MNRAS, 367, 1251

Sanders, D. B. et al. 1988, Science, 239, 625

Scalo, J. 1990 in Physical Processes in Fragmentation and Star Formation, ASSL, 162, 151

Scalo, J., Elmegreen, B. G. 2004, ARA\&A, 42, 275

Schilizzi, R. T. et al. 2007, SKA Memo 100, www.skatelescope.org/PDF/memos 
Scholten, O. et al. 2006, Astropart. Phys., 26, 219

Sholomitskii, G. B., Yaskovitch, A. L. 1990, Soviet Astron. Lett, 16, 383

Shukurov, A. 2005, Lecture Notes in Physics, 664, 113

Silk, J., Rees, M. 1998, A\&A, 331, L1

Simpson, C. et al. 2006, MNRAS, 372, 741

Solanes, J. M. et al. 2001, ApJ, 548, 97

Spangler, S., Gwinn, C. 1990, ApJ, 353, 29

Springel, V. et al. 2005, Nature, 435, 629

Springob, C. M., Haynes, M. P., Giovanelli, R. 2005, ApJ, 621, 215

Stairs, I. H., Lyne, A. G., Shemar, S. 2000, Nature, 406, 484

Stanimirovic, S., Heiles, C. 2005, ApJ, 631, 371

Stanimirovic, S., Lazarian, A. 2001, ApJ, 551, L53

Staveley-Smith, L. 2006, ATNF SKA Memo Series \#6,

www.atnf.csiro.au/projects/askap/Memoseries.html

Stevens, J. A. et al. 2005, 360, 610

Stil, J. M., Krause, M., Beck, R., Taylor, A. R. 2008, ApJ, in press

Storrie-Lombardi, L. J., Wolfe, A. M. 2000, ApJ, 543, 552

Subrahmanyan, R. et al. 2006, ApJ, 636, 172

Taylor, A. R. et al. 2003, AJ, 125, 3145

Taylor, A. R. et al. 2006, NAIC Newsletter, 39, 1

Taylor, A. R. et al. 2007, ApJ, 666, 201

Tingay, S. J. et al. 2002, ApJS, 141, 311

Tingay, S. J., Murphy, D. W. 2001, ApJ, 546, 210

Tingay, S. J. et al. 1996, ApJ, 464, 170

Tingay, S. J. et al. 1995, Nature, 374, 141

Totani, T., Panaitescu, A. 2002, ApJ, 576, 120

Treu, T. et al. 2003, ApJ, 591, 53

Tucci, M. et al. 2004, MNRAS, 349, 1267

Tully, B. 1987, ApJ, 321, 280

Tüllmann, R. et al. 2000, A\&A, 364, L36

Uyaniker, B., Kothes, R., Brunt, C. M. 2002, ApJ, 565, 1022

van Breugel, W. et al. 1999, in The Most Distant Radio Galaxies, 49

Verheijen, M. A. W. et al. 2008, ASPC, 1035, 265

van der Marel, R. P., Franx, M. 1993, ApJ, 407, 525

Vranesevic, N. et al. 2004, ApJ, 617, L139

Walker, M. A. 2007, ASPC, 365, 299

Wall, J. V. 1994, Aus. J. Phys., 47, 625

Wall, J. V. et al. 2005, A\&A, 434, 133

Wall, J. V., Pope, A., Scott, D. S. 2008, MNRAS, 383, 435

West, J. L. et al. 2007, ApJ, 656, 914

Whiteoak, J. B., Gardner, F. F. 1968, ApJ, 154, 807

Widrow, L. M. 2002, Rev. Mod. Phys., 74, 775

Wilkinson, P. N. et al. 2004, NewAR, 48, 1551

Willott, C. J. et al. 2001, MNRAS, 322, 536

Windhorst, R. A. 2003, NewAR, 47, 357

Windhorst, R. A. et al. 1990, ASPC, 10, 389

Windhorst, R. A. et al. 1999, ASPC, 193, 55

Wolleben, M. et al. 2006, A\&A, 448, 411

$\mathrm{Xu}, \mathrm{Y}$. et al. 2006, ApJ, 637, 19

York, D. G. et al. 2000, AJ, 120, 1579

Yun, M. S., Ho, P. T., Lo, K. Y. 1994, Nature, 372, 530

Zheng, X. Z. et al. 2007, ApJ, 661, L41

Zwaan, M. et al. 1997, ApJ, 490, 173

Zwaan, M. et al. 2003, AJ, 125, 2842

Zwaan, M. et al. 2005, MNRAS, 359, L30

Zweibel, E. G., Heiles, C. 1997, Nature, 385, 131 